University of Tennessee Health Science Center

UTHSC Digital Commons

\title{
CNS Penetration of Tyrosine Kinase Inhibitors in Mouse Models
}

Mohamed Elmeliegy

University of Tennessee Health Science Center

Follow this and additional works at: https://dc.uthsc.edu/dissertations

Part of the Medical Cell Biology Commons, and the Medical Molecular Biology Commons

\section{Recommended Citation}

Elmeliegy, Mohamed , "CNS Penetration of Tyrosine Kinase Inhibitors in Mouse Models" (2012). Theses and Dissertations (ETD). Paper 74. http://dx.doi.org/10.21007/etd.cghs.2012.0083.

This Dissertation is brought to you for free and open access by the College of Graduate Health Sciences at UTHSC Digital Commons. It has been accepted for inclusion in Theses and Dissertations (ETD) by an authorized administrator of UTHSC Digital Commons. For more information, please contact jwelch30@uthsc.edu. 


\title{
CNS Penetration of Tyrosine Kinase Inhibitors in Mouse Models
}

\begin{abstract}
For the past three decades, advances in the treatment of central nervous system (CNS) tumors such as malignant glioma have only been modest. One particular challenge facing treatment of brain tumors is the delivery of therapeutically effective concentrations of anti-cancer agents to the target site in the brain. The sanctuary of the brain is protected by several barrier systems such as the blood-brain barrier (BBB) and the blood-cerebrospinal fluid barrier (BCSFB). These barriers restrict the passage of anti-cancer drugs into the brain via several protective mechanisms.
\end{abstract}

In the present study, we used cerebral microdialysis sampling, a technique for sampling unbound molecules in brain extracellular fluid (ECF) via semi-permeable probe, to assess the role of murine ATP Binding Cassette (ABC) transporters Bcrp1, P-gp, and Mrp4 in CNS penetration of molecularly targeted agents under investigation for treatment of malignant glioma. We choose the specific inhibitor of epidermal growth factor receptor (EGFR), erlotinib (TarcevaTM), and the specific inhibitor of plateletderived growth factor receptor (PDGFR), crenolanib, as examples of tyrosine kinase inhibitors currently tested for treatment of malignant glioma. Given the poor microdialysis probe recovery of these lipophilic molecules, we enhanced their recovery by including an affinity-based trapping agent, $10 \%$ hydroxypropylbetacyclodextrin (HPBCD), in the perfusate. Using this technique, we studied erlotinib and its major metabolite, OSI-420, penetration in control and transporter-deficient mice. We showed that Bcrp1 is the main efflux transporter preventing erlotinib and OSI-420 penetration in mouse brain. Intracellular accumulation studies confirmed the role of BCRP in erlotinib and OSI-420 transport. We also characterized the role of solute carrier transporters in erlotinib and OSI-420 brain accumulation. Our data show that erlotinib and OSI-420 are substrates for members of the SLC22A family of uptake transporters, OAT3 and OCT2.

We then sought to characterize the disposition of tyrosine kinase inhibitors in malignant glioma using cerebral microdialysis. We decided to use a transgenic mouse model that highly recapitulates several features of the human glioma including tumor histology and genetic profiles. However, the bregma commonly used as a reference point to place microdialysis cannula does not appear on images derived by magnetic resonance imaging (MRI), the imaging method used to identify the size and location of the spontaneously arising tumors. Thus, we realized that a new technique to implant the microdialysis guide cannula would be necessary. Using angiography studies of mouse brain vasculature and T2-weighted $\mathrm{MRI}$, we identified the intersection of the midline suture and the rostral rhinal vein on the mouse brain surface as a reference point for implanting the microdialysis cannula. This point correlated with the intersection between the midline and the olfactory bulb/frontal lobe border visualized on T2- weighted MRI. Our method allowed for accurate placement of microdialysis cannula in tumors developing in several regions of the mouse brain.

While cerebral microdialysis is commonly used to monitor CNS disposition of single anti-cancer drug at a time, the feasibility of simultaneous sampling of multiple anti-cancer agents via cerebral microdialysis has not been reported. However, combining anti-cancer drugs represents a promising strategy for treatment of resistant CNS tumors, as malignant glioma. Given the role played by EGFR and PDGFR in providing multiple inputs for sustaining glioma cell survival and proliferation, combining inhibitors of EGFR (erlotinib) and PDGFR (crenolanib) represents a promising treatment strategy for these tumors. The goal of our last set of studies was to optimize microdialysis conditions to sample crenolanib and erlotinib as single agents or in combination from tumor ECF in a xenograft mouse model of glioma. By including $10 \%$ HPBCD in the perfusate, probe recovery of both erlotinib and crenolanib was significantly increased. To estimate probe recovery we used the zero-flow rate (ZFR) which estimated stock concentrations with $15 \%$ accuracy. The enhanced recovery achieved by including HPBCD coupled with sensitive analytical 
techniques allowed us to determine crenolanib and erlotinib penetration in tumor ECF under steady state conditions. No significant differences were observed in drug penetration between groups treated with single agent or those treated with both drugs.

In conclusion, we developed techniques to improve microdialysis probe recovery of lipophilic agents administered as single agents or in combination. We also developed an MRIguided method to implant microdialysis cannula in a spontaneous glioma murine model. These techniques enhance our ability to perform microdialysis studies on a large spectrum of anticancer agents in clinically relevant murine models. Using the developed techniques, we identified efflux and uptake transporters that regulate erlotinib CNS disposition. We evaluated the extent of erlotinib and crenolanib penetration in malignant glioma models. Our results shed more light on the extent of tumor penetration of two tyrosine kinase inhibitors currently being tested for treatment of malignant glioma.

\section{Document Type}

Dissertation

\section{Degree Name}

Doctor of Philosophy (PhD)

\section{Program}

Biomedical Sciences

\section{Research Advisor}

Clinton Stewart, Pharm.D.

\section{Keywords}

CNS penetration, Crenolanib, Erlotinib, Microdialysis, Tyrosine Kinase Inhibitors

\section{Subject Categories}

Medical Cell Biology | Medical Molecular Biology | Medical Sciences | Medicine and Health Sciences

\section{Comments}

Two year embargo expired May 2014 


\title{
CNS PENETRATION OF TYROSINE KINASE INHIBITORS IN MOUSE MODELS
}

\author{
A Dissertation \\ Presented for \\ The Graduate Studies Council \\ The University of Tennessee \\ Health Science Center \\ In Partial Fulfillment \\ Of the Requirements for the Degree \\ Doctor of Philosophy \\ From The University of Tennessee
}

By

Mohamed Elmeliegy

May 2012 
Chapter 2 (C) 2011 by The American Association for Cancer Research (AACR), Inc. Chapter 3 (C) 2011 by John Wiley \& Sons, Inc.

All other material (C) 2012 by Mohamed Elmeliegy. All rights reserved. 


\section{DEDICATION}

This dissertation is dedicated to my wife, who supported me all the way, and to my parents.

Without their love, I could not have been who I am today. 


\section{ACKNOWLEDGEMENTS}

I would like to take this opportunity to express my sincere appreciation and gratitude to all those who supported and helped me during this journey. It really takes a village to raise a graduate student. First, I would like to thank my advisor, Dr. Clinton Stewart. Four years ago, he graciously welcomed me in his lab and provided me with everything needed for an excellent project. Through all those years, he was a continuous source of motivation for me. He always directed me when I deviated and corrected me when I was wrong. Thanks Dr. Stewart for everything you taught me. I will always cherish what I learned from you.

No words are enough to express my gratitude to Dr. Angel Montero Carcaboso and Dr. Stacy Throm for their mentorship, help, and full support. I have also been incredibly lucky to enjoy the support of members of my graduate committee, Drs. Shannon Matta, John Panetta, Cynthia Wetmore, Suzanne Baker, and Amar Gajjar. I am sincerely indebted to all of them for their support. I also would like to thank the Baker lab for providing the spontaneous glioma model used in this project. In addition, I would like to thank former and present Stewart lab members Laura Miller, Dr. Michael Tagen, Thandranese Owens, Daniel Groepper, Fan Zhang, and Scott Wherry for their help throughout my project.

My gratitude is extended to the head of the graduate student council at St. Jude Children's Research Hospital, Dr. Gerard Zambetti. I would like to thank Dr. Christopher Calabrese, Dr. Ziwei Zhang, Melissa Johnson, and Monique Payton from the small animal imaging core facility at St. Jude. I also would like to thank Dr. Donald Thomason and Dr. James Patrick Ryan at the University of Tennessee Health Science Center for their support. I would also like to acknowledge the Hal and Alma Reagan Fellowship for providing the stipend for my graduate research in the year of 2011-2012.

I want to thank St. Jude Children's Research Hospital for providing an amazing work environment and a noble goal I worked towards achieving. St. Jude's message and legacy are engrafted in my heart and I will carry this message in my heart wherever I go.

Finally, I would like to thank the most important persons in my life, my wife and my parents. Their outpouring love, sacrifice, and genuine belief in me, made my dream come true. Without them, none of this would be remotely possible. To my wife and parents I say, we made it. 


\begin{abstract}
For the past three decades, advances in the treatment of central nervous system (CNS) tumors such as malignant glioma have only been modest. One particular challenge facing treatment of brain tumors is the delivery of therapeutically effective concentrations of anti-cancer agents to the target site in the brain. The sanctuary of the brain is protected by several barrier systems such as the blood-brain barrier (BBB) and the bloodcerebrospinal fluid barrier (BCSFB). These barriers restrict the passage of anti-cancer drugs into the brain via several protective mechanisms.
\end{abstract}

In the present study, we used cerebral microdialysis sampling, a technique for sampling unbound molecules in brain extracellular fluid (ECF) via semi-permeable probe, to assess the role of murine ATP Binding Cassette (ABC) transporters Bcrp1, P-gp, and Mrp4 in CNS penetration of molecularly targeted agents under investigation for treatment of malignant glioma. We choose the specific inhibitor of epidermal growth factor receptor (EGFR), erlotinib (Tarceva ${ }^{\mathrm{TM}}$ ), and the specific inhibitor of plateletderived growth factor receptor (PDGFR), crenolanib, as examples of tyrosine kinase inhibitors currently tested for treatment of malignant glioma. Given the poor microdialysis probe recovery of these lipophilic molecules, we enhanced their recovery by including an affinity-based trapping agent, 10\% hydroxypropyl-betacyclodextrin (HPBCD), in the perfusate. Using this technique, we studied erlotinib and its major metabolite, OSI-420, penetration in control and transporter-deficient mice. We showed that Bcrp1 is the main efflux transporter preventing erlotinib and OSI-420 penetration in mouse brain. Intracellular accumulation studies confirmed the role of BCRP in erlotinib and OSI-420 transport. We also characterized the role of solute carrier transporters in erlotinib and OSI-420 brain accumulation. Our data show that erlotinib and OSI-420 are substrates for members of the SLC22A family of uptake transporters, OAT3 and OCT2.

We then sought to characterize the disposition of tyrosine kinase inhibitors in malignant glioma using cerebral microdialysis. We decided to use a transgenic mouse model that highly recapitulates several features of the human glioma including tumor histology and genetic profiles. However, the bregma commonly used as a reference point to place microdialysis cannula does not appear on images derived by magnetic resonance imaging (MRI), the imaging method used to identify the size and location of the spontaneously arising tumors. Thus, we realized that a new technique to implant the microdialysis guide cannula would be necessary. Using angiography studies of mouse brain vasculature and T2-weighted MRI, we identified the intersection of the midline suture and the rostral rhinal vein on the mouse brain surface as a reference point for implanting the microdialysis cannula. This point correlated with the intersection between the midline and the olfactory bulb/frontal lobe border visualized on T2-weighted MRI. Our method allowed for accurate placement of microdialysis cannula in tumors developing in several regions of the mouse brain.

While cerebral microdialysis is commonly used to monitor CNS disposition of single anti-cancer drug at a time, the feasibility of simultaneous sampling of multiple 
anti-cancer agents via cerebral microdialysis has not been reported. However, combining anti-cancer drugs represents a promising strategy for treatment of resistant CNS tumors, as malignant glioma. Given the role played by EGFR and PDGFR in providing multiple inputs for sustaining glioma cell survival and proliferation, combining inhibitors of EGFR (erlotinib) and PDGFR (crenolanib) represents a promising treatment strategy for these tumors. The goal of our last set of studies was to optimize microdialysis conditions to sample crenolanib and erlotinib as single agents or in combination from tumor ECF in a xenograft mouse model of glioma. By including 10\% HPBCD in the perfusate, probe recovery of both erlotinib and crenolanib was significantly increased. To estimate probe recovery we used the zero-flow rate (ZFR) which estimated stock concentrations with $15 \%$ accuracy. The enhanced recovery achieved by including HPBCD coupled with sensitive analytical techniques allowed us to determine crenolanib and erlotinib penetration in tumor ECF under steady state conditions. No significant differences were observed in drug penetration between groups treated with single agent or those treated with both drugs.

In conclusion, we developed techniques to improve microdialysis probe recovery of lipophilic agents administered as single agents or in combination. We also developed an MRI-guided method to implant microdialysis cannula in a spontaneous glioma murine model. These techniques enhance our ability to perform microdialysis studies on a large spectrum of anti-cancer agents in clinically relevant murine models. Using the developed techniques, we identified efflux and uptake transporters that regulate erlotinib CNS disposition. We evaluated the extent of erlotinib and crenolanib penetration in malignant glioma models. Our results shed more light on the extent of tumor penetration of two tyrosine kinase inhibitors currently being tested for treatment of malignant glioma. 


\section{TABLE OF CONTENTS}

CHAPTER 1. INTRODUCTION ...................................................................................

1.1. INTRODUCTION OF MALIGNANT GLIOMA …............................................1

1.1.1. Incidence of malignant glioma in adult and pediatric populations .................1

1.1.2. Pathological features of malignant glioma...................................................

1.1.3. Genetic and molecular alterations characteristic of malignant glioma ...........2

1.1.4. Current approaches for treatment of malignant glioma ..................................

1.1.5. Emerging treatments for malignant glioma ……….....................................6

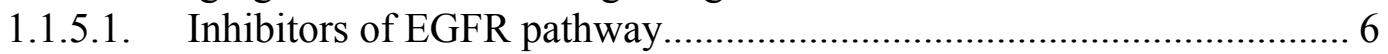

1.1.5.2. Inhibitors of PDGFR pathway ……………....................................... 7

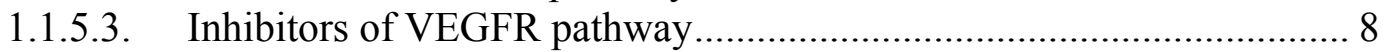

1.1.6. Challenges facing molecularly targeted therapies in malignant glioma ........9

1.2. BARRIERS AGAINST ANTI-CANCER DRUG CNS PENETRATION ...........10

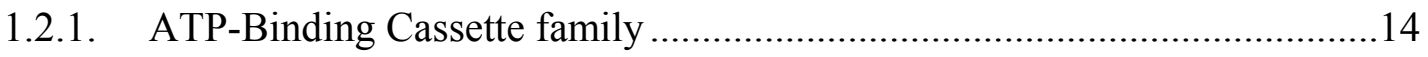

1.2.1.1. P-glycoprotein (P-gp/ABCB1) ...................................................... 14

1.2.1.2. Multi-drug resistance associated proteins (MRPs/ABCC) ................... 16

1.2.1.3. Breast cancer resistance protein (BCRP/ABCG2) ............................. 16

1.2.1.4. Pharmacological inhibition of efflux transporters ................................ 17

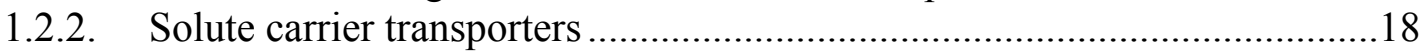

1.2.2.1. Organic anion transporter-3 (OAT3) ……....................................... 18

1.2.2.2. Organic cation transporter-2 (OCT2) ............................................... 18

1.2.2.3. Organic anion transporter polypeptide 1A2 (OATP1A2) .................. 19

1.3. ANTI-CANCER DRUG PENETRATION IN HIGH-GRADE GLIOMAS .......19

1.3.1. The blood-tumor barrier in malignant glioma ..........................................19

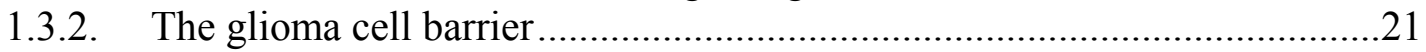

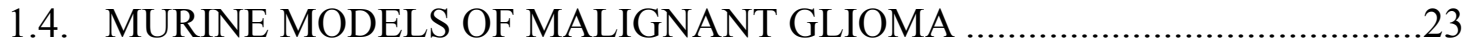

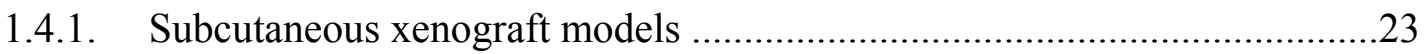

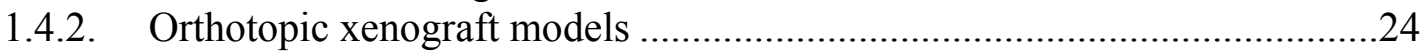

1.4.3. Genetically engineered mouse models.......................................................25

1.5. APPROACHES TO EVALUATE ANTI-CANCER DRUG PENETRATION

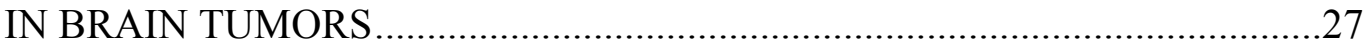

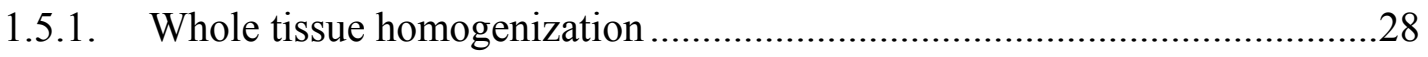

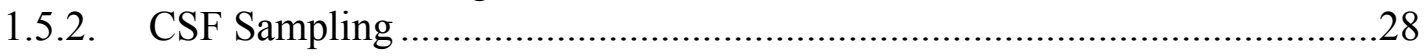

1.5.3. Cerebral microdialysis .....................................................................29

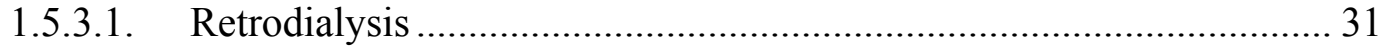

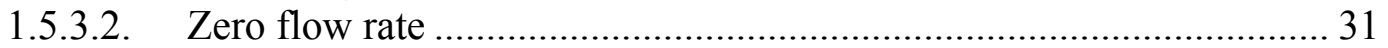

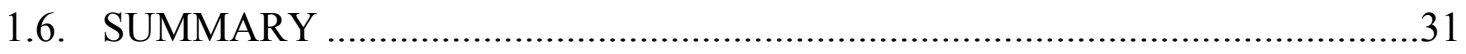

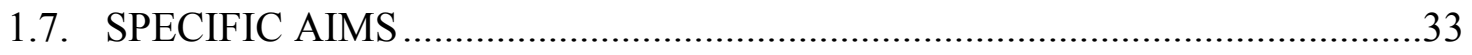

CHAPTER 2. ROLE OF ATP-BINDING CASSETTE AND SOLUTE CARRIER TRANSPORTERS IN ERLOTINIB CNS PENETRATION AND INTRACELLULAR ACCUMULATION* ..........................................................34

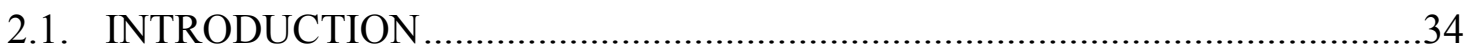

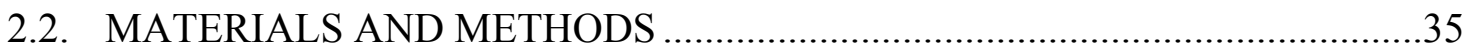




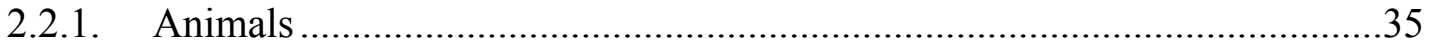

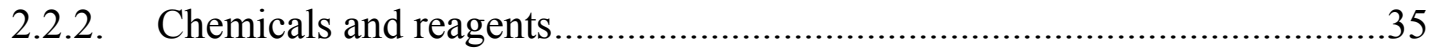

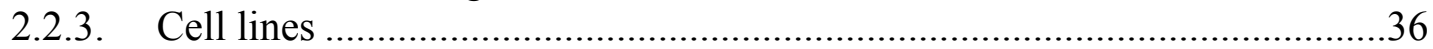

2.2.4. Determination of erlotinib and OSI-420 protein binding in mouse

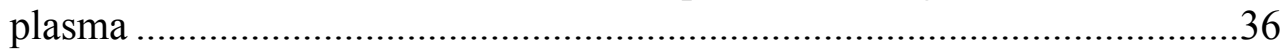

2.2.5. Erlotinib and OSI-420 plasma pharmacokinetics ........................................36

2.2.6. Development of pharmacokinetic limited sampling models for erlotinib

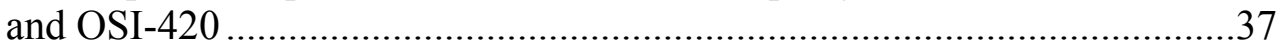

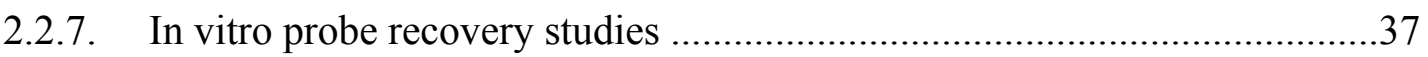

2.2.8. Erlotinib brain microdialysis studies …………........................................38

2.2.9. In vivo microdialysis probe recovery using zero flow rate (ZFR) method..38

2.2.10. Pharmacokinetic analysis of microdialysis studies.....................................39

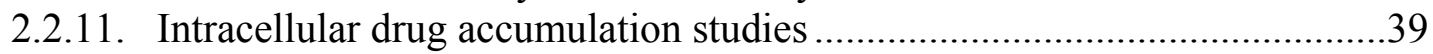

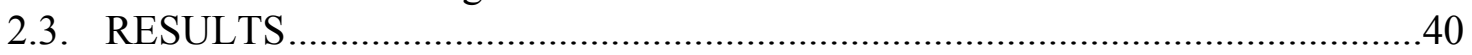

2.3.1. Erlotinib and OSI-420 protein binding in mouse plasma …….....................40

2.3.2. Limited sampling models for erlotinib and OSI-420 .................................40

2.3.3. In vitro microdialysis studies ................................................................47

2.3.4. Zero Flow Rate (ZFR) for estimation of in vivo recovery...........................47

2.3.5. Increased ECF penetration of erlotinib and OSI-420 in $\mathrm{Abcg} 2^{-/ /}$and Mdrla $/ b^{-/} \mathrm{Abcg} 2^{-/-}$mice.....................................................................4

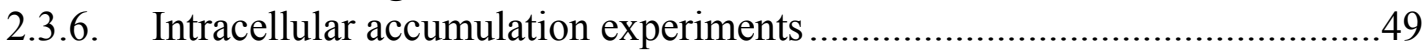

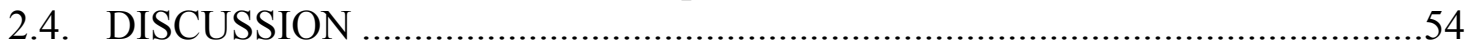

\section{CHAPTER 3. MAGNETIC RESONANCE IMAGING (MRI)-GUIDED MICRODIALYSIS CANNULA IMPLANTATION IN A SPONTANEOUS HIGH-GRADE GLIOMA MURINE MODEL* ............................................................60}

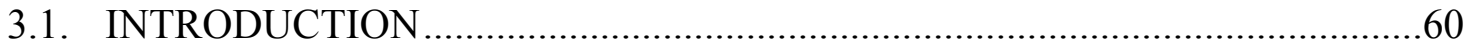

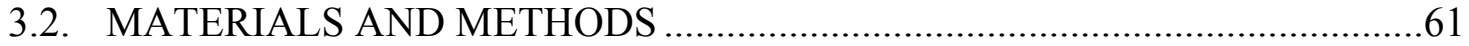

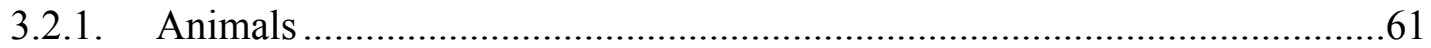

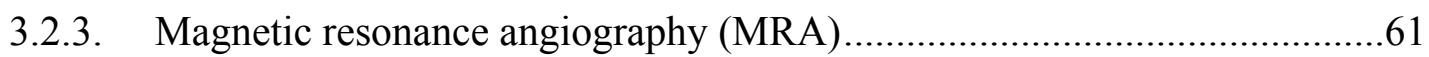

3.2.2. Magnetic resonance imaging (MRI) …………..........................................62

3.2.4. Stereotactic surgery for microdialysis cannula insertion ..............................62

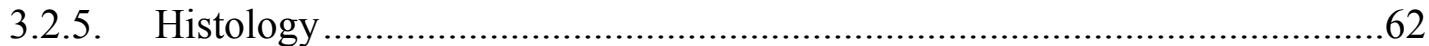

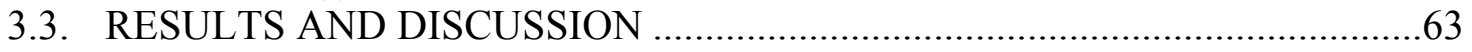

3.3.1. Identification of the rostral rhinal vein as a correlative reference point ......63

3.3.2. Determination of tumor coordinates and confirmation of probe placement

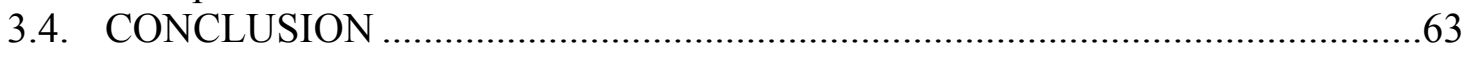

\section{CHAPTER 4. CEREBRAL MICRODIALYSIS FOR SIMULTANEOUS SAMPLING OF TWO TYROSINE KINASE INHIBITORS IN AN ORTHOTOPIC MOUSE MODEL OF GLIOMA............................................................68}

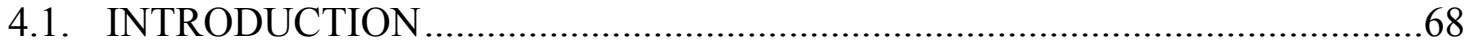

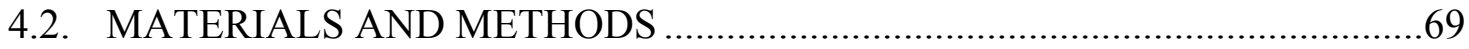


4.2.1. Chemicals and reagents

4.2.2. Liquid chromatography/tandem mass spectrometry (LC MS/MS) method for crenolanib determination in mouse plasma and CSF samples ..69

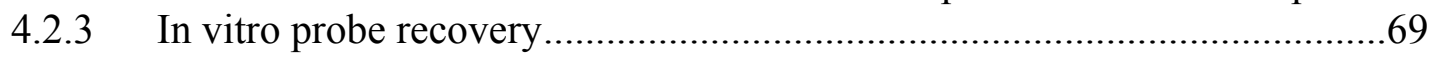

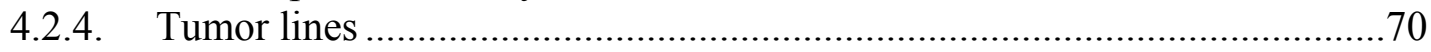

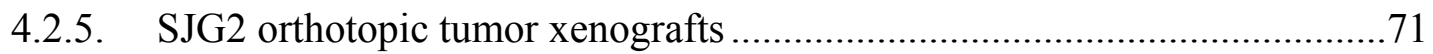

4.2.6. Magnetic Resonance Imaging (MRI) .......................................................

4.2.7. Determination of crenolanib protein binding in mouse plasma....................71

4.2.8. Crenolanib and erlotinib steady state plasma pharmacokinetics ..................71

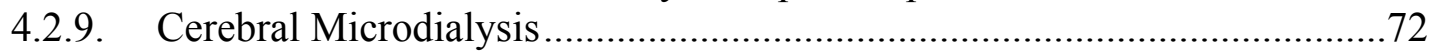

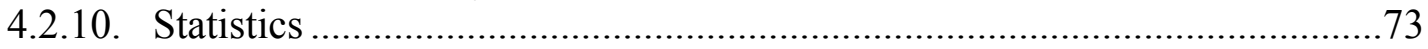

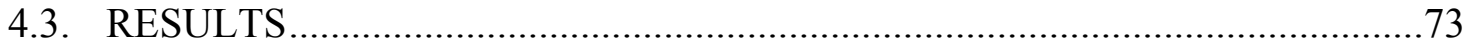

4.3.1. HPBCD improves crenolanib recovery in vitro ...........................................73

4.3.2. Tumor growth kinetics and morphology .....................................................77

4.3.3. Crenolanib and erlotinib steady state plasma pharmacokinetics ..................77

4.3.4. Crenolanib protein binding in mouse plasma …………..............................77

4.3.5. Crenolanib penetration in SJG2 xenografts ...............................................77

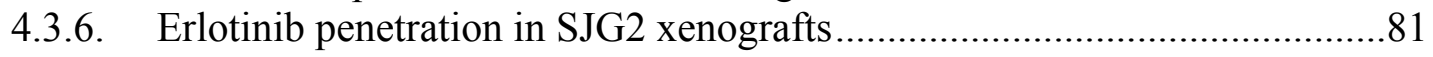

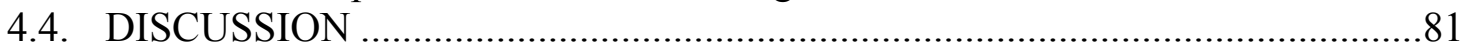

CHAPTER 5. SUMMARY, DISCUSSION AND FUTURE DIRECTIONS...............85

LIST OF REFERENCES ..................................................................................................92

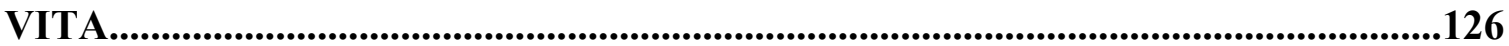




\section{LIST OF TABLES}

Table 1-1. Summary for important genetic alteration in adult and pediatric malignant glioma

Table 2-1. Unbound fractions ( $f u)$ in plasma of different mouse strains

Table 2-2. Pharmacokinetic parameters for different mice strains

Table 2-3. In vitro recovery values (\%) obtained while changing perfusion flow rates in a similar fashion to in vivo experiment to recoveries obtained with stable flow rate. .56

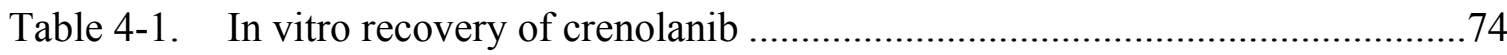

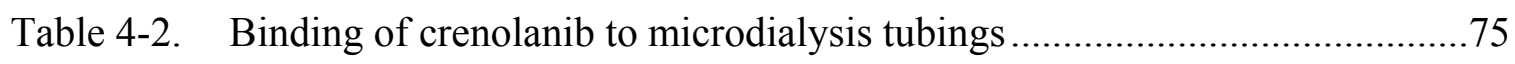




\section{LIST OF FIGURES}

Figure 1-1. Schematic presentation of signaling pathways targeted by molecular therapies in malignant glioma....

Figure 1-2. Schematic representation of the capillary endothelial cells that build up the BBB

Figure 1-3. Schematic representation of the BBB .......................................................

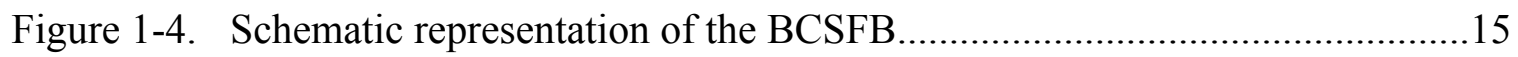

Figure 1-5. Diagrammatic illustration of the retrodialysis method ..................................32

Figure 2-1. Compartmental pharmacokinetic model for erlotinib and OSI-420 …..........42

Figure 2-2. Plasma concentration-time plots of erlotinib and OSI-420 after oral administration of a single dose of $50 \mathrm{mg} / \mathrm{kg}$ erlotinib.

Figure 2-3. Plasma concentration-time plots on linear scale of erlotinib and OSI-420 after oral administration of a single dose of $50 \mathrm{mg} / \mathrm{kg}$ erlotinib

Figure 2-4. In vitro microdialysis studies .48

Figure 2-5. Brain penetration of erlotinib and OSI-420 after a single oral dose of erlotinib

Figure 2-6. H\&E staining showing the microdialysis cannula and probe track in brain ECF with no gross pathological effect of 10\% HPBCD on brain tissue.

Figure 2-7. Erlotinib and OSI-420 intracellular accumulation in vitro in cell lines expressing efflux transporters.

Figure 2-8. Intracellular accumulation data from $15 \mathrm{~min}$ and $30 \mathrm{~min}$ time-points combined.

Figure 2-9. Transport of erlotinib, A and OSI-420, B by human organic ion transporters

Figure 2-10. Proposed model for the role of efflux and uptake transporters in erlotinib CNS penetration

Figure 3-1. Presence of the rostral rhinal vein on the surface of a mouse brain

Figure 3-2. Derivation of MRI-guided stereotactic coordinates for cannula implantation in spontaneously arising brain tumors 
Figure 4-1. Validation of the ZFR method ......................................................... 76

Figure 4-2. Growth characteristics of SJG2 tumors .............................................. 78

Figure 4-3. Concentration-time profile for crenolanib, erlotinib, and OSI-420 after implantation of mini-osmotic pumps in CD1 nude mice ..........................79

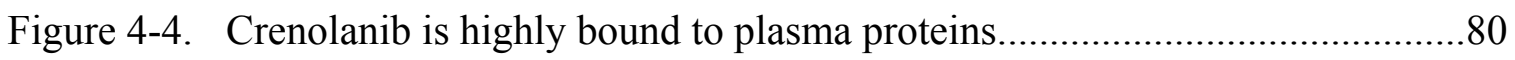

Figure 4-5. Crenolanib and erlotinib penetration in SJG2 xenografts..........................82

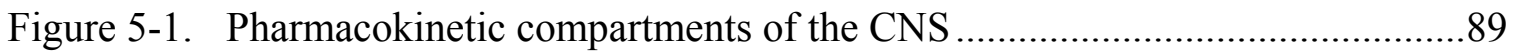




\section{LIST OF ABBREVIATIONS}

\begin{tabular}{ll} 
AA & Anaplastic astrocytoma \\
ABC & ATP-binding cassette \\
aCSF & Artificial cerebrospinal fluid \\
AIC & Akaike's information criterion \\
ATP & Adenosine triphosphate \\
AUC & Area under the curve \\
BBB & Blood-brain barrier \\
BCRP & Breast cancer resistance protein \\
BCSFB & Blood cerebrospinal fluid barrier \\
bFGF & Basic fibroblast growth factor \\
BSA & Bovine serum albumin \\
CBTRUS & Central brain tumor registry of the United States \\
CL & Clearance \\
CMV & Cytomegalo virus \\
CNS & Central nervous system \\
CSF & Cerebrospinal fluid \\
CT & Computed tomography \\
DMEM & Dulbecco's modified eagle's medium \\
DMSO & Dimethyl sulfoxide \\
ECF & Extracellular fluid \\
EGF & Epidermal growth factor \\
EGFR & Epidermal growth factor receptor \\
FGF & Fibroblast growth factor \\
Fu & Unbound fraction \\
GBM & Glioblastoma multiforme \\
GEMM & Genetically engineered mouse model \\
GFAP & Glial fibrillary acidic protein \\
HPBCD & 2-Hydroxypropyl- $\beta$-cyclodextrin \\
IIV & Inter-individual variability \\
LC MS/MS & Liquid chromatography/tandem mass spectrometry \\
LLOQ & Lower limit of quantification \\
MAPK & Mitogen-activated protein kinase \\
MDCK & Madin-Darby canine kidney epithelial \\
MDR & Multiple drug resistance \\
MRI & Magnetic resonance imaging \\
mRNA & Messenger RNA \\
MRP1 & Multidrug resistance protein 1 \\
NSCLC & Non-small cell lung cancer \\
OAT & Organic anion transporter \\
OATP & Organic anion-transporting polypeptide \\
OCT & Organic cation transporter \\
OFV & Objective function value \\
PDGF & Platelet-derived growth factor \\
& \\
\hline
\end{tabular}


PDGFR

PDGFR $\alpha$

PDGFRA

P-gp

PI3K

PTEN

RTK

SLC

TCGA

vCSF

VEGF

VEGFR

WHO

ZFR
Platelet-derived growth factor receptor

Platelet-derived growth factor receptor alpha protein

Platelet-derived growth factor receptor alpha gene

P-glycoprotein

Phosphatidyl inositol 3-kinase

Phosphate and tensin homolog

Receptor tyrosine kinase

Solute carrier

The cancer genome atlas project

Ventricular cerebrospinal fluid

Vascular endothelial growth factor

Vascular endothelial growth factor receptor

World Health Organization

Zero flow rate 


\section{CHAPTER 1. INTRODUCTION}

\subsection{INTRODUCTION OF MALIGNANT GLIOMA}

\subsubsection{Incidence of malignant glioma in adult and pediatric populations}

Malignant gliomas are a heterogeneous group of tumors that represents about $70 \%$ of adult central nervous system (CNS) tumors (Porter et al., 2010). Approximately $75 \%$ of adult gliomas are malignant and can be categorized in two histological subclasses: anaplastic astrocytoma (AA; World Health Organization WHO grade III) and glioblastoma multiforme (GBM; WHO grade IV). AA and GBM are collectively referred to as high-grade gliomas (Fuller, 2008). According to the central brain tumor registry of the United States (CBTRUS), more than 15,000 adult patients are diagnosed with malignant gliomas each year (CBTRUS, 2010). Conversely, malignant childhood gliomas are less common than their adult counterparts, comprising only $15-20 \%$ of all childhood CNS tumors (Broniscer and Gajjar, 2004). The reported incidence of malignant glioma in both adults and pediatric populations has increased over the past 30 years, partially due to improved diagnosis with novel imaging modalities such as computed tomography (CT) and magnetic resonance imaging (MRI) (Legler et al., 1999; Smith et al., 1998).

The anatomical location of these tumors differs in adults and children. In adults, the majority of malignant gliomas develop in the subcortical white matter of the cerebral lobes $(86 \%)$ while the rest develops either in the deep structures of the cerebrum, the ventricles, or in the cerebellum (Larjavaara et al., 2007). On the other hand, pediatric gliomas arise from the supratentorial hemispheres or from the pons with the later known as diffuse brainstem glioma. Supratentorial tumors represent $6-12 \%$ of all pediatric primary CNS tumors while diffuse brainstem glioma accounts for 3-9\% (Broniscer and Gajjar, 2004).

\subsubsection{Pathological features of malignant glioma}

Depending on the course of the disease, malignant gliomas can be categorized into primary tumors that develop de novo in the brain with no prior tumor lesion, or secondary tumors that progress from a low grade to a higher grade astrocytoma (Furnari et al., 2007). Both adult and pediatric glioma arise predominantly de novo while secondary tumors are rare (Broniscer et al., 2007a; Furnari et al., 2007; Phuphanich et al., 1984; Wen and Kesari, 2008).

Malignant gliomas are characterized by their histological heterogeneity. AA presents clinically with abnormally high mitotic activity and increased cellularity. GBM also displays similar, or higher, hypercellularity in addition to the highly hypoxic nature of these tumors (Fuller, 2008). GBM is distinguished from AA via two characteristic features. First, GBM tumors exhibit micronecrotic foci surrounded with pseudopalisading 
cells (Rong et al., 2006). The second characteristic feature of GBM is its high angiogenic propensity and intense microvascular hyperplasia (Fuller, 2008; Miyagami and Katayama, 2005). This hyperplastic vasculature develops in response to pro-angiogenic growth factors, such as vascular endothelial growth factor (VEGF), that induce endothelial cell survival, proliferation, and migration (Plate et al., 1994). These microvessels are structurally and functionally immature, which may contribute to poor tumor oxygenation and hypoxic microenvironment (Miyagami and Katayama, 2005). The formation of new blood vessels is crucial for glioma development and progression. This was supported by reports showing that high degree of microvascular proliferation correlates with low overall survival in adult glioma patients (Bello et al., 2004; Cheng et al., 1996; Leon et al., 1996).

An important histological feature of high-grade gliomas is the highly invasive propensity of glioma cells as they metastasize and invade either along endothelial cells or more frequently through the brain parenchyma. This diffuse infiltrative phenotype is referred to as gliomatosis cerebri (Bendszus et al., 2000). Because of this infiltrative growth pattern, complete surgical resection of malignant glioma is not possible, and local tumor recurrence is inevitable (Giese et al., 2003).

\subsubsection{Genetic and molecular alterations characteristic of malignant glioma}

In the past five years, studies conducting comprehensive genomic and protein expression profiling of adult and pediatric malignant glioma revealed differences in gene copy number alterations and transcriptional profiles (Parsons et al., 2008; Paugh et al., 2010a; TCGA, 2008) (Table 1-1). These studies revealed that the function of several tumor suppressor pathways such as P53 and retinoblastoma $(\mathrm{Rb})$ is commonly mutated or deleted in both adult and pediatric gliomas (Newcomb et al., 2000; Pollack et al., 2001; Suri et al., 2009; TCGA, 2008). Alteration of multiple receptor tyrosine kinases (RTKs) signaling pathways is another frequent genetic finding in malignant gliomas (Parsons et al., 2008; Paugh et al., 2010a; Stommel et al., 2007; TCGA, 2008). RTKs are a family of cell surface receptors that can be activated by extracellular ligands. Upon activation, RTKs phosphorylate tyrosine residues on several target proteins resulting in their activation (Fan and Weiss, 2010; Robinson et al., 2000). The signal initiated by RTKs is then transduced and amplified through intracellular downstream pro-survival signaling cascades, such as the phosphatidyl inositol 3-kinase/Akt (PI3K/Akt) pathway or the mitogen-activated protein kinase (MAPK) pathway, leading to increased tumor cell survival, migration, and invasiveness (Fan and Weiss, 2010; Ren et al., 2007) (Figure 1-1). RTKs shown to play a role in the development, progression, or therapeutic resistance of malignant glioma include the epidermal growth factor receptor (EGFR), platelet-derived growth factor receptor (PDGFR) and vascular endothelial growth factor receptor (VEGFR) (Ayuso-Sacido et al., 2010; Paugh et al., 2010a; Raymond et al., 2000; Squatrito and Holland, 2011).

About half of adult glioma cases exhibit mutation or amplification in EGFR (TCGA, 2008). Deletion or mutation of the phosphate and tensin homolog tumor 
Table 1-1. Summary for important genetic alteration in adult and pediatric malignant glioma

\begin{tabular}{|c|c|c|}
\hline Gene & $\begin{array}{l}\text { Alteration in adult glioma } \\
\text { patients }\end{array}$ & $\begin{array}{l}\text { Alteration in pediatric glioma } \\
\text { patients }\end{array}$ \\
\hline$E G F R$ & $\begin{array}{l}\text { Amplification (25\%) (Parsons et } \\
\text { al., 2008; TCGA, 2008) } \\
\text { Mutation (3.3\%) (TCGA, 2008) } \\
\text { Mutation and amplification } \\
(17.5 \%)(\text { TCGA, 2008) }\end{array}$ & $\begin{array}{l}\text { Amplification (rare) (0-7\%) } \\
\text { (Bredel et al., 1999; Paugh et al., } \\
\text { 2010a; Pollack et al., 2006; Sung } \\
\text { et al., 2000; Suri et al., 2009) }\end{array}$ \\
\hline PDGFRA & $\begin{array}{l}\text { Amplification }(13 \%)(T C G A \\
2008)\end{array}$ & $\begin{array}{l}\text { Amplification (14\%) (Paugh et } \\
\text { al., 2010a) }\end{array}$ \\
\hline$c-M E T$ & $\begin{array}{l}\text { Amplification (4\%)(TCGA, } \\
\text { 2008) }\end{array}$ & $\begin{array}{l}\text { Amplification (rare in GBM 3\% } \\
\text { but more common in diffuse } \\
\text { intrinsic pontine glioma 26\%) } \\
\text { (Paugh et al., 2010a) }\end{array}$ \\
\hline PTEN & $\begin{array}{l}\text { Mutation, homozygous deletion } \\
(36 \%) \text { (Parsons et al., 2008; } \\
\text { TCGA, 2008) }\end{array}$ & $\begin{array}{l}\text { Deletion (rare) }(0-5 \%) \text { (Pollack et } \\
\text { al., 2006; Suri et al., 2009) }\end{array}$ \\
\hline P53 Pathway & $\begin{array}{l}\text { TP53: Mutation, homozygous } \\
\text { deletion (35\%) (TCGA, 2008) } \\
\text { Genes that regulate p53 stability: } \\
M D M 2 \text {, amplified in } 14 \% \text {, } \\
M D M 4 \text {, amplified in } 7 \% \\
C D K N 2 A \text {, homozygous deletion } \\
\text { or mutation in } 52 \% \\
\text { Collectively, this pathway is } \\
\text { targeted in } 87 \% \text { of cases (TCGA, } \\
2008 \text { ) }\end{array}$ & $\begin{array}{l}\text { Mutation (34\%)(Pollack et al., } \\
\text { 2001) }\end{array}$ \\
\hline RB Pathway & $\begin{array}{l}R B 1 \text { : Mutation, homozygous } \\
\text { deletion }(11 \%) \text { (TCGA, 2008) } \\
\text { Genes that regulate Rb activity: } \\
C D K N 2 A \text {, deletion or mutation } \\
\text { in } 52 \% \\
C D K N 2 B \text {, deletion in } 47 \% \\
\text { Collectively, this pathway is } \\
\text { altered in } 78 \% \text { of cases }\end{array}$ & $\begin{array}{l}C D K N 2 A, \text { homozygous deletion } \\
(10 \%)(\text { Newcomb et al., } 2000)\end{array}$ \\
\hline
\end{tabular}




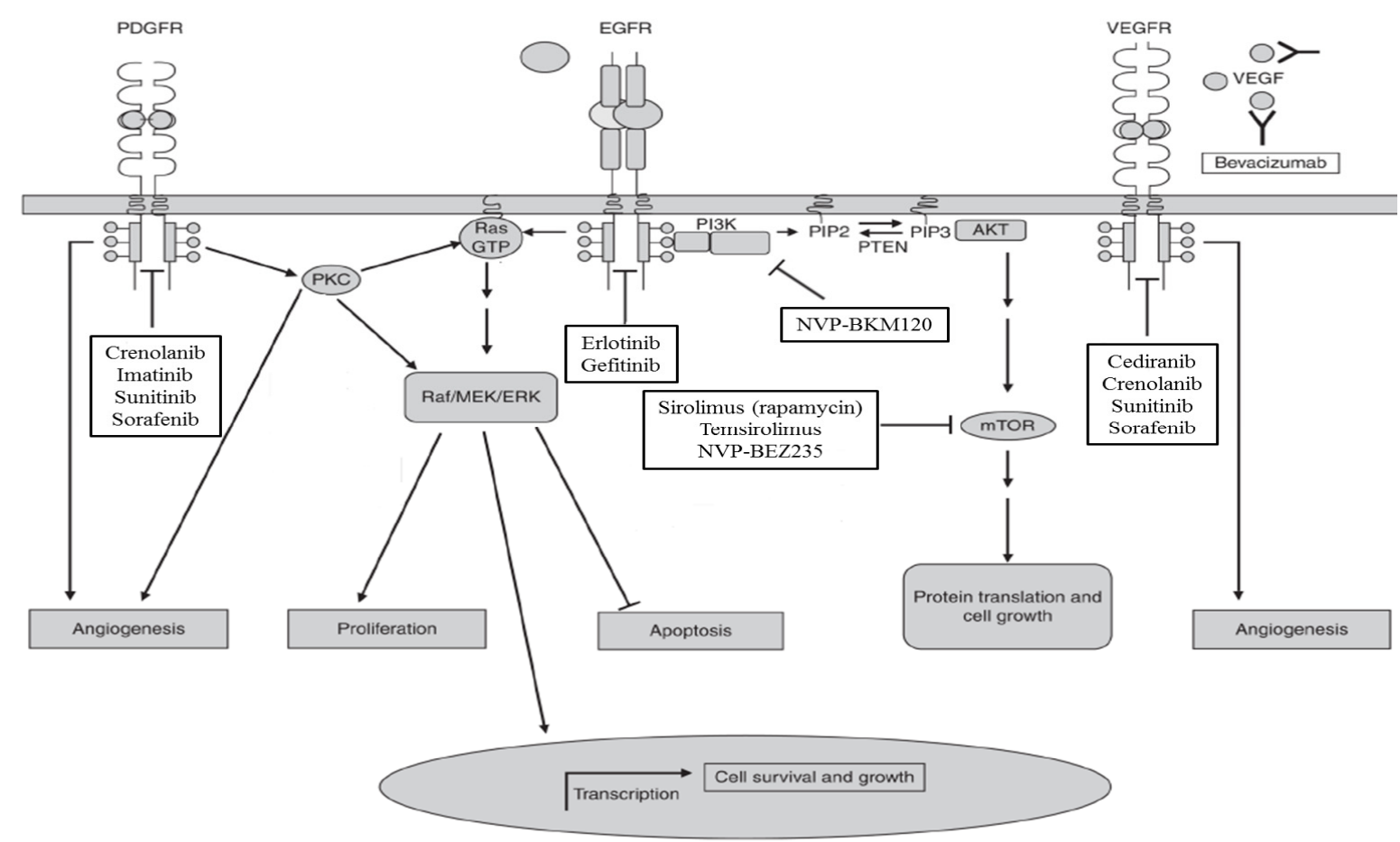

Figure 1-1. Schematic presentation of signaling pathways targeted by molecular therapies in malignant glioma

The diagram illustrates pathways that are activated upon RTK activation. PDGFR, EGFR, and VEGFR activation share common intracellular effectors. Arrowheads indicate signal transduction while a blunted end indicates inhibition of a target.

Source: Adapted by permission from Springer. Mercer RW et al. (2009) Targeted Therapies for Malignant Glioma Progress and Potential. BioDrugs 23:25-35. 
suppressor gene (PTEN), the major inhibitor of PI3K/Akt signaling pathway is found in $36 \%$ of these tumors (TCGA, 2008). In contrast, pediatric glioma cases rarely exhibit EGFR gene amplification or PTEN gene deletion or mutation (0-5\%) (Paugh et al., 2010a; Pollack et al., 2006; Sung et al., 2000; Suri et al., 2009). Despite the low frequency of EGFR amplifications in childhood glioma, EGFR protein overexpression is observed in up to $85 \%$ of the cases (Bredel et al., 1999; Gilbertson et al., 2003; Pollack et al., 2006; Thorarinsdottir et al., 2008).

In pediatric gliomas, platelet-derived growth factor alpha (PDGFRA) is the predominant target of focal amplification (Paugh et al., 2010a). The extent of PDGFR $\alpha$ activation, measured by the receptor phosphorylation of several tyrosine residues, is significantly associated with the malignant histology of childhood gliomas (Thorarinsdottir et al., 2008). Other receptor tyrosine kinases, such as c-MET, may be altered in adult and pediatric gliomas (Paugh et al., 2010a; TCGA, 2008). The different frequencies of genetic alterations between adult and pediatric gliomas reflects the complexity and heterogeneity of these tumors and indicates the need for molecularly targeted therapies directed towards the aberrant signaling pathways in individual tumors.

The activation status of critical glioma survival pathways, such as PI3K/Akt, is determined by redundant inputs from multiple receptor tyrosine kinases (RTKs) such as EGFR, PDGFR, and c-MET. The signals provided by these RTKs can be either used simultaneously or sequentially by glioma cells to maintain an active downstream $\mathrm{PI} 3 \mathrm{~K} / \mathrm{Akt}$ signaling and thus inhibiting PI3K/Akt signaling may require targeting multiple RTKs (Stommel et al., 2007).

\subsubsection{Current approaches for treatment of malignant glioma}

The prognosis and clinical outcome for AA patients is better than those diagnosed with GBM (Broniscer et al., 2005; Finlay et al., 1995; Rutten et al., 1981). Despite aggressive treatment modalities, including surgical resection, radiation, and chemotherapy, the median overall survival of patients with newly diagnosed GBM is less than 4 years for children and 1.5 years for adults (Minniti et al., 2008; Song et al., 2010; Stupp et al., 2005). The infiltrative nature of malignant glioma allows these tumors to invade and metastasize through the brain parenchyma. Ultimately, this invasive propensity leads to failure of surgical resection to achieve complete tumor removal (Amberger-Murphy, 2003; Giese et al., 2003).

For adult patients newly diagnosed with malignant glioma, the standard treatment regimen for adults is radiotherapy ( 2 Gray per day 5 days per week for 6 weeks, for a total of 60 Gray), plus continuous daily administration of temozolomide, a DNA alkylating agent. This is followed by six cycles of temozolomide (150 to $200 \mathrm{mg}$ per square meter for 5 days during each 28 -day cycle). This regimen modestly improved the median survival of GBM patients to 14.6 months as compared to 12.1 months with radiotherapy alone (Stupp et al., 2007; Stupp et al., 2005). 
Given the highly vascular nature of malignant glioma, angiogenesis inhibitors, especially those targeting VEGF signaling, have been used alone or in combination with chemotherapeutic agents for the treatment of adult recurrent glioblastoma. A multi-center phase II study reported a notable improvement of the 6-month progression-free survival in patients with recurrent GBM treated with bevacizumab, a VEGF monoclonal antibody, when compared to historical controls treated with irinotecan alone (Friedman et al., 2009). The impressive results reported in this study led to the accelerated US Food and Drug Administration approval of bevacizumab for treatment of adult patients with recurrent glioblastoma.

In contrast to their adult counterparts, pediatric glioma patients showed poor response to the standard of care of the adult population. The administration of temozolomide after radiation therapy failed to improve the poor prognosis of children with malignant glioma (Broniscer and Gajjar, 2004; Broniscer et al., 2005). Further, disappointing results were reported in trials using bevacizumab and irinotecan in treatment of children with recurrent malignant glioma (Gururangan et al., 2010). For pediatric glioma patients, maximum safe surgical resection and radiation therapy is the mainstay of treatment. The striking differences between the pediatric and adult populations in their response to several therapeutic regimens, despite their histological similarities, support the use of regimens that target specific pathways altered in each tumor (Broniscer and Gajjar, 2004).

\subsubsection{Emerging treatments for malignant glioma}

The advances in our understanding of the genetic alterations of these tumors allow for the identification of molecular targets that are important for tumor survival and progression. Aberrant signaling through RTKs such as EGFR, PDGFR $\alpha$, and VEGFR results in activation of downstream effectors that promote cellular proliferation, survival, invasiveness, and inhibits apoptosis of glioma cells (Ayuso-Sacido et al., 2010; Hatanpaa et al., 2010; Sharma et al., 2011). Thus, inhibiting these pathways represent a reasonable therapeutic strategy in attempts to inhibit tumor growth.

\subsubsection{Inhibitors of EGFR pathway}

Alteration of the EGFR signaling pathway is a common feature in adult glioma in the form of gene amplification or mutation (TCGA, 2008), and in pediatric glioma in the form of protein overexpression (Bredel et al., 1999; Gilbertson et al., 2003; Pollack et al., 2006; Thorarinsdottir et al., 2008). These findings make EGFR an attractive target in the management of malignant glioma.

Gefitinib (Iressa, Astra Zeneca) and erlotinib (Tarceva, OSI Pharmaceuticals) are both quinazolin-4-amine derivatives with specific and potent inhibitory activity against EGFR. Both gefitinib and erlotinib competitively bind to the cytoplasmic ATP-binding domain of EGFR and inhibits its tyrosine kinase activity. This prevents EGFR 
phosphorylation and interrupts its downstream signaling cascade (Raymond et al., 2000). In vitro, erlotinib was shown to suppress anchorage-independent growth and induce apoptosis in glioma cell lines (Halatsch et al., 2004).Only erlotinib, not gefitinib, can inhibit the most common type of EGFR mutation, EGFRvIII, which lacks a portion of the extracellular ligand binding domain rendering EGFR constitutively active (Heimberger et al., 2002; Peereboom et al., 2010).

EGFR inhibitors were shown to reduce tumor growth and prolong survival in subcutaneous xenograft models of high-grade glioma (Ciardiello et al., 2001; Heimberger et al., 2002). However, neither gefitinib nor erlotinib showed clinical benefit in improving the overall survival in adult or pediatric patients with high-grade glioma (Geoerger et al., 2011; Peereboom et al., 2010; Prados et al., 2009; Raizer et al., 2010b; Reardon et al., 2006; Rich et al., 2004; van den Bent et al., 2009a).This might be explained by previous reports indicating that targeting EGFR only is insufficient for inhibiting downstream PI3K/Akt signaling and that more effective therapy may require targeting of multiple RTKs (Stommel et al., 2007).

\subsubsection{Inhibitors of PDGFR pathway}

The two isoforms PDGF-A and B homo or hetero-dimerize, bind to, and induce the activation of PDGFR $\alpha$ or $\beta$. Activated PDGFR $\alpha$ or PDGFR $\beta$ then activate PI3K/Akt and MAPK pathways (Liu et al., 2011). PDGFR $\alpha$ or $\beta$ are commonly overexpressed in glioma (Guha et al., 1995; Liang et al., 2008; Mauro et al., 1991; Nister et al., 1991; Thorarinsdottir et al., 2008) and are thought to play an important role in tumor invasiveness and angiogenesis (Pietras et al., 2003; Thorarinsdottir et al., 2008).

Imatinib (Gleevec, Novartis) is an RTK inhibitor that targets Bcr-Abl and c-kit. Imatinib also inhibits PDGFR activation though at concentrations higher than that required for Bcr-Abl and c-kit inhibition (Mahboobi et al., 2008). Imatinib showed promising results in xenografts derived from the human glioma cell lines U343 and U87-MG. In these studies, oral administration of imatinib prolonged the survival of subcutaneous xenograft mouse models with PDGFR-expressing tumors (Kilic et al., 2000). The excitement resulting from these preclinical data faded when disappointing results were reported in phase II studies in recurrent glioma patients. The reports showed that imatinib failed to prolong the 6 months progression free survival of recurrent glioma patients (Raymond et al., 2008; Wen et al., 2006). Furthermore, a Phase III study comparing the efficacy of the combination of imatinib and adjuvant chemotherapy versus chemotherapy alone in heavily pretreated malignant glioma patients showed similar median progression free survival in both treatment arms which indicates limited clinical benefit of the combination (Dresemann et al., 2010).

Reports showing limited CNS penetration of imatinib, together with its low potency and lack of specificity for PDGFR inhibition, raised some questions on whether imatinib is a suitable drug for treatment of malignant glioma and suggested the need for more specific and potent PDGFR inhibitors with better CNS penetration profiles (Mahboobi et 
al., 2008; Neville et al., 2004). Fortunately, specific inhibitors of the PDGFR pathway, such as crenolanib, are available (AROG Pharmaceuticals, LLC). Crenolanib is an orally bioavailable, highly specific, and potent PDGFR inhibitor that targets both $\alpha$ and $\beta$ receptor types (Lewis et al., 2009). Crenolanib is more than 100-fold more selective towards PDGFR than other kinases, such as c-KIT, VEGFR-2, and EGFR (Lewis et al., 2009). Crenolanib (50 mg/kg twice daily) resulted in $47 \%$ inhibition of tumor growth in U87-MG human glioma subcutaneous xenografts (AROG Pharmaceuticals, LLC, Investigator's Brochure Crenolanib Besylate March 2011). To our knowledge, crenolanib CNS penetration has not been reported to date. Currently, crenolanib is undergoing phase I trials in children and phase II trials in adult patients with malignant glioma.

\subsubsection{Inhibitors of VEGFR pathway}

The development of neovasculature, angiogenesis, is an important process for the growth and invasiveness of malignant glioma. The high angiogenic capacity of glioma is primarily mediated by VEGF although other proangiogenic factors, such as PDGF and fibroblast growth factor (FGF), can also contribute (Guo et al., 2003; Plate et al., 1992; Schmidt et al., 1999). VEGF binds to its receptors, mainly VEGFR-2, on tumor vessels resulting in increased vascular permeability and enhanced endothelial cell proliferation, survival, and migration. Distinct structural and functional abnormalities differentiate the newly formed tumor vessels from normal vessels (Plate et al., 1992). Tumor endothelial cells are tortuous with large fenestrations, abnormal basement membrane, and reduced pericyte coverage (Jain, 2005; Morikawa et al., 2002). Thus, despite the increased microvessel density in glioma, the blood flow in these vessels is heterogeneous and sluggish which leads to hypoxia, acidosis, necrosis, and elevated tumor interstitial pressure (Fukumura and Jain, 2007; Hanahan and Folkman, 1996; Jain, 2001; 2005).

Given the highly angiogenic nature of malignant glioma, targeting angiogenic signaling pathways was shown to be a reasonable therapeutic strategy for these tumors (Batchelor et al., 2007; Brastianos and Batchelor, 2010; Kamoun et al., 2009). Inhibitors of the VEGF signaling pathway are the most clinically developed of the anti-angiogenic therapies. Two strategies to target VEGF signaling pathway are currently being tested; the first is targeting the ligand, VEGF, using VEGF monoclonal antibodies such as bevacizumab (Avastin, Genentech-Roche), and the second is targeting the VEGF receptor expressed on capillary endothelial cells, VEGFR2 (McMahon, 2000; Reardon et al., 2011).

Recent findings showed that bevacizumab in combination with irinotecan improved the 6 months progression free survival of patients with recurrent GBM to $46 \%$ compared to $21 \%$ with historical controls, indicating potential benefit of anti-angiogenic agents (Vredenburgh et al., 2007). More than half the patients on this trial had at least a partial radiological response measured by the extent of tumor enhancement on T1-weighted MRI scans (Vredenburgh et al., 2007). 
Several small molecules targeting VEGFR have been developed such as cediranib (Recentin, AstraZeneca). Cediranib, a pan inhibitor of VEGFR, successfully increased survival and led to vascular normalization and edema alleviation in orthotopic glioma mouse models despite continuous tumor enlargement in the cediranib-treated group (Kamoun et al., 2009). A similar, though less pronounced effect, was observed upon treating these mice with dexamethasone. These findings suggest that the effect observed with cediranib treatment is more likely to be associated with edema alleviation than with direct anti-tumor effect (Kamoun et al., 2009). In phase II trials for adult patients with recurrent glioblastoma, cediranib showed improved 6 months progression free survival (26\%), though overall survival was similar to historical controls. Tumor vascular normalization was rapidly reversed upon interruption of the cediranib regimen (Batchelor et al., 2007).

Ultimately, VEGFR pathway inhibitors produced only modest improvement in the overall survival in patients with malignant glioma. This raised the question of whether the observed improvement in radiological response and progression free survival is due to a genuine anti-tumor effect or merely a result of reduced vascular permeability that limits tumor enhancement on T1-weighted MRI. Thus, some oncologists advocate reconsidering the anti-edematous effect of bevacizumab as an expensive steroid-like effect (van den Bent et al., 2009b; Wick et al., 2010).

\subsubsection{Challenges facing molecularly targeted therapies in malignant glioma}

In general, results from molecularly targeted agents in treatment of malignant glioma patients have been disappointing despite some encouraging in vitro and preclinical data. No major survival advantage has been observed with any of these agents in phase II or III trials (Batchelor et al., 2007; Dresemann et al., 2010; Peereboom et al., 2010; Raizer et al., 2010b; Rich et al., 2004). The failure of these agents may be explained by several resistance mechanisms. These include coactivation of multiple receptor tyrosine kinases that provide redundant signals to maintain active downstream signaling. Coactivation of three or more RTKs that include EGFR, PDGFR $\alpha$, and c-MET was demonstrated in 19/20 glioma cell lines (Stommel et al., 2007). Inhibition of downstream PI3K signaling could only be accomplished when all activated receptors were targeted while inhibiting single RTK did not affect downstream signaling (Stommel et al., 2007; Thaker and Pollack, 2009). Thus, understanding the pathways activated in individual patients and simultaneously targeting multiple components of the PI3K pathway using combination regimens may provide clinical benefit for patients with malignant glioma (Mercer et al., 2009; Stommel et al., 2007; Thaker and Pollack, 2009).

Further, resistance of malignant glioma to some tyrosine kinase inhibitors can be explained by the deletion of key tumor suppressor proteins such as PTEN, a genetic alteration almost exclusive to adult glioma patients, leading to constitutive receptorindependent activation of the PI3K/Akt pathway (Mellinghoff et al., 2005). Recently, the genetic complexity of these tumors was recently highlighted in microarray studies that identified several molecular subclasses of GBM where each of these subclasses responds 
differently to treatment indicating that malignant gliomas are more complex than being just a single disease type (Phillips et al., 2006; Verhaak et al., 2010).

The effectiveness of molecularly targeted therapies depends upon achieving sufficient concentrations of these agents in the tumor for adequate duration. To induce a therapeutic effect, the plasma protein-unbound portion of these agents should access the relevant target site within the tumor. The concentration of unbound drug achieved within the tumor depends on several factors, which include absorption, distribution, metabolism, and elimination (i.e., pharmacokinetics) of the drug, the toxicity associated with its administration, the extent of plasma protein binding, and the ability of the drug to cross the blood-brain barrier (BBB). The BBB limits tumor penetration of these agents and thus may provide plausible explanation for the lack of efficacy of drugs with limited CNS and tumor penetration (Tredan et al., 2007; Westerhout et al., 2011). Moreover, the distribution of anti-cancer drug throughout the tumor is non-uniform (Jain, 2005). Uniform distribution of effective concentration of the anti-cancer drug throughout the tumor may allow the anti-cancer agent to encounter more tumor cells and thus improve its anti-tumor activity.

\subsection{BARRIERS AGAINST ANTI-CANCER DRUG CNS PENETRATION}

The brain is protected by a dynamic barrier that maintains the stability of the brain function by separating the brain from the systemic circulation and hence the name bloodbrain barrier (BBB) (Figure 1-2) (Rubin and Staddon, 1999). The BBB plays an important role in protecting the sanctuary of the brain and its normal development from circulating toxins and harmful chemicals. As a result, the brain will have limited exposure to drugs intended to treat neurological diseases and brain tumors (McCarty, 2005; Rubin and Staddon, 1999).

The BBB is composed of capillary endothelial cells which are supported by pericytes, astrocytes, and microglial cells (McCarty, 2005). These endothelial cells are connected to each other by a number of integral membrane proteins such as occludins, caludins, and ZO 1-3, forming tight junctions that block the paracellular (between cell) transport of xenobiotics including anti-cancer drugs (Figure 1-2) (Kniesel and Wolburg, 2000; Wolburg and Lippoldt, 2002). To overcome this paracellular impermeability, drugs have to pass through the endothelial cells (i.e., trans-cellular route) to reach the brain (Pardridge, 1999; Terasaki and Ohtsuki, 2005).

Some drugs with high lipophilicity, low polar surface area, and low molecular weight can pass through the transcellular route via passive diffusion. However, several transport proteins on the endothelial cells can limit the brain penetration of these molecules despite their favorable physicochemical properties (Eros et al., 2002; Mahar Doan et al., 2002; McCarty, 2005; Pardridge, 1998; Rubin and Staddon, 1999). Members of the ATP-binding cassette (ABC) transporter family are efflux pumps expressed on the endothelial cells. These pumps transport molecules across the BBB into the blood against their concentration gradient by utilizing energy from ATP hydrolysis (PL and GM, 2003; 


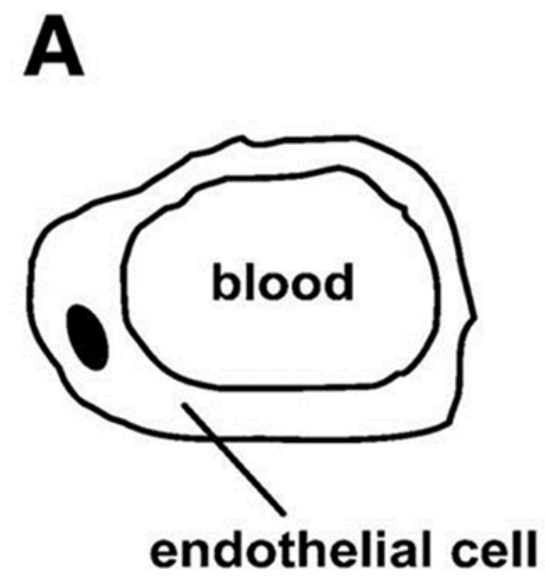

B

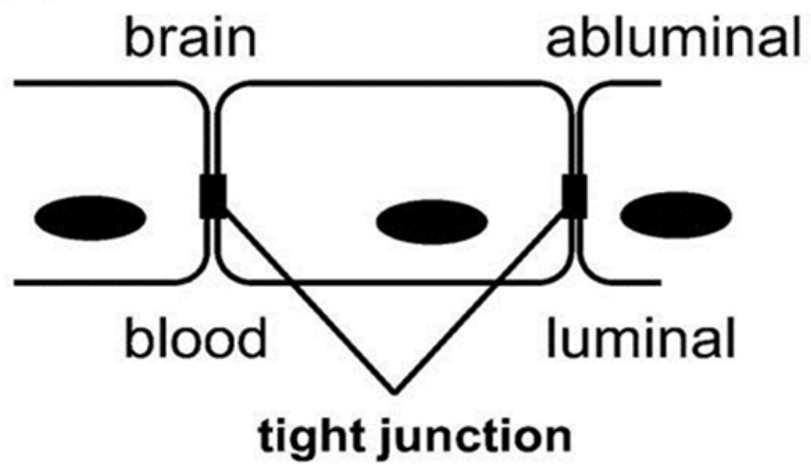

Figure 1-2. Schematic representation of the capillary endothelial cells that build up the BBB

A. Cross-section through a brain capillary.

B. Longitudinal section across the wall of the capillary, with the tight junctions that separate the membranes into the luminal (i.e. apical) and abluminal (i.e. basolateral) sides.

Source: Reprinted by permission from the American Physiological Society. Hagenbuch B et al. (2002) Transport of xenobiotics across the blood-brain barrier. News in Physiological Sciences 17:231-234. 
Terasaki and Hosoya, 1999). ABC transporters known to restrict CNS distribution of anti-cancer agents include ABCB1 [MDR1; P-glycoprotein (P-gp)], ABCC1 [multidrug resistance-associated protein 1 (MRP1)], $\mathrm{ABCC} 4$ (multidrug resistance-associated protein 4 (MRP4), and ABCG2 [breast cancer resistance protein (BCRP)] (PL and GM, 2003; Terasaki and Hosoya, 1999).

Another important family of transporters expressed on endothelial cells is the solute carrier (SLC) family of transporters (Koepsell and Endou, 2004). In contrast to the $\mathrm{ABC}$ transporter family, members of the SLC family do not require ATP for their function (Degorter et al., 2011; Hediger et al., 2004). SLC transporters mediate the movement of their substrates via several mechanisms, including carrier-mediated (i.e. facilitated diffusion), ion coupling, and ion exchange mechanisms (Hediger et al., 2004). They are primarily involved in the influx of drugs and endogenous compounds to the brain. Previous reports showed CNS expression of several SLC family members where they play an important role in drug transport across the BBB (Angeletti et al., 1997; Gao et al., 2000; Gao et al., 1999; Lin et al., 2010; Noe et al., 1997; Roberts et al., 2008; Urquhart and Kim, 2009; WM, 2003).

Another barrier involved in anti-cancer drug CNS distribution is the bloodcerebrospinal fluid barrier (BCSFB) (Johanson et al., 2005). This barrier is composed mainly of the choroid plexus, a layer of polarized epithelial cells lining the ventricles (Redzic and Segal, 2004). The choroid plexus is comprised of two layers, an inner layer of endothelial cells and an outer layer of ependymal cells (Redzic and Segal, 2004). While endothelial cells of the BCSFB are fenestrated, tight junctions are present between the outer ependymal cells (Redzic and Segal, 2004; Zheng et al., 2003). These tight junctions restrict the paracellular transport across the choroid plexus (Redzic and Segal, 2004; Zheng et al., 2003). Thus, to enter the CSF, molecules have to cross the fenestrated capillary wall and penetrate the ependymal cells of the choroid plexus. Similar to the $\mathrm{BBB}$, drug trafficking across the BCSFB is also controlled by efflux and uptake transporters (Kusuhara and Sugiyama, 2004).

Many factors are involved in the transport of drugs across the brain barrier membranes. Localization of efflux and uptake transporters either on the apical side of the BBB (facing the blood) or the basolateral side (facing the brain) will affect the disposition of their substrates in the brain extracellular fluid (ECF) (Figure 1-3) (Shen et al., 2009; Westerhout et al., 2011; Zhuang et al., 2006). Likewise, the localization of these transporters on the apical side of the choroid plexus epithelia (facing the CSF) or the basal side (facing the blood) will impact the translocation of their substrates in the CSF (de Lange, 2004; Shen et al., 2009; Wijnholds et al., 2000; Zhuang et al., 2006). In the following section, the localization of efflux transporters namely P-glycoprotein (P-gp, $\mathrm{ABCB} 1)$, breast cancer resistance protein (BCRP, $\mathrm{ABCG}$ ), and multidrug resistanceassociated protein 4 (MRP4) and their impact on the disposition of their substrates within different CNS compartments will be discussed. Furthermore, the expression of solute carrier transporters organic anion-transporting polypeptide 1A2 (OATP1A2), organic anion transporter 3 (Oat3), and the organic cation transporter 2 (OCT2) will be reviewed. 


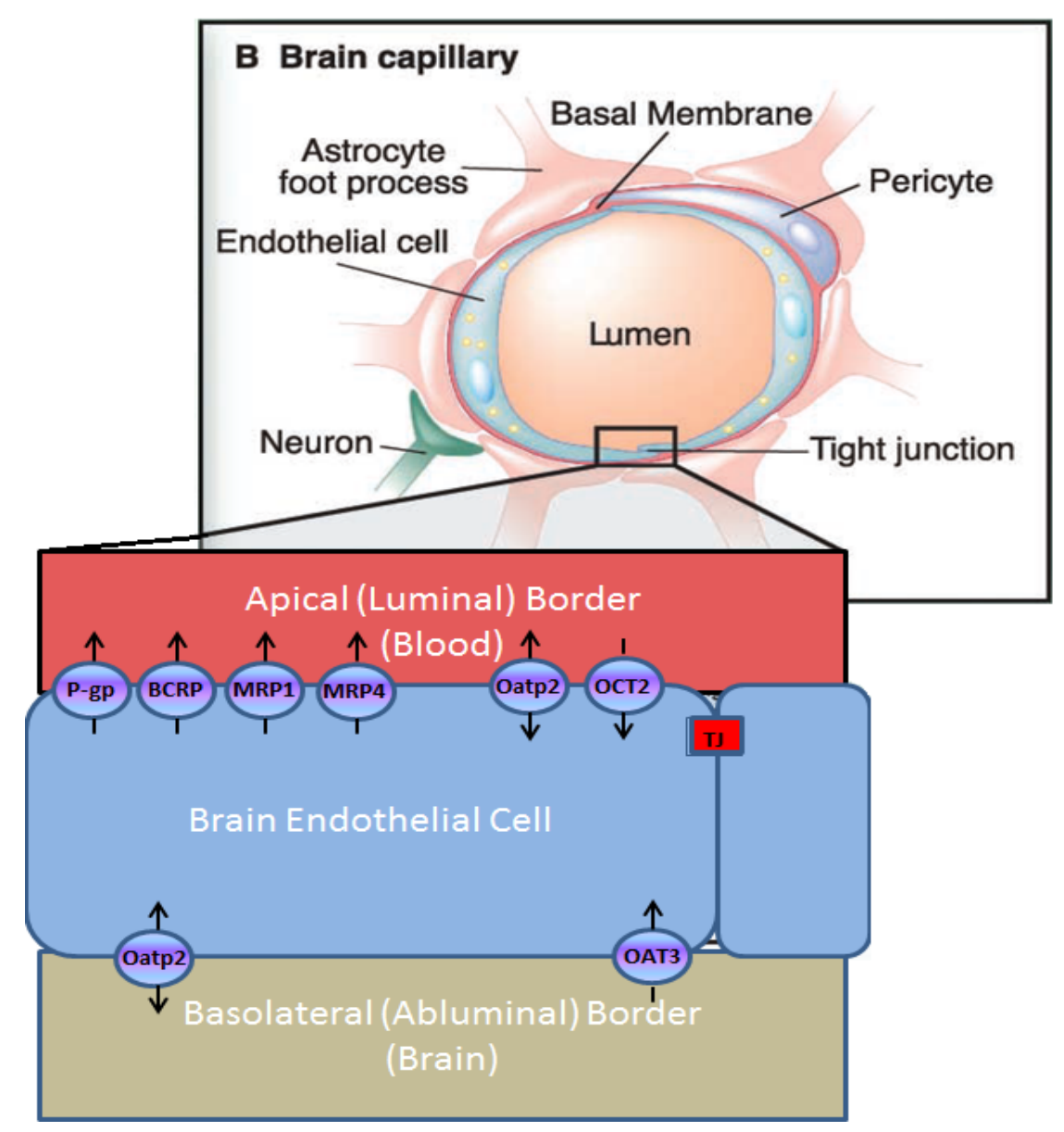

Figure 1-3. Schematic representation of the BBB

Brain endothelial cells are surrounded by a basal membrane, astrocyte foot processes, and supportive pericytes. Tight junctions in brain capillary endothelial cells prevent paracellular transport through the BBB. Transcellular diffusion through the BBB is affected by efflux and uptake transporters illustrated in the lower portion of the figure. P-gp, BCRP, MRP1, MRP4, and OCT2 are localized on the apical border while Oat3 is expressed predominantly on the basolateral membrane. Oatp2 is expressed at both apical and basolateral borders.

Source: Adapted by permission from the American Association for Cancer Research. Deeken J et al. (2007) The Blood-Brain Barrier and Cancer: Transporters, Treatment, and Trojan Horses. Clin Cancer Res 13:1663-1674. 


\subsubsection{ATP-Binding Cassette family}

\subsubsection{P-glycoprotein (P-gp/ABCB1)}

P-gp is the most widely studied of all efflux drug transporters. In humans, P-gp is encoded by the MDRl gene while two genes, $M d r l a$ and $M d r l b$, encode P-gp in the rodent brain (Bendayan et al., 2002; Gottesman, 1993; Schinkel et al., 1996). P-gp is highly expressed on the luminal side of the capillary endothelial cells of the BBB in humans, mice, rats, and dogs (Beaulieu et al., 1997; Cordon-Cardo et al., 1989; Hegmann et al., 1992; Thiebaut et al., 1989) (Figure 1-3). It is widely accepted that P-gp plays an important role in limiting the accumulation of its substrates in the brain ECF and thus conferring resistance to these agents (Nobmann et al., 2001; Pardridge, 2005; Schinkel et al., 1995).

Expression of P-gp at the ependymal cells of the choroid plexus has been established in humans, mice, and rats by several groups (Daood et al., 2008; Rao et al., 1999a; b; Zhuang et al., 2006). However, the exact localization of P-gp to either side of the choroid plexus is not clear yet. In humans, P-gp is thought to localize apically at the BBB (Beaulieu et al., 1997; Daood et al., 2008; Rao et al., 1999a). In rodents, P-gp was found to exhibit a granular expression pattern throughout the cytoplasm with enhanced expression on the apical side of the choroid plexus (Figure 1-4) (Zhuang et al., 2006). Given its localization, P-gp can play an important role in active transport of its substrates into the ventricular CSF. This will increase the concentration of P-gp substrates in the CSF. Using noninvasive single-photon-emission computed tomography, Rao et al showed that CSF levels of the P-gp substrate ${ }^{99 \mathrm{~m}}$ Tc-sestamibi is 100 -fold more than that in blood (Rao et al., 1999b). Our group showed that accumulation of topotecan, a P-gp substrate, was significantly higher in the CSF of wild-type mice compared to mice lacking P-gp, i.e. Mdrla/ $b^{-/-}$(Shen et al., 2009). Collectively, these data indicate an important role for P-gp in the blood to CSF transport of its substrates.

Important insights to the function of P-gp were obtained from experiments conducted with mouse models lacking either one or both of the P-gp encoding genes, i.e. Mdrla ${ }^{-/-}$and Mdrla/1 $b^{-/-}$mice (Schinkel et al., 1994; Schinkel et al., 1995; Wijnholds et al., 2000). These models were used to demonstrate the role of P-gp in drug disposition in the ECF or the CSF. Schinkel et al showed striking 12-fold higher brain concentrations of vinblastine, a P-gp substrate, in ECF of $M d r l a^{-/}$mice compared to wild-type mice (Schinkel et al., 1994). These models played an important role in studies that evaluate the role of P-gp in restricting CNS penetration of several molecularly targeted agents that are currently being tested for treatment of high-grade glioma (Agarwal et al., 2010; Marchetti et al., 2008; Poller et al., 2011). 


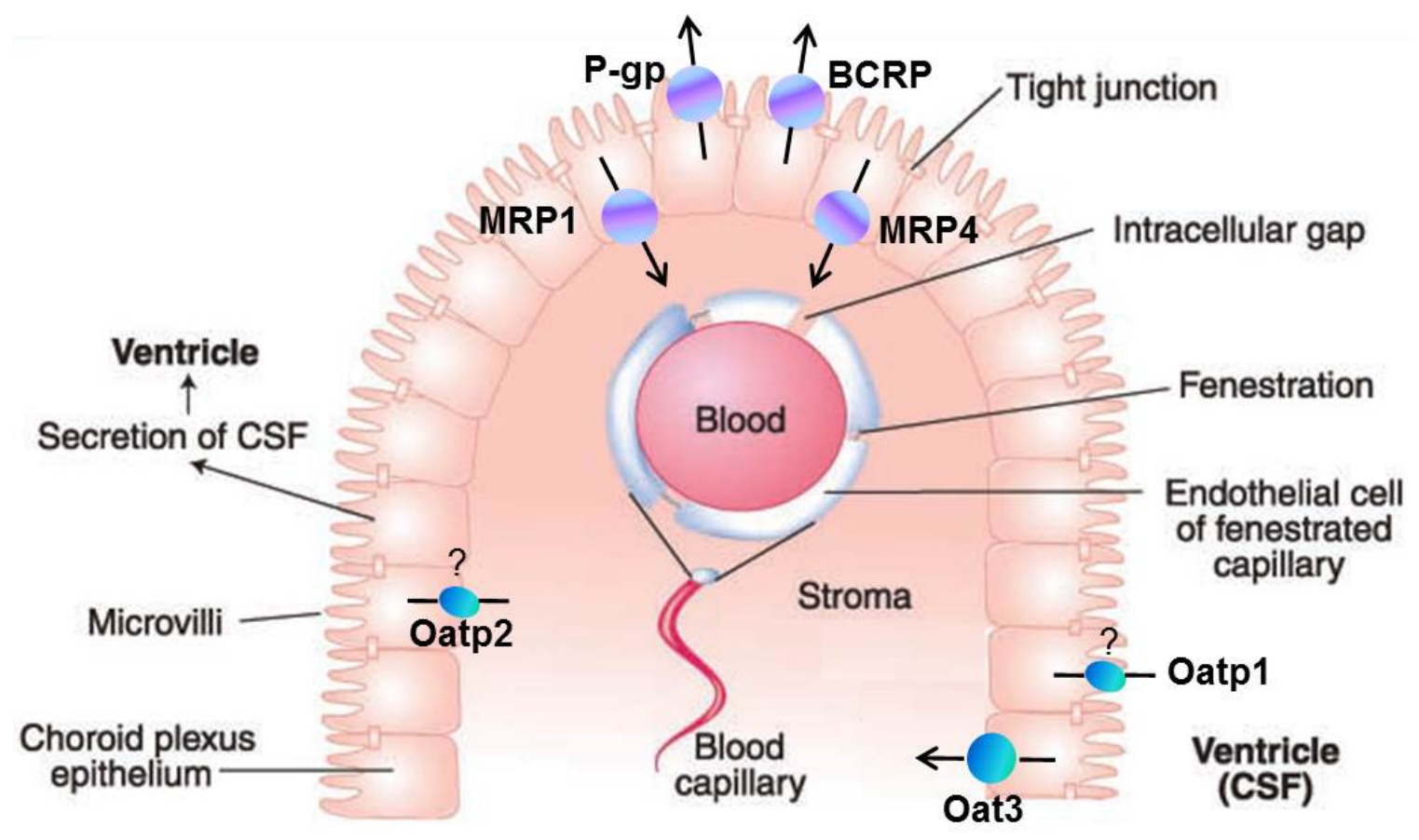

Figure 1-4. Schematic representation of the BCSFB

Fenestrated capillaries in the choroid plexus permit free transport of molecules. However, tight junctions on the choroid plexus epithelial cells limit paracellular transport.

The CSF-facing surface of the epithelial cells is called the apical border while that facing the blood side is called the basolateral border. The figure illustrates the current view of the localization of drug transporters at the choroid plexus epithelial cells. P-gp is predominantly located at the apical side and seems to transport into its substrates into the CSF. BCRP exhibits similar localization to P-gp. MRP1 and MRP4 are localized on the basolateral border and they are thought to mediate transport out of the CSF into the blood. Uptake transporters such Oat3 is predominantly expressed on the basolateral border. The rodent orthologues Oatp1 and Oatp2 corresponding to human OATP1A2, are expressed at the apical membrane of the choroid plexus in case of Oatp1 or at both apical and basolateral membranes.

Source: Adapted by permission from the American Association for Cancer Research. Deeken J et al. (2007) The Blood-Brain Barrier and Cancer: Transporters, Treatment, and Trojan Horses. Clin Cancer Res 13:1663-1674. 


\subsubsection{Multi-drug resistance associated proteins (MRPs/ABCC)}

The ABCC subfamily of ABC transporters consists of 12 members, nine of them (MRP1-9) have been implicated in conferring drug resistance. Of these nine members, MRP1 and MRP4 are the most relevant to anti-cancer drugs used in treatment of brain tumors.

MRP1 is expressed on the apical side of the capillary endothelial cells forming the BBB (Figure 1-3). According to this orientation, MRP1 can limit the penetration of their substrates to brain ECF. Mrp $1^{-/-}$mouse models show higher brain accumulation and enhanced sensitivity towards MRP1 substrates such as the topoisomerase II interacting agent, etoposide, compared to their wild type counterparts (Lorico et al., 1997; Wijnholds et al., 1997).

On the choroid plexus, Mrp1 is expressed on the basolateral side of the ependymal cells. This localization, in contrast to P-gp localization, allows the efflux of drugs from the CSF into the blood (Figure 1-4). The role of MRP1 in CSF to blood transport was confirmed by studies showing 10-fold higher etoposide accumulation in the CSF of $\mathrm{Mrpl}^{-/-}$mice (Wijnholds et al., 2000). Given its role on the BBB and the BCSFB, Mrp1 can serve as an important detoxification system of the whole brain (de Lange, 2004).

Similar to Mrp1, MRP4 is expressed on the apical membrane of the endothelial cells of the BBB (Figure 1-3) and on the basolateral membrane of the choroid plexus (Figure 1-4) (Huai-Yun et al., 1998; Leggas et al., 2004a; Nies et al., 2004; Roberts et al., 2008; Zhang et al., 2000). Leggas et al showed increased topotecan brain penetration in $\mathrm{Mrp}^{-/-}$mice compared to wild-type mice indicating a potential role for MRP4 in restricting topotecan brain ECF accumulation (Leggas et al., 2004a). Further, MRP4 was shown to reduce the intracellular accumulation of the tyrosine kinase inhibitor imatinib in MRP4 expressing Saos-2 cells (Hu et al., 2008). This suggests that MRP4 can play an important role in disposition of several chemotherapeutic and molecularly targeted agents. Further investigation is necessary to elucidate the role of MRP4 in brain transport of other molecularly targeted agents.

\subsubsection{Breast cancer resistance protein (BCRP/ABCG2)}

Another member of the $\mathrm{ABC}$ transporter family was first identified in a breast cancer cell line $\mathrm{MCF}-7 / \mathrm{AdrVp}$ and hence named breast cancer resistance protein (BCRP) (Doyle et al., 1998). Additional studies showed that BCRP is expressed on the apical side of the capillary endothelial cells in humans, rats, and mice brains where it has been implicated in brain to blood transport of its substrates (Figure 1-3) (Cooray et al., 2002; Hori et al., 2004). On the human choroid plexus, BCRP expression has not been extensively studied. However, studies from mouse and rat brain showed apical localization on the choroid plexus (Figure 1-4) (Zhuang et al., 2006). This P-gp-like

localization, suggests that BCRP limits the penetration of its substrates to brain ECF and 
transports these agents across the BBB into the systemic circulation. Similar to P-gp function on the BCSFB, BCRP was shown to transport its substrates into the CSF. In $B$ crp $1^{-/-}$mice, BCRP substrates showed lower CSF accumulation compared to wild-type mice (Shen et al., 2009; Zhuang et al., 2006).

BCRP and P-gp show a considerable overlap in their substrate specificity. Recent reports suggested that BCRP and P-gp cooperate to limit CNS penetration of their dual substrates (Agarwal et al., 2010; de Vries et al., 2007; Shen et al., 2009). Several tyrosine kinase inhibitors were shown to be dual substrates for P-gp and BCRP. For example, in $B c r p 1^{-/-}$mice or $M d r l a / b^{-/-}$mice, little to no difference was observed in brain penetration of several tyrosine kinases compared to wild-type mice. However, in $\mathrm{Mdrla} / \mathrm{b}^{-/ /} / \mathrm{Bcrpl} \mathrm{I}^{-/-}$ mice that lack both transporters, brain penetration of these agents are significantly elevated (Agarwal et al., 2011c; Chen et al., 2009; Polli et al., 2008). In some cases, drug levels in the brain are even higher than that in the systemic circulation which suggests a potential role of influx mechanisms responsible for the blood to brain transport of these molecules across the BBB (Agarwal et al., 2010; Kodaira et al., 2010; Kusuhara and Sugiyama, 2005).

\subsubsection{Pharmacological inhibition of efflux transporters}

Given the important effect of P-gp and BCRP on CNS anti-cancer drug disposition, several modulators of these transporters were developed in attempts to increase the delivery of anti-cancer drugs to therapeutically resistant tumors located behind the BBB. First generation inhibitors of P-gp were drugs known to be P-gp substrates such as verapamil and cyclosporin $\mathrm{A}$. The use of these agents in combination with anti-cancer agents was associated with significant drug-related side effects in patients (Sikic et al., 1997). The increased toxicity can be explained by first, the lack of specificity and poor binding affinity of these agents to P-gp. Thus, transport inhibition can only be achieved at high exposures that can lead to toxicity. Second, verapamil and cyclosporin A are inhibitors of cytochrome P450 enzymes, which are commonly responsible for the metabolism of anti-cancer agents. Thus, combining non-specific P-gp modulator with anti-cancer agents resulted in lower systemic clearance and consequently increased toxicity of anti-cancer agents.

Second generation modulators, such as the cyclosporin A analog, PSC833, are more specific and potent P-gp inhibitors. However, PSC833 also inhibited CYP3A enzymes. Therefore, third-generation inhibitors, such as elacridar (GF120918), and tariquidar (XR9576) (Hyafil et al., 1993; Roe et al., 1999), were developed to mainly modulate the transport function with little impact on liver metabolizing enzymes, which is reflected via better safety profiles in patients (Hyafil et al., 1993; Kruijtzer et al., 2002). Tariquidar is considered a specific P-gp inhibitor (Roe et al., 1999) while elacridar also inhibits human BCRP and mouse Bcrp1 (Hyafil et al., 1993; Maliepaard et al., 2001). To our knowledge, the efficacy of second or third generation transporter modulators in combination with molecularly targeted agents has not been assessed in patients with highgrade glioma. 


\subsubsection{Solute carrier transporters}

\subsubsection{Organic anion transporter-3 (OAT3)}

Oat3 is predominantly expressed on the basolateral side of mouse brain endothelial cells (Figure 1-3) (Ohtsuki et al., 2004). In rat brain, Oat3 is predominantly expressed on the basolateral side of the capillary endothelial cells with less expression on the luminal side (Kikuchi et al., 2003). This unique expression pattern enables Oat3 to mediate the transport of its substrates into or out of the brain. Reports studying CNS disposition of the typical Oat3 substrate p-aminohippurate (PAH) in rats showed both efflux and uptake processes of PAH to occur. However, the magnitude of PAH efflux was found to be 4 times higher than its uptake (Kusuhara et al., 1999; Kusuhara and Sugiyama, 2005). This suggests that Oat3 will have a net effect of extruding its substrates from the brain (Kusuhara and Sugiyama, 2005).

On the choroid plexus, Oat 3 is expressed on the basolateral border of the ependymal cells (Figure 1-4). Oat3 seems to mediate the transport of its substrates in an opposite direction to that of P-gp and BCRP (i.e., from the CSF to the blood) (Kikuchi et al., 2003; Sykes et al., 2004).

Oat3 has been shown to transport several drugs with antitumor activity such as 6-mercaptopurine and 6-thioguanine (Mori et al., 2004). However, further studies are warranted to assess the contribution of Oat 3 to the disposition of anti-cancer agents used to treat glioma.

\subsubsection{Organic cation transporter-2 (OCT2)}

OCT2 is primarily expressed at the basolateral membrane of renal proximal tubules (Karbach et al., 2000; Motohashi et al., 2002) but it has also been detected in human neurons (Slitt et al., 2002). OCT2 is expressed on the apical side of human brain endothelial cells similar to the rodent orthologues, Oct1 and Oct2 (Figure 1-3) (Lin et al., 2010). Typical OCT2 substrates are organic cations or weak bases (Nies et al., 2011). However, uncharged compounds such as cimetidine and non-ionizable compounds such as corticosteroids were shown to interact with OCT2 in a pH-dependent manner (Barendt and Wright, 2002). Further, OCT2 was shown to interact with organic anions such as probenecid (Hsyu et al., 1988). This substrate poly-specificity can be attributed to the existence of distinct hydrophobic and cationic sides on OCT2 (Rennick, 1981).

On the BBB, OCT2 mediates the facilitated diffusion of several endogenous substrates and drugs. For example, the administration of the OCT2 inhibitor, amantadine, reduced the uptake of the OCT2 substrate, N-methyl-4-phenyl-1,2,3,6-tetrahydropyridine in brain ECF (Lin et al., 2010). On the BCSFB level, the role of OCT2 is not clear yet. OCT2 mRNA expression could not be detected on the rat choroid plexus epithelium which suggests minimal, if any, contribution of OCT2 to drug disposition across the 
BCSFB (Choudhuri et al., 2003). Further studies are needed to understand the role of OCT2 in CNS disposition of several anti-cancer agents. The availability of $\mathrm{Oct} 2^{-/-}$murine models will allow better understanding of the role of this transporter in carrier-mediated transport of several anti-cancer agents (Jonker et al., 2003).

\subsubsection{Organic anion transporter polypeptide 1A2 (OATP1A2)}

OATP1A2 is a member of the SLCO family of influx transporters that regulates the cellular level of several endogenous and exogenous compounds. OATP1A2 is expressed on the apical membrane of the brain endothelial cells. The expression of human OATP1A2 on the choroid plexus cells remains to be clarified. In rodents, two orthologues correspond to the human OATP1A2, Oatp1 and Oatp2. Oatp2 is highly expressed at both apical and basolateral membranes of the rat brain endothelial cells (Figure 1-3) while its localization in the choroid plexus is restricted to the basolateral membrane (Figure 1-4) (Angeletti et al., 1997; Gao et al., 1999). Oatp1 is not expressed at the BBB but its expression has been detected at the apical membrane of the choroid plexus (Figure 1-4) (Angeletti et al., 1997; Gao et al., 1999).

Given the high expression levels of OATP1A2 in brain tissue and its broad spectrum of substrates, it is likely that this transporter play an important role in drug CNS disposition (Urquhart and Kim, 2009). For example, OATP1A2 was shown to play an important role in the transport of its substrates of opioid peptides across the BBB (Gao et al., 2000). Further, brain accumulation of several OATP1A2 substrates was reduced in mouse model lacking Oatp2 (Ose et al., 2010). Interestingly, several OATP1A2 substrates, such as levofloxacin and methotrexate, are associated with severe CNS toxicity and thus OATP1A2-mediated transport may partially explain the CNS associated side effects with these agents (Maeda et al., 2007). In vitro, OATP1A2 has been shown to increase imatinib intracellular accumulation (Hu et al., 2008). Further investigation is warranted to elucidate the role of this transporter in disposition of other tyrosine kinase inhibitors.

\subsection{ANTI-CANCER DRUG PENETRATION IN HIGH-GRADE GLIOMAS}

\subsubsection{The blood-tumor barrier in malignant glioma}

In contrast to the tightly regulated $\mathrm{BBB}$ in the normal brain, the $\mathrm{BBB}$ in highgrade gliomas is structurally and functionally abnormal (Blakeley et al., 2009; Gerstner and Fine, 2007; Miyagami and Katayama, 2005). Abnormal features develop in response to the imbalance between the high demand of oxygen and nutrients by the rapidly proliferating tumor cells and the poor supply capacity of the existing blood vessels. Such imbalance, coupled with poor tumor lymphatic drainage, leads to a harsh tumor microenvironment characterized by hypoxia, decreased nutrients, and accumulation of metabolic wastes (Bergers and Benjamin, 2003; Fukumura and Jain, 2007; Jain, 2005; 
Padera et al., 2002). Tumor cells overcome these unfavorable conditions by upregulating several proangiogenic factors such as VEGF (Ferrara, 2004), PDGF (Battegay et al., 1994), and basic fibroblast growth factor (bFGF) (Friesel and Maciag, 1995). All these factors induce endothelial cell survival, proliferation, and migration by direct interaction with their receptors expressed on endothelial cells (Ucuzian et al., 2010). Upregulation of these proangiogenic factors initiates the development of neovasculature through the process of angiogenesis (Bergers and Benjamin, 2003; Jain et al., 2007).

The newly developed tumor vessels represent a major determinant of glioma microenvironment and the principle means for anti-cancer drug delivery to the tumor. Therefore, the structural and functional properties of these vessels will significantly influence the concentration and distribution of anti-cancer agents throughout the tumor (Blakeley et al., 2009; Jain, 2005).

While normal vessels consist of an organized network of venules, capillaries, and arterioles, tumor vessels are characterized by their heterogeneous distribution. Electron microscopic analysis of tumor endothelial cells in malignant gliomas indicated that they are composed of immature capillaries (Miyagami and Katayama, 2005). Structurally, these vessels are tortuous and dilated with abnormal basement membranes (Brown and Wilson, 2004; Carmeliet and Jain, 2000). Furthermore, tumor vessels lack direct physical contact with pericytes, which in normal vasculature are known to stabilize the endothelial cells and regulate their homeostasis and maturation (Figure 1-3) (Eberhard et al., 2000; Gerhardt and Betsholtz, 2003). Moreover, tumor vessels experience continuous compression from the rapidly dividing tumor cells (Padera et al., 2004).

These structural defects render tumor vessels leaky with higher permeability when compared to normal vessels (Dvorak et al., 1995; Dvorak et al., 1999). The leakiness of tumor vessels will lead to extravasation of plasma components such as fibrin and albumin to brain ECF leading to increased interstitial fluid pressure, which is exacerbated by inefficient tumoral lymphatic drainage (Fukumura and Jain, 2007; Padera et al., 2002). The high interstitial fluid pressure results in tumor associated edema, which is a universal observation in gliomas and responsible for many of the disease associated symptoms (Fukumura and Jain, 2007; Papadopoulos et al., 2004).

Because of the aforementioned hyperpermeablity, it is expected that anti-cancer drugs can accumulate more easily in tumor ECF. This may explain the high anti-cancer drug penetration reported in several preclinical and clinical trials of high-grade gliomas where drug accumulation in tumors was generally close, or sometimes higher, to plasma concentrations indicating a breached BBB. For example, Hofer et al reported high gefitinib concentrations ( $>10$-fold of the plasma concentrations) in adult GBM patients (Hofer and Frei, 2007; Hofer et al., 2006). Several clinical and preclinical studies also reported similar results for other drugs such as methotrexate (Blakeley et al., 2009), temozolomide (Zhou and Gallo, 2009), topotecan (Carcaboso et al., 2010), and vincristine (Wang et al., 2010). 
On the other hand, other studies considered poor CNS penetration of several chemotherapeutics to be a significant factor in the failure of these regimens in treatment of malignant glioma (Doolittle et al., 2007; Lassman et al., 2005; Motl et al., 2006b; Muldoon et al., 2007), suggesting that drug tumoral penetration is not solely dependent on the hyperpermeability of the endothelial cells within the tumor microenvironment but also depends on the physicochemical properties of the drug.

It is also important to note that only the vessels at the tumor core are considered hyper-permeable and leaky. The leading tumor edge that invades through brain parenchyma is thought to make use of the existing normal brain vasculature with a relatively intact BBB. These intact endothelial cells will limit drug accumulation at the tumor edge (Fine et al., 2006; Gilbertson and Rich, 2007; Pitz et al., 2011). Thus, anticancer drug concentrations are expected to be higher at the core than at the invasive proliferative edge of the tumor (Gerstner and Fine, 2007; Pitz et al., 2011). This concept was supported by a clinical trial assessing paclitaxel concentrations at the tumor core, tumor periphery, and at the tumor-normal brain interface in high-grade glioma patients. Highest levels of paclitaxel were found in the tumor core, followed by the tumor periphery, with lowest paclitaxel concentrations found in the normal surrounding brain tissue (Fine et al., 2006; Gerstner and Fine, 2007). Improving the delivery of anti-cancer drugs to the clinically challenging infiltrative tumor cells may improve treatment outcome of high-grade glioma as these invasive cells represent a clinical challenge and are considered an important determinant for tumor recurrence (Giese et al., 2003).

\subsubsection{The glioma cell barrier}

High-grade glioma is composed of three distinct, yet interrelated, compartments. First, a vascular compartment formed of both existing blood vessels from normal brain tissue and newly developed tumor vasculature. The second is the ECF compartment, and the third is the intracellular compartment where most drugs currently used in treatment of high grade gliomas exert their anti-tumor effect (Baselga, 2006; Hammarlund-Udenaes, 2010; Hammarlund-Udenaes et al., 2008; Reichel, 2009). Though this three-compartment concept may not reflect the pathological complexity of the tumor, it provides a useful platform to understand drug trafficking mechanisms within different tumor compartments.

Anti-cancer drug disposition within these three compartments is determined by intricate microenvironmental properties of each compartment. Drug diffusion from the vascular to the ECF compartments is controlled by the leakiness of the vessels as well as the high interstitial fluid pressure. On one hand, anti-cancer agents may diffuse from the leaky tumor vessels and accumulate in tumor ECF compartment (Blakeley et al., 2009; Carcaboso et al., 2010; Hofer and Frei, 2007; Hofer et al., 2006; Zhou and Gallo, 2009). On the other hand, the lack of pressure difference between the vascular and interstitial fluid compartments, as a result of the high interstitial fluid pressure, may lead to a stagnant blood flow which will limit drug accumulation in tumor ECF (Fukumura and Jain, 2007). 
Several studies assessing anti-cancer drug penetration at the tumor core of highgrade glioma ECF show high tumor penetration ratio relative to plasma concentrations (Blakeley et al., 2009; Carcaboso et al., 2010). This suggests that anti-cancer agents are capable of reaching the tumor ECF. Determining whether the anti-cancer drug concentration achieved within the tumor is therapeutically effective requires determining the unbound drug concentrations at the active site. Most anti-cancer agents currently used for treatment of high-grade glioma exert their anti-tumor effect by interacting with intracellular targets. Even drugs that target receptor tyrosine kinases expressed on tumor cell membranes often interact with cytoplasmic domains of their targets and thus require intracellular accumulation of these agents (reviewed in Baselga, 2006).

Efflux transporters expressed on glioma cell membranes restrict intracellular accumulation of anti-cancer drugs and therefore provides an additional barrier (Agarwal et al., 2011b; Lee et al., 2001). Several reports showed the expression of P-gp (Calatozzolo et al., 2005; Decleves et al., 2002; Demeule et al., 2001; Fattori et al., 2007; Nabors et al., 1991), BCRP (Carcaboso et al., 2010; Decleves et al., 2006), as well as MRPs 1 and 4 (Bronger et al., 2005; Calatozzolo et al., 2005; Decleves et al., 2002; Mohri et al., 2000) in cases of malignant glioma.

Due to the effect of efflux transporters on glioma cell membrane, anti-cancer drugs may accumulate in the ECF compartment while only a fraction of the drug can accumulate inside the tumor cell (Carcaboso et al., 2010). Furthermore, only a fraction of the drug that reaches the intracellular compartment will be free to interact with its target while the rest remains nonspecifically bound to intracellular lipids and proteins (Hammarlund-Udenaes et al., 2008; Reichel, 2009). This will further reduce the amount of drug available to exert anti-tumor effect (Hammarlund-Udenaes, 2010; HammarlundUdenaes et al., 2008; Maurer et al., 2005; Reichel, 2009). Unfortunately, very little information is known about intracellular drug accumulation due to the difficulty of sampling the intracellular compartment in vivo. Thus, determining whether therapeutically effective concentrations can be achieved at the target site cannot be directly assessed in vivo using currently available methodologies (Hammarlund-Udenaes et al., 2008; Reichel, 2009).

Most of the studies assessing anti-cancer tumoral penetration rely on brain homogenization techniques. Several of these studies reported high tumor penetration profiles of anti-cancer drugs relative to plasma concentrations (e.g., gefitinib tumor concentrations reported to be more than 10-fold higher than that of the plasma concentrations) (Hofer and Frei, 2007). The techniques applied in these studies do not discern between any of the three tumor compartments. They also do not account for binding of the drug to proteins either in plasma or in tumor tissue. Therefore, no conclusions can be made regarding whether the achieved concentrations of the unbound drug at the target site are effective or not.

A study conducted by our group assessing intracellular topotecan distribution by analyzing both total and ECF concentrations in an orthotopic xenograft mouse model of glioma showed an inverse correlation between ECF concentrations and intracellular 
distribution (i.e., higher ECF concentrations correlate with lower intracellular distribution). Inhibiting efflux transporters, specifically P-gp and BCRP, at the tumor cell membrane decreased topotecan ECF concentrations and increased its intracellular distribution, indicating a critical role of efflux transporters in limiting anti-cancer drug access to the intracellular compartment (Carcaboso et al., 2010).

The complexity of assessing anti-cancer drug penetration in different tumoral compartments underscores the importance of understanding the different methods used to sample each compartment as well as the need for clinically relevant animal models that recapitulate features of the human disease. In the next two sections, animal models of high grade glioma currently available to study anti-cancer drug penetration and the approaches used to evaluate drug penetration within the tumor will be discussed.

\subsection{MURINE MODELS OF MALIGNANT GLIOMA}

The need for clinically relevant murine models of high-grade glioma is compelling. Such models will allow researchers to translate their findings from preclinical experiments to the bedside. The ideal murine model for studying anti-cancer drug accumulation in glial tumors should accurately recapitulate the histological features of high-grade glioma including hypoxia, intense vascular proliferation, and the invasive growth pattern of these tumors. This model should also reflect the interaction between the tumor and host stroma. Tumors in such an ideal model should have high penetrance and a reasonable latency that allows growth of tumors within adequate period that reflects, even partially, the human disease while still providing a window for testing of anti-cancer agents before the animals become moribund. Finally, such a model should mimic the genetic profile of high-grade glioma observed in patients.

Despite the availability of several murine models of malignant glioma, an ideal model that recapitulates all aspects of the disease does not exist. Every model provides a set of advantages and disadvantages to the research question. Depending on the purpose of the experiment, investigators should weigh the pros and cons of the models available and decide which model provides an adequate recapitulation of important effectors regarding their experiment. In this section, the pros and cons of some of the available models will be discussed with emphasis on their relevance to glioma histology, tumor microenvironment, genetic representation, and their suitability for drug penetration studies.

\subsubsection{Subcutaneous xenograft models}

Due to their relatively short latency, ease of establishment, predictable growth pattern, and the simplicity of monitoring tumor growth without the need for complex imaging techniques, subcutaneously implanted xenograft models are widely used for monitoring tumor response to chemotherapeutic regimens. Tumors implanted in these models commonly display features of hypoxia (Gillespie et al., 2007) and the 
development of neovasculature (Kim et al., 1993; Saleh et al., 1996). However, subcutaneous tumors fail to provide adequate resemblance to the microenvironment or growth pattern of intracranial glioma tumors in humans. For example, subcutaneously implanted models do not acquire the invasive growth characteristics of high-grade gliomas and grow mainly as a "bag" of tumor cells, bearing no resemblance to glial tumors (Antunes et al., 2000).

Further, the gene expression profiles of glioma cell lines implanted subcutaneously in nude mice was significantly different from intracranial tumors established from the same cell lines (Camphausen et al., 2005a; b). This indicates that subcutaneous growth of subcutaneous tumors provides different and probably irrelevant in vivo growth conditions (Camphausen et al., 2005a; b).

The use of these models to determine drug tumoral penetration is inadequate as these models neither recapitulate the degree of high-grade glioma microvascular proliferation nor the different CNS barriers against drug penetration (de Vries et al., 2009). This is supported by reports showing different penetration profiles of anti-cancer drugs in subcutaneous tumors when compared to their orthotopic counterparts (Ma et al., 2003). Consequently, anti-cancer drug penetration studies conducted using subcutaneous xenograft models are not indicative of the intracranial tumor situation and thus these models should not be used to study CNS drug penetration.

\subsubsection{Orthotopic xenograft models}

The relative ease of engraftment, rapid tumor growth, and short latency from tumor inoculation until morbidity have made orthotopic xenograft models an essential tool for in vivo efficacy studies of anti-cancer drugs in the past decade. These models are considered more appropriate than subcutaneous xenograft models in predicting drug efficacy and CNS drug penetration. However, concerns have been expressed regarding the clinical relevance of the tumor microenvironment in these models and whether they represent accurate models to study anti-cancer drug penetration to glial tumors.

Although some orthotopic xenograft models provide adequate recapitulation of the histological and invasive properties of malignant gliomas (e.g., U251) (Houchens et al., 1983; Radaelli et al., 2009), many commonly used models fail to recapitulate any of these properties (Becher and Holland, 2006; Jacobs et al., 2011). For example, upon intracranial inoculation of U87-MG cells in immune compromised mice, huge circumscribed tumors with clear tumor edge separating the tumor tissue from the surrounding normal brain are formed (Carcaboso et al., 2010; de Vries et al., 2009; Radaelli et al., 2009). As mentioned earlier, this growth pattern is vastly different from that of infiltrative tumors observed in patients (Giese et al., 2003). Unfortunately, U87-MG cell line has been one of the most frequently used cell lines for xenograft studies. This cell line has significantly contributed, perhaps in a biased way, to our understanding of high-grade gliomas. 
Another pitfall of xenograft models in general is their reliance on in vitro culturing of tumor cells. Cells are cultured on plastic plates or flasks may acquire or lose novel genetic properties (Camphausen et al., 2005a; b; Kucherlapati, 2012). For example, GBM cell lines cultured in vitro cannot preserve EGFR focal amplification, a common genetic alteration in $\sim 50 \%$ of adult patients with GBM (Pandita et al., 2004). Attempts to preserve the genetic properties of the original tumor included direct implantation of tumors from patients in immune-compromised mice (Pandita et al., 2004). Other trials focused on in vitro culturing of primary tumor cells under serum-free neurobasal media supplemented with different concentrations of growth factors such as EGF and bFGF instead of serum-based culture conditions (Lee et al., 2006; Schulte et al., 2012). Further studies are required to demonstrate the benefit of these approaches on recapitulating the features of the original tumor (Schulte et al., 2012).

Importantly, a tumor implanted in an immune compromised host lacks the contribution of the host-tumor interaction, which may impact the tumor propensity to invade, metastasize, and develop new blood vessels (Becher and Holland, 2006). This may explain the inability of several orthotopic glioma models to recapitulate many of the histological features of HGGs including the invasiveness and the intense microvascular proliferation.

Orthotopic xenograft models are used, almost exclusively, for drug penetration studies in high-grade gliomas as they provide some depiction of the different CNS barriers (Carcaboso et al., 2010; Ma et al., 2003). Some inherent limitations of these models are unlikely to be avoided such as the genetic properties acquired on culture and the inability to recapitulate the host-tumor interaction. However, adequate orthotopic models for drug penetration studies should recapitulate tumor invasiveness and microvascular proliferation observed in human tumors. In general, the data obtained using these models should be interpreted in light of the limitations discussed earlier.

\subsubsection{Genetically engineered mouse models}

Given all the aforementioned shortcomings of glioma xenograft models together with the advancements in gene targeting technologies, more focus has been directed to develop genetically engineered mouse models (GEMMs) that can represent the genetic profile and histological features of high-grade glioma including the invasive growth pattern of these tumors. In general, these models reproduce the microenvironment of high-grade glial tumors better than xenograft models (Becher and Holland, 2006; Chow and Baker, 2012; Kucherlapati, 2012).

GEMMs can be categorized into germ-line and somatic GEMMs. In germ-line GEMMs, genetic alterations are introduced in the embryonic stage and is maintained through breeding (Aguzzi et al., 1994; Heyer et al., 2010). Germ-line GEMMs are beyond the scope of our discussion. The reader is directed to several excellent research articles and reviews where germ-line GEMMs have been discussed in more detail (de 
Vries et al., 2009; Ding et al., 2001; Reilly et al., 2000; Wang et al., 2009; Xiao et al., 2002; Zhu et al., 2005).

Somatic GEMMs harbor genetic alterations in some of its somatic cells but not in the germ line cells. Of these, conditional GEMMs enable the induction of the genetic event in a temporally and spatially controlled manner (i.e., the genetic alteration can be induced at a certain time during the mouse development and in a certain subset of cells (Gossen and Bujard, 1992; Heyer et al., 2010). In this section, we will highlight some of the recently reported somatic GEMMs that may provide reasonable tools for studying anti-cancer drug penetration in high-grade gliomas.

Marumoto and colleagues reported a novel GEMM that recapitulates most features of high-grade gliomas (Marumoto et al., 2009). Tumors develop upon stereotactic injection of lentiviral vectors expressing H-RasV12 and AKT oncogenes in immune compromised mice with GFAP-Cre/ $p 53^{+/-}$background that restricts the expression of H-RasV12 and AKT oncogenes to GFAP (glial fibrillary acidic protein) expressing cells, i.e. astrocytes and neural progenitor cells. Tumors can be established in different brain locations with variable degree of penetrance (hippocampus 100\%, subventricular zone 75\%, and the cortex 7\%) (Marumoto et al., 2009).

The genetic drivers of this model are different from that observed in patients. While RTK/PI3K pathway is frequently deregulated in gliomas (Guha et al., 1997; Pollack et al.), Ras mutation and AKT amplification are relatively rare events in highgrade gliomas ( $2 \%$ for each) (TCGA, 2008). Other concerns noted about the viral delivery approach include unpredictable delivery and efficiency which may result in different cell groups transduced with either one of these oncogenes (Ras and AKT) or both leading to multiple tumor types in the same mouse (de Vries et al., 2009; Heyer et al., 2010). Finally, the contribution of the host immune response to tumor growth is lacking in this model as tumors are generated in immune compromised mice (Marumoto et al., 2009).

Tumors of this model showed many of the histological features of high-grade gliomas including high cellularity, necrosis, invasiveness through brain parenchyma, and a high degree of vascular proliferation. Reasonable latency ( $\sim 3$ months) was observed for the development of these tumors. These properties render this model a reasonable model to study anti-cancer drug penetration in glioma (Marumoto et al., 2009).

Another recently reported model involves intracranial injection of GFAP-Cre or CMV-Cre in compound LoxP-conditional mice with p53 or pten deletions (de Vries et al., 2010). Viral transduction of Cre leads to K-RasV12 expression and deletion of the tumor suppressor gene Ink4a/Arf, resulting in high-grade astrocytoma with $100 \%$ penetrance. These tumors demonstrate most of the histological features of high-grade glioma tumors with increased mitosis, nuclear atypia, giant cell formation, and pseudopalisading necrosis. Tumor vessels of this model express BCRP, a feature observed in vessels from human glioblastoma (Zhang et al., 2003). Since these tumors are luciferase-labeled, their growth can be easily monitored using bioluminescence. 
As noted in the previous model, Ras mutations are rare genetic events in glioma and thus the genetics of this model might not be representative of the human disease. However, this model presents a useful tool which combines the relative ease of induction, simple monitoring of tumor growth, and retaining important markers of tumor vasculature (de Vries et al., 2010). Unfortunately, this model did not show glioma-like angiogenic features and thus lacks an important determinant of tumoral drug penetration in high-grade glioma.

Another tumor model to induce conditional loss of function is the inducible $\mathrm{CreER}^{\mathrm{TM}}$-loxP system, which allows temporal and spatial control of somatic deletion of tumor suppressor genes (Gossen and Bujard, 1992; Jonkers and Berns, 2002). Cre recombinase is expressed under the control of the promoter of a site-specific marker (e.g., GFAP in case of astrocytes and neural precursors). Administering the inducer (e.g., tamoxifen) will result in nuclear translocation of the Cre recombinase, which induces somatic deletion of "floxed" tumor suppressor genes (Metzger and Feil, 1999; Pluck, 1996).

Few glioma models using this system have been reported. For example, Liaguno and colleagues showed that deletion of tumor suppressor genes $N f 1, p 53$, and/or Pten in Nestin-expressing cells lead to development of high-grade gliomas (Alcantara Llaguno et al., 2009). Tumors of this model showed all of the typical histological features of highgrade gliomas within $\sim 4$ months of induction with $100 \%$ penetrance. Finally, Chow et al used CreER ${ }^{\mathrm{TM}}$-loxP system to show that deletion of $p 53$, Pten, and $R b$ results in initiation of high-grade astrocytoma with high penetrance ( 85\%) (Chow and Baker, 2012; Chow et al., 2011).

Both CreER ${ }^{\mathrm{TM}}$-loxP dependent models discussed above bestow many of the genetic and histological features of malignant glioma. Another point to note is the heterogeneity associated with the tumor location, histology, and the secondary spontaneous mutations associated with these models (Alcantara Llaguno et al., 2009; Chow et al., 2011). While this feature provides better recapitulation of the heterogeneous nature of malignant glioma, it adds more complexity to conducting preclinical experiments and interpreting the results. Other pitfalls of these models are the relatively long latency of tumor growth ( 4-6 months) and the relative difficulty of monitoring in situ tumor growth. However, with better experimental design in monitoring tumor growth and location, the use of novel imaging modalities such as MRI, and careful scheduling of experiments before animals succumb to disease, these models can provide an attractive tool to study anti-cancer drug penetration to high-grade glioma.

\subsection{APPROACHES TO EVALUATE ANTI-CANCER DRUG PENETRATION IN BRAIN TUMORS}

The extent of drug penetration within brain tumors can be calculated as the ratio of unbound drug concentrations in the tumor relative to unbound concentrations in the plasma at steady state, or by the ratio of unbound area under the curve (AUC) in tumor 
relative to unbound plasma AUC. Different sampling strategies and pharmacokinetic approaches have been used to determine tumoral drug penetration. These methods differ in the number of samples that can be obtained, their ability to provide compartmentspecific information about drug penetration, and their ability to account for drug protein binding. In this section, the pros and cons of these methods will be briefly discussed.

\subsubsection{Whole tissue homogenization}

Due to the ease of sample collection and processing, most preclinical and clinical studies have relied on homogenizing whole brain or tumor samples to study anti-cancer drug concentration expressed as the amount of drug per gram tissue (e.g., ng/gm tissue) (Hofer and Frei, 2007; Hofer et al., 2006; Lassman et al., 2005; Zhou and Gallo, 2009). In preclinical studies, this method involves sacrificing 3-6 animals per time point and collecting blood and tissue at each time point. Therefore, large numbers of animals are required, typically around 25-40 animals per study (Zhou and Gallo, 2009). In clinical studies, tumor tissue can be collected only during surgical removal of the tumor and thus only one time point can be studied in each patient.

This method does not discern between different tumor compartments (e.g., vascular, ECF, and intracellular), and consequently fails to provide information about anti-cancer drug concentration within any specific compartment. This method considers the tumor one homogenous entity and thus provides the total drug concentration in all tumor compartments.

Another limitation to tumor homogenization is that it does not account for drug binding to the tumor tissue. Only unbound drug can pass through the barriers, enter tumor cells, bind to its target and exert the anti-tumor effect (de Lange and Danhof, 2002; Smith et al., 2010). High total drug concentration in the tumor $\left(\mathrm{A}_{\text {tumor }}\right)$ can be misleading, as it does not necessarily reflect high pharmacologically available concentrations at the active site. In other words, $A_{\text {tumor }}$ may reflect drug in the vascular compartment, ECF compartment, or even drug bound to nonspecific, off target intracellular proteins. It is also important to mention that binding of the drug in brain or tumor is different from that in plasma due to different protein and lipid contents between plasma and brain (Richmond, 1985).

\subsubsection{CSF Sampling}

CSF sampling is an alternative method to study CNS and tumor drug disposition (Shen et al., 2004). This method provides information on the unbound drug concentrations (Lin, 2008). As discussed earlier, CSF is separated from the blood via the BCSFB, which is mainly dependent on efflux transporters expressed at either apical or abluminal sides of the choroid plexus (Motl et al., 2006a). CSF sampling is commonly obtained through a procedure known as lumbar puncture which usually provides a single

CSF sample (Hill et al., 1999). However, for serial sampling a cannula should be inserted 
in the cisterna magna (Bruce and Oldfield, 1988; Schumann and Crisman, 1985). Given the ease of obtaining serial samples from this method, several preclinical and clinical studies relied on CSF sampling as a surrogate for brain or tumor concentrations (Blaney et al., 1998; Broniscer et al., 2007b; Vanbree et al., 1989; Vistelle et al., 1994).

However, evidence from several reports suggests that CSF concentrations are poor surrogates of brain ECF concentrations (de Lange and Danhof, 2002; Lin, 2008). For example, several P-gp substrates exhibit different penetration profiles in brain ECF compared to CSF (Shen et al., 2009). This can be explained by P-gp localization at the BBB and the BCSFB, which allows P-gp to transport its substrates from brain ECF to blood at the BBB and into the ventricular CSF at the BCSFB. Similar reports showing different CSF and ECF penetration of several other drugs are available (e.g. morphine, nelfinavir, and antipyrine) (Kaddoumi et al., 2007; Mayer et al., 1959; Stain-Texier et al., 1999). Further, the increased BBB permeability observed at the core of high-grade glioma tumors is likely to cause different penetration profiles in tumor ECF than in CSF, which means that CSF concentrations cannot be used as a surrogate to estimate tumor ECF concentrations (Stain-Texier et al., 1999).

\subsubsection{Cerebral microdialysis}

Cerebral microdialysis is a sampling technique that involves the insertion of a semi-permeable probe into the target tissue (e.g., brain or tumor). This probe is then perfused by a solution (i.e. perfusate) that mimics the composition of the target tissue (e.g., ECF or CSF). Molecules (e.g., anti-cancer drug) in the target tissue will diffuse through the semi-permeable membrane according to their concentration gradient. Finally, the collected sample (i.e. dialysate) is analyzed using analytical methods sensitive enough to detect the drug in the small sample volume collected.

Microdialysis provides a useful tool to sample a distinct tissue compartment, the ECF or ventricular CSF. An important advantage of this technique is that only unbound drug can pass through the pores of the semi-permeable membrane given the low molecular weight cutoff of the probe $(\sim 5 \mathrm{kDa})$ (Tsai, 2003). Therefore, inserting a microdialysis probe in the tumor can allow investigators to determine the unbound (active) drug concentration in the ECF (Tsai, 2003). It also allows serial sampling from a single animal, thus reducing the number of animals required for preclinical studies (de Lange et al., 1997; Hammarlund-Udenaes et al., 1997).

Microdialysis is used for ECF sampling for analysis of a single anti-cancer agent. However, the resistant nature of some CNS tumors renders combination regimens a plausible approach to overcome tumor resistance (Brown et al., 2008; Fan and Weiss, 2010; Karpel-Massler et al., 2011; Krakstad and Chekenya, 2010; Prados et al., 2006). To our knowledge, no studies have been published that show the feasibility of simultaneous microdialysis sampling of multiple anti-cancer agents. Thus, a robust method to simultaneously sample multiple anti-cancer agents coupled with sensitive analytical 
methods can prove useful in determining the penetration profile of anti-cancer agents administered in combination.

Microdialysis has many advantages over other methods to assess CNS drug disposition, but it does have limitations. A limitation of microdialysis is the trauma associated with surgical insertion of the probe in the tissue. This raises some concerns to whether inserting the probe may damage the endothelial cells of the BBB and thus limit the suitability of microdialysis to study BBB-related questions. Several studies have evaluated the trauma associated with implanting a microdialysis probe. For example, cerebral blood flow was disturbed 2 hours after probe implantation in rat brains. After 24 hours, cerebral blood flow is restored and no major disturbances could be detected (Benveniste et al., 1987). For cerebral tumors, implanting microdialysis probes showed no signs of inflammation for $\sim 3$ days. After 3 days of implantation, a mild tissue reaction manifested as gliosis was detected around the probe. This reaction was shown not to affect BBB transport (de Lange et al., 1994). Generally, histological evaluation performed in several microdialysis studies showed only mild tissue trauma, if any, after probe implantation, which is not likely to affect BBB integrity (Carcaboso et al., 2010; Elmeliegy et al., 2011a; Elmeliegy et al., 2011b). Finally, if probe implantation damages the BBB, one would speculate that substances that typically cannot penetrate to the brain would show higher brain accumulation after probe implantation. However, brain accumulation of integrity markers such as fluorescein dextran and ${ }^{14} \mathrm{C}$-alpha-aminoisobutyric acid was not increased after probe implantation indicating that BBB integrity was preserved (Benveniste et al., 1984; de Lange et al., 1998).

Several microdialysis studies have been conducted in orthotopic glioma xenografts (Ma et al., 2003; Ma et al., 2001). However, GEMMs have never been used in microdialysis studies. Several reasons may contribute to the difficulty of using GEMMs in microdialysis studies. One reason is the need for complex and costly imaging techniques to determine brain tumor location. Such expensive imaging modalities (e.g. MRI) are not necessarily available in all research institutes.

Another reason is the technical challenge of implanting the microdialysis cannula and probe in the spontaneously developed tumor given the heterogeneity commonly associated with these models. Implanting microdialysis cannulas in orthotopic xenograft models is relatively easy and can be established by stereotactically injecting tumor cells followed by microdialysis cannula/probe using the intersection between the coronal and sagittal sutures, i.e. the bregma point, as a reference point (Carcaboso et al., 2010; Ma et al., 2003). However, the bregma point does not appear on images derived by MRI. The lack of common reference points between the MRI images and the mouse skull renders accurate determination of implantation coordinates in GEMMs rather challenging. New methodologies to guide microdialysis cannula implantation using a reference point visible on MRI may prove useful for CNS penetration studies in GEMMs.

Another limitation that prevented the use of cerebral microdialysis in GEMMs is that, in many cases identification of the tumor can only be accomplished at a late stage at which surgical implantation of the cannula and performing the microdialysis experiment 
is technically not feasible. These problems can be addressed using novel imaging techniques such as MRI to identify the tumor location prior to surgically implanting the cannula.

During a microdialysis experiment, drug concentration in tissue ECF is more than in the perfusate and therefore true equilibrium conditions cannot be achieved. Thus, concentrations in the dialysate will be different from that in tissue ECF. Therefore, relating dialysate concentrations to tissue ECF concentrations, i.e. estimating the extent of drug recovery from tissue ECF through the probe, is an important step in the design of the microdialysis experiment. Several methods to calculate probe recovery have been reported (reviewed in de Lange et al., 1997). We will limit our discussion to two of the major recovery determination methods, namely retrodialysis and zero flow rate.

\subsubsection{Retrodialysis}

In this method, a known concentration of the drug is prepared in the perfusate and pumped through the probe. The basic assumption of the retrodialysis method is that the diffusion process across the microdialysis probe is quantitatively equal in both directions i.e. the percentage of the analyte lost from the perfusate to the medium is equal to the percentage recovered from the medium to the perfusate (Figure 1-5) (Wang et al., 1993b). This method should be conducted when there is no drug present in the body, e.g. before administering the drug.

\subsubsection{Zero flow rate}

The extent of recovery is inversely proportional to the flow rate of the perfusate i.e. the lower the flow rate, the higher the probe recovery (Jacobson and Hamberger, 1985; Johnson and Justice, 1983; Lindefors et al., 1989). The zero flow rate (ZFR) method is applied by collecting dialysates at several flow rates, measuring different dialysate concentrations, and plotting the concentrations against the flow rate. The basic hypothesis of this method is that extrapolation to a hypothetical flow rate of zero provides an estimate of the absolute drug concentration in tissue ECF (Jacobson et al., 1985). The ZFR requires drug concentration to remain relatively constant while collecting the dialysates at different flow rates (Jacobson et al., 1985).

\subsection{SUMMARY}

For the past three decades, little progress has been achieved in the treatment of malignant glioma in both adults and pediatric populations. The recent advances in tumor genotyping identified important genetic alterations in disease development and progression. However, several clinical trials showed that molecularly targeted agents that target these genetic alterations failed to provide significant therapeutic benefit for malignant glioma patients. Inadequate penetration of these agents to the tumor may 

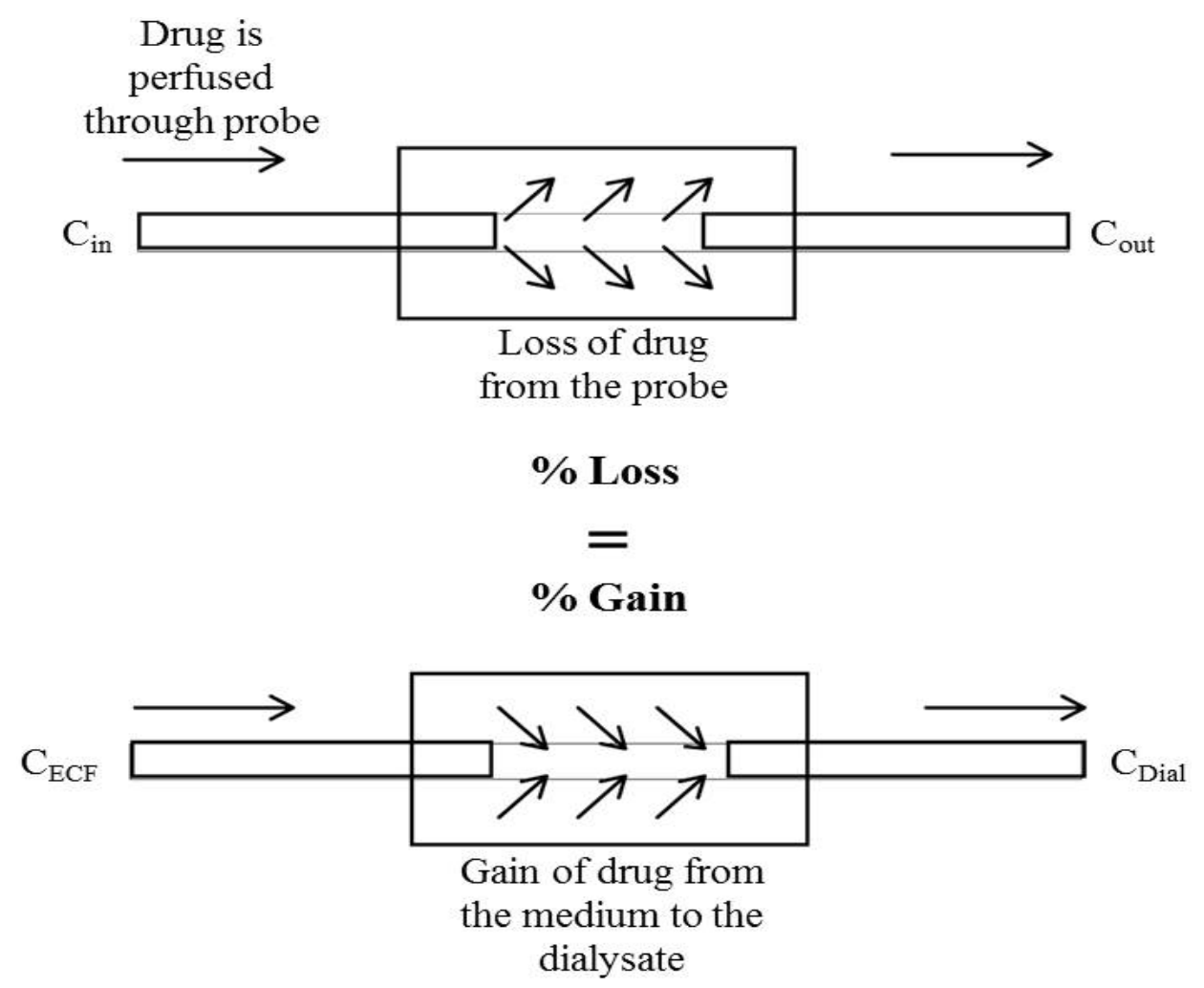

\section{Figure 1-5. Diagrammatic illustration of the retrodialysis method}

Retrodialysis experiment (upper panel) is conducted by perfusing drug prepared in perfusate $\left(\mathrm{C}_{\mathrm{in}}\right)$ through the probe. A fraction of the drug will be lost across the probe. Percent of drug lost through probe can be calculated as $100 \mathrm{X}\left(\frac{\text { Cin-Cout }}{\text { Cin }}\right)$. The underlying assumption of this method is that the percentage of drug lost across the membrane is equal to that gained from the tissue ECF during the experiment (lower panel).

Source: Reprinted by permission from Elsevier. de Lange et al. (2000) Methodological issues in microdialysis sampling for pharmacokinetic studies. Advanced Drug Delivery Reviews 45:125-148. 
partially explain the failure encountered in the clinics. Delivery of these agents to the tumor can be influenced by several efflux and uptake transporters expressed at the BBB and the BCSFB. Through this project, we investigated the effect of major efflux and uptake transporters at the $\mathrm{BBB}$ and the BCSFB on the penetration of two tyrosine kinase inhibitors, erlotinib and crenolanib. We also studied erlotinib and crenolanib penetration in murine models of high-grade glioma using cerebral microdialysis.

\subsection{SPECIFIC AIMS}

The objective of specific aim 1 was to assess the role of efflux and uptake transporters in erlotinib CNS penetration and intracellular accumulation.

The objective of specific aim 2 was to use MRI imaging to identify a reference point for implanting microdialysis cannula in spontaneously arising tumors in a GEMM for high-grade glioma.

The objective of specific aim 3 was to demonstrate the feasibility of simultaneous sampling of multiple anti-cancer agents, erlotinib and crenolanib, via cerebral microdialysis. 


\section{CHAPTER 2. ROLE OF ATP-BINDING CASSETTE AND SOLUTE CARRIER TRANSPORTERS IN ERLOTINIB CNS PENETRATION AND INTRACELLULAR ACCUMULATION*}

\subsection{INTRODUCTION}

Erlotinib (Tarceva ${ }^{\mathrm{TM}}$ ) is an orally administered epidermal growth factor receptor (EGFR) inhibitor approved for certain types of non-small cell lung cancer (NSCLC) and pancreatic cancer (Shepherd et al., 2005). In humans, erlotinib is extensively metabolized primarily by hepatic CYP3A4 and CYP3A5 with some metabolism by CYP1A2 and CYP2C8 (Ling et al., 2006). The metabolite, OSI-420, retains EGFR tyrosine kinase inhibitory activity as well as in vivo tumor growth inhibitory activity (Moyer et al., 1997).

Clinical trials have evaluated the safety and activity of erlotinib in patients with primary and secondary central nervous system (CNS) tumors with frequent EGFR alterations, such as high-grade glioma and brain metastases of NSCLC (Broniscer et al., 2009; Sun et al., 2009). In most of these studies, erlotinib failed to show clinically significant activity (Broniscer et al., 2009; Shepherd et al., 2005; van den Bent et al., 2009a). A potential explanation for this lack of efficacy is that the BBB prevents the accumulation of effective erlotinib concentrations in the tumor (Loscher and Potschka, 2005; Shen et al., 2004). This is supported by evidence from clinical studies showing low erlotinib and OSI-420 accumulation in high-grade glioma (Lassman et al., 2005; Raizer et al., 2010a).

One mechanism by which the BBB limits erlotinib CNS penetration is the expression of efflux proteins of the ATP-binding cassette (ABC) transporter family at the brain endothelial cells (Giacomini et al., 2010). ABC transporters likely to impede erlotinib CNS distribution include P-glycoprotein (P-gp/MDR1/ABCB1; Mdr1a/b in mice), breast cancer resistance protein (BCRP/ABCG2; Bcrp1/Abcg2 in mice), and the multidrug resistance-associated protein 4 (MRP4/ABCC4; Mrp4/Abcc4 in mice) (Kodaira et al., 2010; Leggas et al., 2004a). Expression of efflux transporters on tumor cell membranes would be an additional mechanism of drug resistance preventing the intracellular penetration of anti-cancer drugs (Carcaboso et al., 2010).

In addition to $\mathrm{ABC}$ transporters, uptake transporters located at the $\mathrm{BBB}$ or at the blood-tumor barrier could also regulate drug delivery to the CNS and brain tumors (Ose et al., 2010). Several uptake transporters of the SLC22A family are localized at the BBB or at the blood cerebrospinal fluid barrier (BCSFB) where they affect the disposition of

* Reprinted with permission. Elmeliegy MA et al. (2011) Role of ATP-binding cassette and solute carrier transporters in erlotinib CNS penetration and intracellular accumulation. Clin Cancer Res 17:89-99. 
their substrates (Kikuchi et al., 2003; Kusuhara and Sugiyama, 2005; Lin et al., 2010; Nagata et al., 2002).

The expression of some uptake transporters is detected in vessels of high-grade glioma where they may affect drug accumulation inside the tumor (Bronger et al., 2005). However, the affinity of these transporters towards many anti-cancer agents, including erlotinib, has not been studied.

Erlotinib concentrations in the cerebrospinal fluid (CSF) of patients with brain tumors have been previously reported as $8 \%$ of the total plasma concentration (Broniscer et al., 2007b; Togashi et al.). However, several studies in animals have demonstrated differential (usually increased) drug distribution in the CSF as compared to the brain parenchyma extracellular fluid (ECF) (Kaddoumi et al., 2007; Shen et al., 2009; StainTexier et al., 1999), emphasizing the limitations of using CSF sampling, or whole brain homogenates, to examine CNS drug penetration.

In the present study, we used cerebral microdialysis sampling to assess the role of murine ABC transporters Bcrp1, P-gp, and Mrp4 in CNS penetration of erlotinib and OSI-420. Furthermore, we used a panel of cell lines expressing human efflux or uptake transporters to investigate transport processes involved in CNS penetration and intracellular accumulation of erlotinib and OSI-420.

\subsection{MATERIALS AND METHODS}

\subsubsection{Animals}

Animals used were wild-type FVB, $A b c g 2^{--}, M d r 1 a / b^{--}, A b c c 4^{--}$, and $M d r l a / b^{-/-}$ $A b c g 2^{-/-}$mice. All mice were on a FVB genetic background, 3-4 month old, females, and provided by Taconic (Hudson, NY; except $A b c c 4^{-/}$, obtained at SJCRH). All animal studies were conducted using protocols and conditions approved by the Institutional Animal Care and Use Committee.

\subsubsection{Chemicals and reagents}

GF120918 was purchased from API (China). Erlotinib, OSI-420 analytical standards, and the internal standards for each compound used in the mass spectrometry assay were kindly provided by OSI pharmaceuticals (Uniondale, NY). 2-Hydroxypropyl- $\beta$-cyclodextrin (HPBCD) was purchased from CTD, Inc. (High Springs, FL). 


\subsubsection{Cell lines}

The porcine kidney epithelial LLC-PK1 cell line and the L-MDR1 cell lines stably expressing human ABCB1 were cultured in DMEM, 10\% fetal bovine serum, penicillin $100 \mathrm{U} / \mathrm{mL}$ and streptomycin $100 \mu \mathrm{g} / \mathrm{mL}$; all from Invitrogen (Carlsbad, CA). Saos 2 cells containing pcDNA3.1 empty vector, ABCG2, or ABCC4 were maintained in DMEM containing $10 \%$ fetal bovine serum, penicillin $(100 \mathrm{U} / \mathrm{mL})$, streptomycin $(100$ $\mu \mathrm{g} / \mathrm{mL}$ ), and G418 sulfate (500 $\mu \mathrm{g} / \mathrm{mL}$; Invitrogen). HEK-293 cells stably transfected with OCT1, OCT2 (Hayer-Zillgen et al., 2002), OAT1, OAT2, OAT3 (Tahara et al., 2006), OCTN1, and OCTN2 (Nezu et al., 1999), along with pcDNA vector-transfected controls were cultured in DMEM supplemented with 10\% fetal bovine serum and G418 sulfate $(400-800 \mu \mathrm{g} / \mathrm{mL})$.

\subsubsection{Determination of erlotinib and OSI-420 protein binding in mouse plasma}

Erlotinib and OSI-420 were added to mouse plasma (Hiltop, Scottdale, PA) to make final erlotinib and OSI-420 concentrations of 1,2 , and $4 \mu \mathrm{g} / \mathrm{mL}$. $200 \mathrm{uL}$ of plasma were added to each well of a 96-well equilibrium dialysis plate (Harvard Apparatus, MA) and incubated on a rotator at $37^{\circ} \mathrm{C}$. Samples were collected from plasma and PBS buffer chambers at several time points after starting the experiment. Samples were analyzed using a validated liquid chromatography/mass spectrometry (LC/MS) method (Zhao et al., 2003). Unbound fraction $\left(f_{\mathrm{u}}\right)$ of either erlotinib or OSI-420 was calculated as follows:

$$
f_{\mathrm{u}}=\frac{\mathrm{C}_{\mathrm{PBS}}}{\mathrm{C}_{\text {Plasma }}}
$$

where $\mathrm{C}_{\mathrm{PBS}}$ and $\mathrm{C}_{\mathrm{Plasma}}$ are the concentrations of erlotinib or OSI-420 in PBS and plasma, respectively. To compare the unbound fraction of erlotinib and OSI-420 in different strains, plasma was isolated from each strain and equilibrium dialysis was performed as previously described.

\subsubsection{Erlotinib and OSI-420 plasma pharmacokinetics}

A single erlotinib dose was administered to all five strains of mice $(\mathrm{n}=8-13$ mice per group). To administer erlotinib, Tarceva ${ }^{\mathrm{TM}}$ (OSI Pharmaceuticals, Uniondale, NY) tablets were pulverized and suspended in $0.2 \%$ carboxymethylcellulose and $0.05 \%$ Tween 20, for a final concentration of $5 \mathrm{mg} / \mathrm{mL}$. The erlotinib suspension was administered at a dosage of $50 \mathrm{mg} / \mathrm{kg}$ by oral gavage. Plasma samples were taken by retro-orbital bleeding at 7 time points after drug administration ( 5 minutes, $0.5 \mathrm{hrs}, 1,2$, 6,12 , and 24 hours). At least four different animals contributed to each time point. Blood samples were centrifuged at $3000 \mathrm{rpm}$ for 3 minutes. Plasma samples were immediately stored at $-80^{\circ} \mathrm{C}$ till further analysis using liquid chromatography/tandem mass spectrometry (LC MS/MS) (Zhao et al., 2003). 


\subsubsection{Development of pharmacokinetic limited sampling models for erlotinib and OSI-420}

Data from the plasma pharmacokinetic experiment were analyzed with nonlinear mixed-effects modeling using the importance sampling EM algorithm in NONMEM VII (Beal and Sheiner, 1980). A model with one compartment for erlotinib and one compartment for OSI-420 and first-order conversion from erlotinib to OSI-420 and firstorder elimination of OSI-420 was used to fit the plasma concentration-time data. Models with first-order absorption, zero-order absorption and sequential zero-order and firstorder absorption were tested. The appropriate model was chosen based on objective function value (OFV) and inspection of goodness-of-fit plots. The estimated parameters included the duration of zero-order infusion (D1), first-order absorption rate constant $\left(\mathrm{k}_{\mathrm{a}}\right)$ apparent oral clearance of erlotinib $\left(\mathrm{CL}_{\mathrm{ERL}} / \mathrm{F}\right)$, apparent volume of distribution of erlotinib $\left(\mathrm{V}_{\mathrm{ERL}} / \mathrm{F}\right)$, apparent clearance of OSI-420 $\left(\mathrm{CL}_{\mathrm{OSI}} / \mathrm{FE}\right)$, and apparent volume of distribution of OSI-420 ( $\left.\mathrm{V}_{\mathrm{OSI}} / \mathrm{FE}\right)$, where $\mathrm{F}$ is the bioavailability and $\mathrm{FE}$ is the fraction of erlotinib converted to OSI-420. Inter-individual variability (IIV) terms were added to the $\mathrm{CL}_{\mathrm{ERL}} / \mathrm{F}, \mathrm{V}_{\mathrm{ERL}} / \mathrm{F}, \mathrm{CL}_{\mathrm{OSI}} / \mathrm{FE}$, and D1 parameters. IIV was modeled as a log-normal distribution and a proportional error model was used for residual variability.

Data below the lower limit of quantitation of $1 \mathrm{ng} / \mathrm{mL}$ were included in the analysis with a likelihood-based approach using method M3 as previously described (Ahn et al., 2008). Each strain of mice was analyzed separately, and the model parameters were used to develop a limited sampling strategy using the D-optimality algorithm in ADAPT 5 (D'argenio et al., 2009). The limited sampling strategy consisted of three time points at which plasma samples could be drawn in each microdialysis experiment in order to estimate the plasma exposure using the population priors.

\subsubsection{In vitro probe recovery studies}

To study our ability to dialyze erlotinib and OSI-420 as well as strategies required to enhance their relative recovery through microdialysis probes, a stock solution containing $1 \mu \mathrm{g} / \mathrm{mL}$ erlotinib and $0.5 \mu \mathrm{g} / \mathrm{mL}$ OSI-420 in artificial CSF (aCSF, Leggas et al., 2004b) was prepared. A $1 \mathrm{~mm}$ microdialysis probe (MBR-1-5 brain probe, BASi, IN) was inserted into this solution and perfused with: (a) aCSF, (b) 10\% HPBCD in aCSF, and (c) bovine serum albumin (BSA; 4\% in aCSF) for 1 hour followed by $10 \%$ HPBCD in aCSF. To assess the effect of flow rate on the recovery of erlotinib and OSI-420 from the stock solution, perfusates were pumped through the probe at $0.3,0.5,1$, and $2 \mu \mathrm{L} / \mathrm{min}$ and dialysates were collected. Dialysates and stock solution samples were analyzed using LC MS/MS. Relative recovery (\%) was calculated as $C_{\text {Dial }} / C_{\text {stock }} * 100$, where $C_{\text {Dial }}$ is the erlotinib or OSI-420 concentration in the dialysates and $\mathrm{C}_{\text {stock }}$ is the erlotinib or OSI-420 concentration in the stocks. All in vitro recovery experiments were performed at room temperature under stirred conditions. 


\subsubsection{Erlotinib brain microdialysis studies}

In vivo microdialysis experiments were conducted to sample erlotinib and OSI-420 in the ECF of the mouse brain after a single $50 \mathrm{mg} / \mathrm{kg}$ oral dose of erlotinib suspended in $0.2 \%$ carboxymethylcellulose and $0.05 \%$ Tween 20 . First, a microdialysis guide cannula (MD-2255, Bioanalytical Systems- West Lafayette, IN) was inserted vertically into the striatum in the cerebral cortex $(1.1 \mathrm{~mm}$ anterior to bregma suture, 1.1 $\mathrm{mm}$ lateral to sagittal suture, $2.0 \mathrm{~mm}$ ventral). The surgery was performed under anesthesia (100 mg/kg ketamine and $10 \mathrm{mg} / \mathrm{kg}$ xylazine) as previously reported (Shen et al., 2009; Zhuang et al., 2006). Mice recovered for at least 3 days before the microdialysis experiment. The day of the experiment, a 1-mm-length microdialysis probe (MBR-1-5, BASi, IN) was primed and flushed with aCSF containing 10\% HPBCD. The newly primed probe was then slowly inserted in the guide cannula and allowed to equilibrate for 1 hour at $0.5 \mu \mathrm{L} / \mathrm{min}$. Under slight isoflurane anesthesia ( $2.5 \%$ in oxygen), the mouse received a $50 \mathrm{mg} / \mathrm{kg}$ dose of erlotinib, by oral gavage. Then, dialysates were collected for 24 hours, using a fraction collector CMA 142 (North Chelmsford, MA).

We used the limited sampling strategy described above to collect plasma samples. At the end of the experiment, the animal was euthanized and the brain was fixed in $10 \%$ neutral buffered formalin for 72 hours and embedded in paraffin. H\&E-stained sections $(4 \mu \mathrm{m})$ were examined microscopically to examine the brain tissue surrounding the cannula and probe.

\subsubsection{In vivo microdialysis probe recovery using zero flow rate (ZFR) method}

After the first 5 hours of sample collection, dialysates were collected at different flow rates $0.2,0.5,1$, and $4 \mu \mathrm{L} / \mathrm{min}$. Dialysate concentrations were allowed to stabilize for 10-15 min between each change. The recovery experiment lasted 2.5 hours after which the flow rate was set back to $0.5 \mu \mathrm{L} / \mathrm{min}$ for the remainder of the experiment. Samples were analyzed using LC MS/MS and concentrations were plotted against flow rate. Extrapolation to zero flow rate was performed as previously described (Menacherry et al., 1992) using non-linear regression analysis in GraphPad Prism version 5.0b (GraphPad Software, San Diego, CA) using the following formula:

$$
\mathrm{C}_{\text {Dial }}=\mathrm{C}_{0} * \exp (-\mathrm{rA} * \mathrm{X})
$$

where $\mathrm{r}$ (mass transport coefficient) and A (surface area of the dialysis membrane) are constants, estimated using non-linear regression, $\mathrm{C}_{0}$ represents erlotinib or OSI-420 concentrations in the dialyzed tissue, $\mathrm{C}_{\text {Dial }}$ is erlotinib or OSI-420 concentration in dialysates, and $\mathrm{X}$ is the flow rate at which the dialysate was obtained at different flow rates. Since the microdialysis experiment was performed at $0.5 \mu \mathrm{L} / \mathrm{min}$ perfusion rate, in vivo recovery was calculated as: $\mathrm{C}_{\text {Dial at } 0.5 \mu \mathrm{L} / \mathrm{min}} / \mathrm{C}_{0}$ (Menacherry et al., 1992). 


\subsubsection{Pharmacokinetic analysis of microdialysis studies}

A pharmacokinetic model was fit simultaneously to the plasma and brain ECF microdialysis data. In order to incorporate information from the previous plasma pharmacokinetic experiment, pharmacokinetic data from both experiments was pooled and analyzed together. Modeling was performed with NONMEM VII using the importance sampling EM algorithm. The model was based on the model described above for plasma data, with additional compartments for erlotinib and OSI-420 in brain ECF. In addition to the previous parameters, this model included first-order rate constants describing erlotinib transfer into and out of the brain ECF (k24 and k42), first-order rate constants describing the transfer of OSI-420 into and out of the brain ECF (k35 and k53), and a single volume of distribution parameter for erlotinib and OSI-420 in the brain ECF (Vbrain). IIV was included on D1, CLERL/F, VERL/F, CLOSI/FE, VOSI/FE, and intercompartment rate parameters.

Individual post-hoc parameters were used to simulate the concentration-time curve from 0 to $24 \mathrm{~h}$ in plasma and brain ECF for erlotinib and OSI-420 for each mouse from which the area under the concentration-time curves for plasma and brain $\left(\mathrm{AUC}_{0-24, \text { Plasma }}\right.$ and $\left.\mathrm{AUC}_{0-24, \mathrm{Brain}}\right)$ were calculated with the log-linear trapezoidal method. Unbound plasma $\mathrm{AUC}$ ( $\mathrm{AUC}_{0-24, \mathrm{uPlasma}}$ ) for erlotinib and OSI-420 were obtained by multiplying $\mathrm{AUC}_{0-24 \text {,Plasma }}$ by the appropriate unbound fraction $(\mathrm{fu})$. The extent of brain penetration $\left(P_{\text {Brain }}\right)$ for erlotinib and OSI-420 was calculated as the brain ECF-to-unbound plasma AUC ratio:

$$
P_{\text {Brain }}=\frac{\mathrm{AUC}_{0-24, \text { Brain }}}{\mathrm{AUC}_{0-24, \mathrm{uPlasma}}}
$$

Differences in $P_{\text {Brain }}$ between wild-type mice and other mouse strains were assessed using the Mann-Whitney test.

\subsubsection{Intracellular drug accumulation studies}

To study the role of several efflux and influx transporters in erlotinib intracellular accumulation, we used an array of cell lines expressing specific efflux or influx transporters and compared the accumulation of erlotinib or OSI-420 in the cell lines overexpressing each transporter to that transfected with an empty vector. Intracellular accumulation of erlotinib and OSI-420 were measured in Saos2 cells transfected with human BCRP or MRP4 and LLC-PK1 cells transfected with MDR1, in presence or absence of the P-gp/BCRP inhibitor, elacridar.

Uptake experiments were done using HEK293 cells transfected with cDNAs coding for members of human OCT, OAT, and OCTN families. Briefly, 5 x $10^{5}$ cells of each cell line were plated in 6-well plates in triplicates. Cells were allowed to attach overnight. For cells expressing the OAT1, 2, 3, and their vector control, sodium butyrate $(5 \mu \mathrm{mol} / \mathrm{L})$ was added to the medium for $24 \mathrm{~h}$ to induce expression of the respective 
transporter genes (Tahara et al., 2006). At the day of experiment, medium was removed and cells were incubated with media containing $0.5 \mu \mathrm{g} / \mathrm{mL}$ of either erlotinib or OSI-420 at $37^{\circ} \mathrm{C}$. At a predetermined time interval, the experiment was terminated by removing the incubation medium and adding ice-cold PBS. Cells were washed twice with ice-cold PBS, gently scraped, collected, and centrifuged for 4 minutes at $3000 \mathrm{rpm}$ at $0^{\circ} \mathrm{C}$. Subsequently, cells were resuspended in $100 \mu \mathrm{L}$ of $5 \mathrm{mM}$ ammonium formate for cell lysis. The BCA assay was used to determine protein concentrations. Erlotinib and OSI-420 concentrations in the lysis supernatants were determined using LC MS/MS (Zhao et al., 2003).

\subsection{RESULTS}

\subsubsection{Erlotinib and OSI-420 protein binding in mouse plasma}

Using equilibrium dialysis, we assessed the extent of plasma protein binding for erlotinib and OSI-420. The range of concentrations used was similar to those expected after administering erlotinib orally to mice at $20 \mathrm{mg} / \mathrm{kg}$. The time after which the dialysis reached equilibrium was 10 hours. Erlotinib, as well as its metabolite, was found to be highly protein bound in mouse plasma. Unbound fraction $\left(f_{\mathrm{u}}\right)$ of erlotinib was $4.8 \% \pm$ $0.7 \%$ and for OSI-420 was $6.6 \% \pm 0.7 \%$. No significant differences were found in unbound fractions of erlotinib or OSI-420 between strains (Table 2-1).

\subsubsection{Limited sampling models for erlotinib and OSI-420}

The erlotinib dosage used in preclinical studies has ranged from 5 to 150 $\mathrm{mg} / \mathrm{kg} /$ day orally for up to 4 weeks (Marchetti et al., 2008; Sarkaria et al., 2007). In our studies, we sought to use an erlotinib dosage that achieves a systemic exposure similar to that observed in patients (Broniscer et al., 2009; Broniscer et al., 2007b). First, we conducted a plasma pharmacokinetic study of a single erlotinib dose administered orally at a dosage of $50 \mathrm{mg} / \mathrm{kg}$ in wild-type, $\mathrm{Abcg} 2^{-/-}, \mathrm{Mdrla} / \mathrm{b}^{-/-}, \mathrm{Mdrla} / \mathrm{b}^{-/-} \mathrm{Abcg} 2^{-/-}$, and $\mathrm{Abcc4^{- }}$ ${ }^{/-}$mice. This single dose of erlotinib was tolerated well by all strains.

Between the different absorption models, a sequential zero-order and first-order model described the data best based on goodness-of-fit plots and lowered the OFV between 6 and 35 units for different strains compared to first-order absorption alone and lowered the OFV between 9 and 33 units for different strains for zero-order absorption alone. The final model is shown in Figure 2-1. Concentration-time plots for erlotinib, OSI-420, along with the model-predicted concentrations are shown in Figure 2-2 and Figure 2-3. Pharmacokinetic parameters for all strains are listed in Table 2-2.

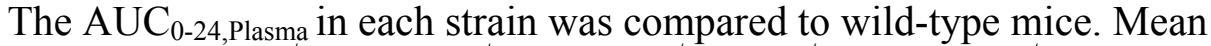
$\mathrm{AUC}_{0-24, \mathrm{Plasma}}$ for $\mathrm{Abcg} 2^{-/-}, \mathrm{Mdrla} / \mathrm{b}^{-/-}, \mathrm{Mdrla} / \mathrm{b}^{-/-} \mathrm{Abcg} 2^{-/-}$, and $\mathrm{Abcc} 4^{-/-}$strains were 1.25, $0.9,1.3,0.9$ fold compared to that of wild-type mice. Using the population parameters, 
Table 2-1. Unbound fractions $(f u)$ in plasma of different mouse strains

\begin{tabular}{lll}
\hline Strain & Erlotinib $\left(\boldsymbol{f}_{\mathbf{u}}\right)$ & OSI-420 $\left(\boldsymbol{f}_{\mathbf{u}}\right)$ \\
\hline Wild-type & $5.24 \pm 0.8$ & $7.47 \pm 1.75$ \\
Abcg2 & $4 . /-$ & $8.08 \pm 0.13$ \\
Mdrla $/ b^{-/-}$ & $5.59 \pm 1.7$ & $8.46 \pm 0.91$ \\
Mdrla/b/- Abcg2 ${ }^{-/-}$ & $4.47 \pm 0.09$ & $7.33 \pm 0.40$ \\
Abcc4 & $4.58 \pm 0.49$ & $7.12 \pm 0.41$ \\
\hline
\end{tabular}




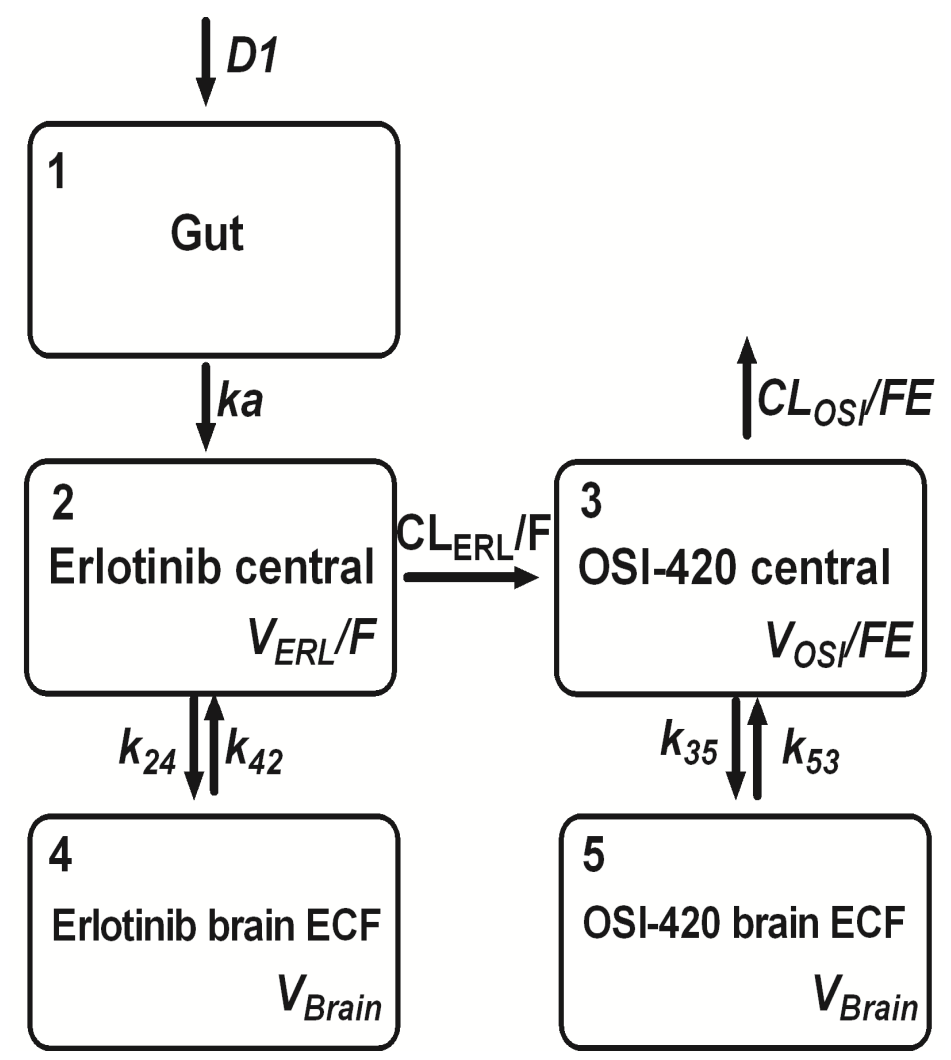

Figure 2-1. Compartmental pharmacokinetic model for erlotinib and OSI-420

D1, duration of zero-order absorption; ka, first-order absorption rate constant; $V_{\text {ERL }} / F$, apparent volume of distribution of erlotinib; $\mathrm{CL}_{\mathrm{ERL}} / \mathrm{F}$, apparent clearance of erlotinib; $\mathrm{V}_{\mathrm{OSI}} / \mathrm{FE}$, apparent volume of distribution of OSI-420; $\mathrm{CL}_{\mathrm{OSI}} / \mathrm{FE}$, apparent clearance of OSI-420; $\mathrm{k}_{24} / \mathrm{k}_{42}$, first-order rate constants of erlotinib in the brain extracellular fluid; $\mathrm{k}_{35} / \mathrm{k}_{53}$, first-order rate constants of OSI-420 in the brain extracellular fluid; $\mathrm{V}_{\text {Brain, }}$ apparent volume of distribution of the brain extracellular fluid. 

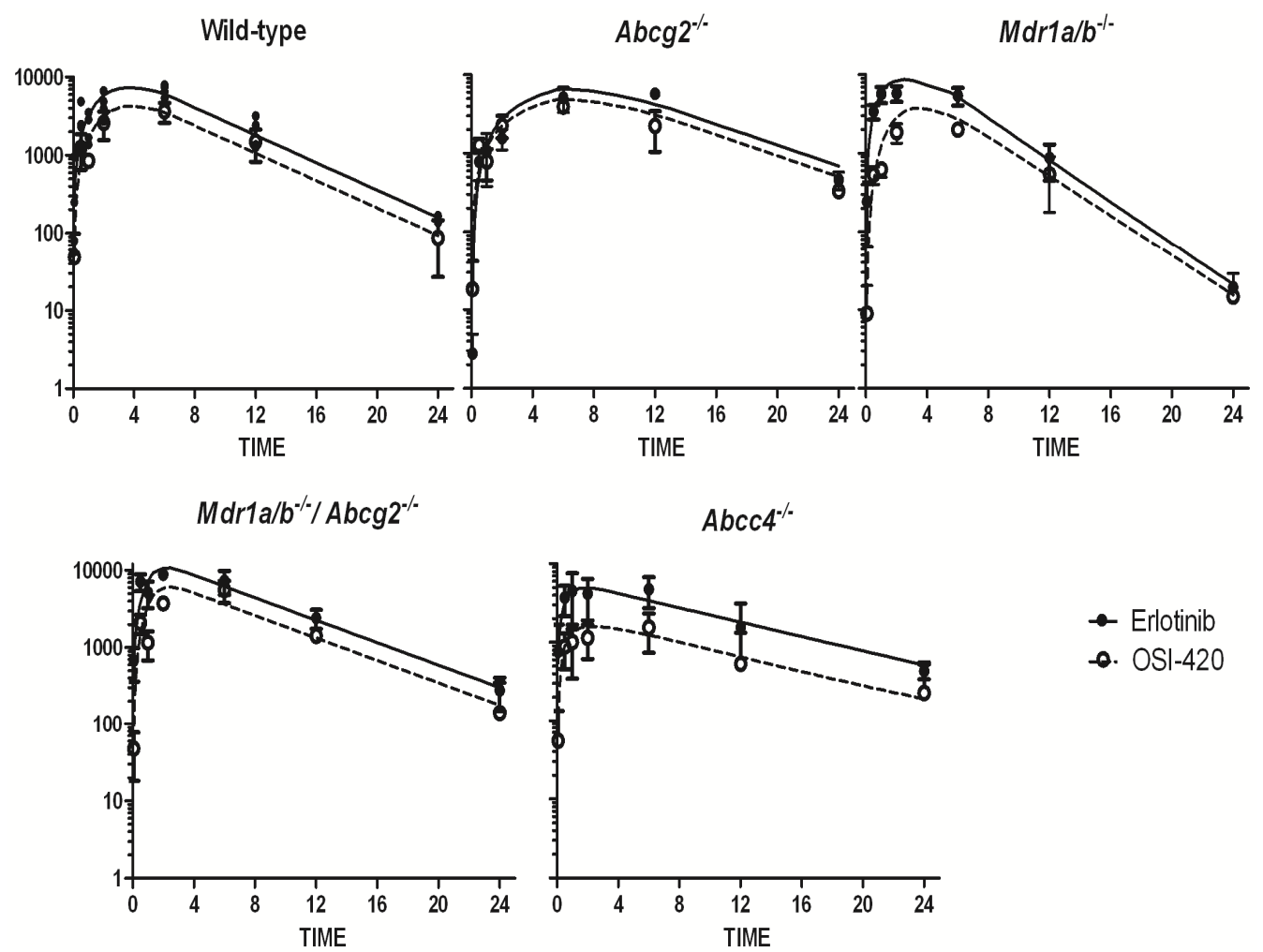

Figure 2-2. Plasma concentration-time plots of erlotinib and OSI-420 after oral administration of a single dose of $50 \mathrm{mg} / \mathrm{kg}$ erlotinib

Model-fitted curves are represented for erlotinib (solid line) and OSI-420 (dashed line). 

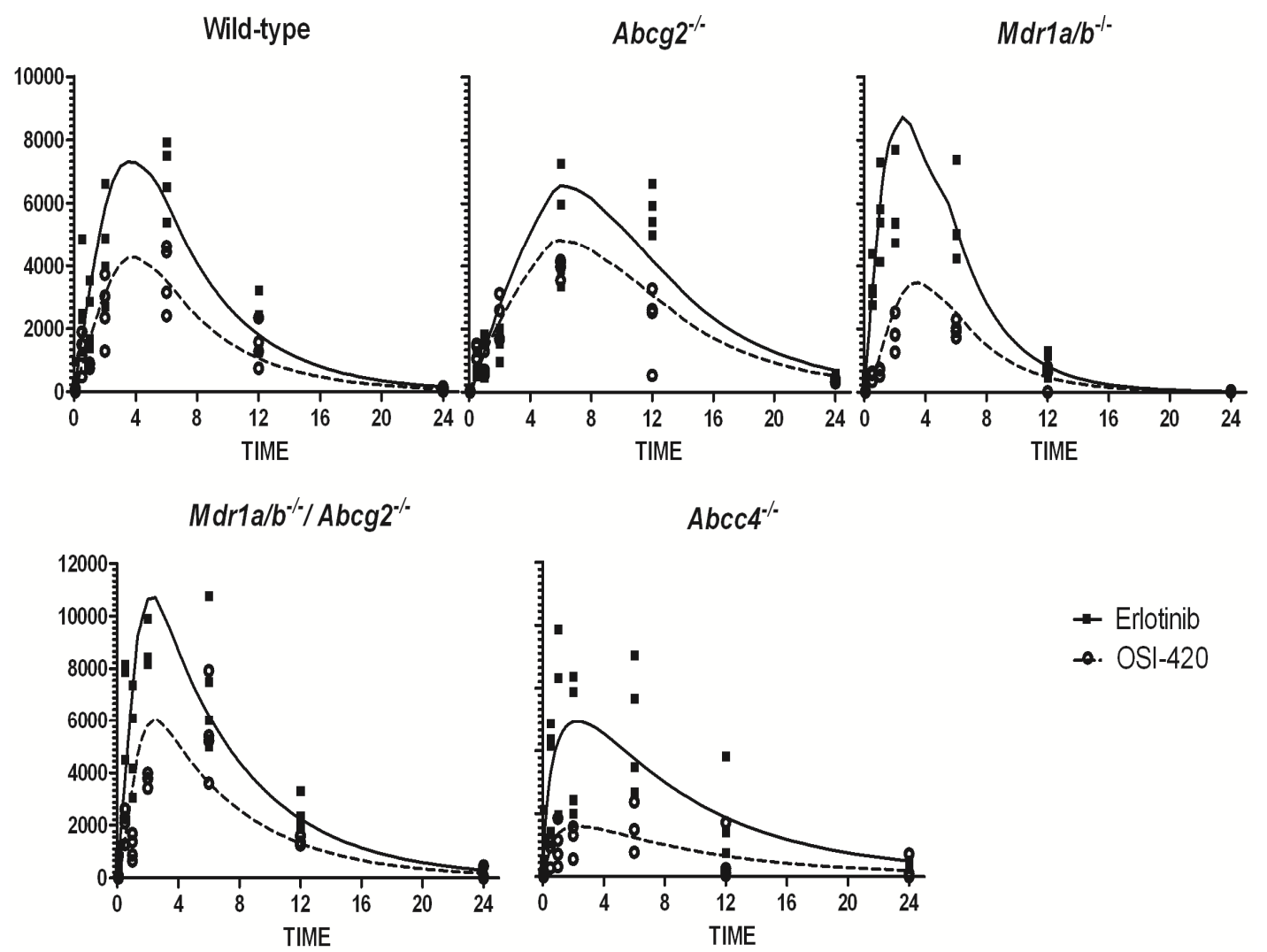

$\rightarrow$ Erlotinib

๑. OSI-420

Figure 2-3. Plasma concentration-time plots on linear scale of erlotinib and OSI-420 after oral administration of a single dose of $50 \mathrm{mg} / \mathrm{kg}$ erlotinib

Model-fitted curves are represented for erlotinib (solid line) and OSI-420 (dashed line). 
Table 2-2. Pharmacokinetic parameters for different mice strains

\begin{tabular}{|c|c|c|c|c|c|}
\hline PK parameters & WT & $\operatorname{Abcg} 2^{-/-}$ & $\operatorname{Mdr} 1 a / b^{-/-}$ & $\operatorname{Mdr1a} / b^{-/-} \operatorname{Abcg} 2^{-/-}$ & $\operatorname{Abcc4^{-/-}}$ \\
\hline \multicolumn{6}{|l|}{$\begin{array}{l}\text { Model Parameters } \\
\text { (Unit) }\end{array}$} \\
\hline D1 $\left(\mathrm{hr}^{-1}\right)$ & 2.74 & 0.6 & 0.82 & 2.33 & 0.806 \\
\hline $\mathrm{Ka}\left(\mathrm{hr}^{-1}\right)$ & 0.21 & 0.049 & 0.19 & 0.12 & 0.202 \\
\hline $\mathrm{CL}_{\mathrm{ERL}} / \mathrm{F}\left(\mathrm{L} \cdot \mathrm{hr}^{-1} \cdot \mathrm{Kg}^{-1}\right)$ & 0.56 & 0.51 & 1.1 & 0.75 & 0.67 \\
\hline $\mathrm{V}_{\mathrm{ERL}} / \mathrm{F}\left(\mathrm{L} . \mathrm{Kg}^{-1}\right)$ & 0.006 & 0.0045 & 0.0009 & 0.021 & 0.0167 \\
\hline $\mathrm{CL}_{\mathrm{OSI}} / \mathrm{FE}\left(\mathrm{L} \cdot \mathrm{hr}^{-1} \cdot \mathrm{Kg}^{-1}\right)$ & 1.07 & 0.95 & 1.5 & 0.549 & 2.14 \\
\hline $\mathrm{V}_{\mathrm{OSI}} / \mathrm{FE}\left(\mathrm{L} . \mathrm{Kg}^{-1}\right)$ & 0.02 & 0.125 & 0.8 & 0.056 & 0.001 \\
\hline $\mathrm{k} 24\left(\mathrm{hr}^{-1}\right)$ & 10 & 0.037 & 8.22 & 0.694 & 9.84 \\
\hline $\mathrm{K} 42\left(\mathrm{hr}^{-1}\right)$ & 5.03 & 3.7 & 4.18 & 1.92 & 2.7 \\
\hline $\mathrm{K} 35\left(\mathrm{hr}^{-1}\right)$ & 0.079 & 0.082 & 0.11 & 0.004 & 0.1 \\
\hline $\mathrm{K} 53\left(\mathrm{hr}^{-1}\right)$ & 0.359 & 0.304 & 0.57 & 0.1 & 0.15 \\
\hline \multicolumn{6}{|c|}{ Inter-individual variability $(\% \mathrm{CV})$} \\
\hline D1 & 67.5 & 2.9 & 71.0 & 36.1 & 48.8 \\
\hline $\mathrm{CL}_{\mathrm{ERL}} / \mathrm{F}$ & 43.4 & 2.5 & 56.8 & 9.6 & 89.5 \\
\hline $\mathrm{V}_{\mathrm{ERL}} / \mathrm{F}$ & 36 & 43.2 & 48.2 & 72 & 44.6 \\
\hline $\mathrm{CL}_{\mathrm{OSI}} / \mathrm{FE}$ & 45.6 & 34.3 & 50.5 & 5 & 37.1 \\
\hline $\mathrm{V}_{\mathrm{OSI}} / \mathrm{FE}$ & 21.5 & 70.3 & 77.4 & 60.7 & 40.1 \\
\hline $\mathrm{k} 24$ & 27.6 & 27.5 & 29.8 & 28.2 & 91.9 \\
\hline $\mathrm{K} 42$ & 31.2 & 11.2 & 44.7 & 42.3 & 66.7 \\
\hline K35 & 16.7 & 21.8 & 19.6 & 50.7 & 71.2 \\
\hline K53 & 26.9 & 31.9 & 3.6 & 33.7 & 46.7 \\
\hline
\end{tabular}


Table 2-2. (continued)

\begin{tabular}{lccccc}
\hline \multicolumn{1}{c}{ PK parameters } & WT & $\boldsymbol{A b c g 2}^{-/-}$ & $\boldsymbol{M d r 1 a}^{-/-}$ & $\boldsymbol{M d r 1 a / b}^{-/-} \boldsymbol{A b c g 2}^{-/-}$ & $\boldsymbol{A b c c 4}^{-/-}$ \\
\hline $\begin{array}{l}\text { Residual Error (\%CV) } \\
\text { Erlotinib }\end{array}$ & 64.8 & 59.8 & 63.2 & 68 & 54.9 \\
OSI-420 & 65.5 & 60.6 & 62.7 & 66.8 & 53.5 \\
\hline
\end{tabular}


we determined the optimal sampling time points to calculate the plasma AUC of erlotinib and OSI-420, constraining to three time points over $18 \mathrm{~h}$. The optimal time points were similar in all strains: one early time point after the administration ( $5 \mathrm{~min})$, one coinciding with the plateau (range from 7.8 to 9 hours), and one late time point (18 hours). For the ease of conducting the experiments, we chose to obtain plasma samples at $0.08,8$, and 18 hours from all strains.

\subsubsection{In vitro microdialysis studies}

Because reproducible dialysis of lipophilic drugs can be difficult using conventional microdialysis methods (Loos et al., 2007; Wang et al., 2008), we anticipated that the microdialysis of erlotinib would require the inclusion of an affinity-based trapping agent, such as HPBCD, in the perfusate. Such modifications in the perfusate have been used before to increase the recovery of poorly dialyzable drugs (Duo et al., 2006). Results of our preliminary in vitro microdialysis experiments supported that erlotinib and OSI-420 had relatively low relative recovery ( 1 and $2 \%$, respectively) using aCSF as a perfusate. The addition of $10 \%$ HPBCD in aCSF improved the recovery of erlotinib and OSI-420 by $\sim 8$-fold (Figure 2-4).

Second, we tested the effect of different flow rates $(0.3,0.5,1$, and $2 \mu \mathrm{L} / \mathrm{min})$ on relative recovery. Increasing the flow rate yielded lower relative recovery. Lastly, we evaluated the effect of perfusing the microdialysis tubes with 4\% BSA to prevent the binding of the drug to the tubes and thus improve the recovery. This strategy did not improve the relative recovery and added complexity due to tube blockage and flow resistance. Thus, we decided to use $10 \% \mathrm{HPBCD}$ in aCSF at $0.5 \mu \mathrm{L} / \mathrm{min}$ at room temperature for the in vivo microdialysis experiments to obtain sufficient recovery with adequate sample volume $(30 \mu \mathrm{L} / \mathrm{h})$. The in vitro recovery at that flow rate was $18 \% \pm$ $2.5 \%$ and $20 \% \pm 1.3 \%$ for erlotinib and OSI- 420 , respectively.

\subsubsection{Zero Flow Rate (ZFR) for estimation of in vivo recovery}

To calculate erlotinib and OSI-420 concentrations in brain ECF, microdialysate concentrations were corrected for probe recovery in each mouse using the ZFR method. This method is based on the fact that at slow perfusion rates and steady state tissue concentrations of the analyte, extrapolation to a hypothetical flow rate value of zero will provide the absolute value of analyte in the dialyzed tissue $\left(\mathrm{C}_{\mathrm{ECF}}\right)$ (Jacobson et al., 1985). Probe recovery can then be calculated by dividing the concentration obtained at the flow rate at which the experiment is conducted (i.e., $\mathrm{C}_{\text {Dial }}$ at $0.5 \mu \mathrm{L} / \mathrm{min}$ ) by $\mathrm{C}_{\mathrm{ECF}}$. First, we validated in vitro the use of the ZFR recovery method for recovery estimation. Dialysates were collected from a stock containing $1 \mu \mathrm{g} / \mathrm{mL}$ of each erlotinib and $0.5 \mu \mathrm{g} / \mathrm{mL}$ OSI-420 at different flow rates and the concentrations were plotted against flow rate. The estimated value of $\mathrm{C}_{\mathrm{ECF}}$ in vitro obtained from extrapolation to zero flow rate using nonlinear regression was $96 \% \pm 15 \%$ and $93 \% \pm 12 \%$ for erlotinib and OSI-420, respectively (results from 6 in vitro experiments; Figure 2-4). The ZFR requires that the concentration 

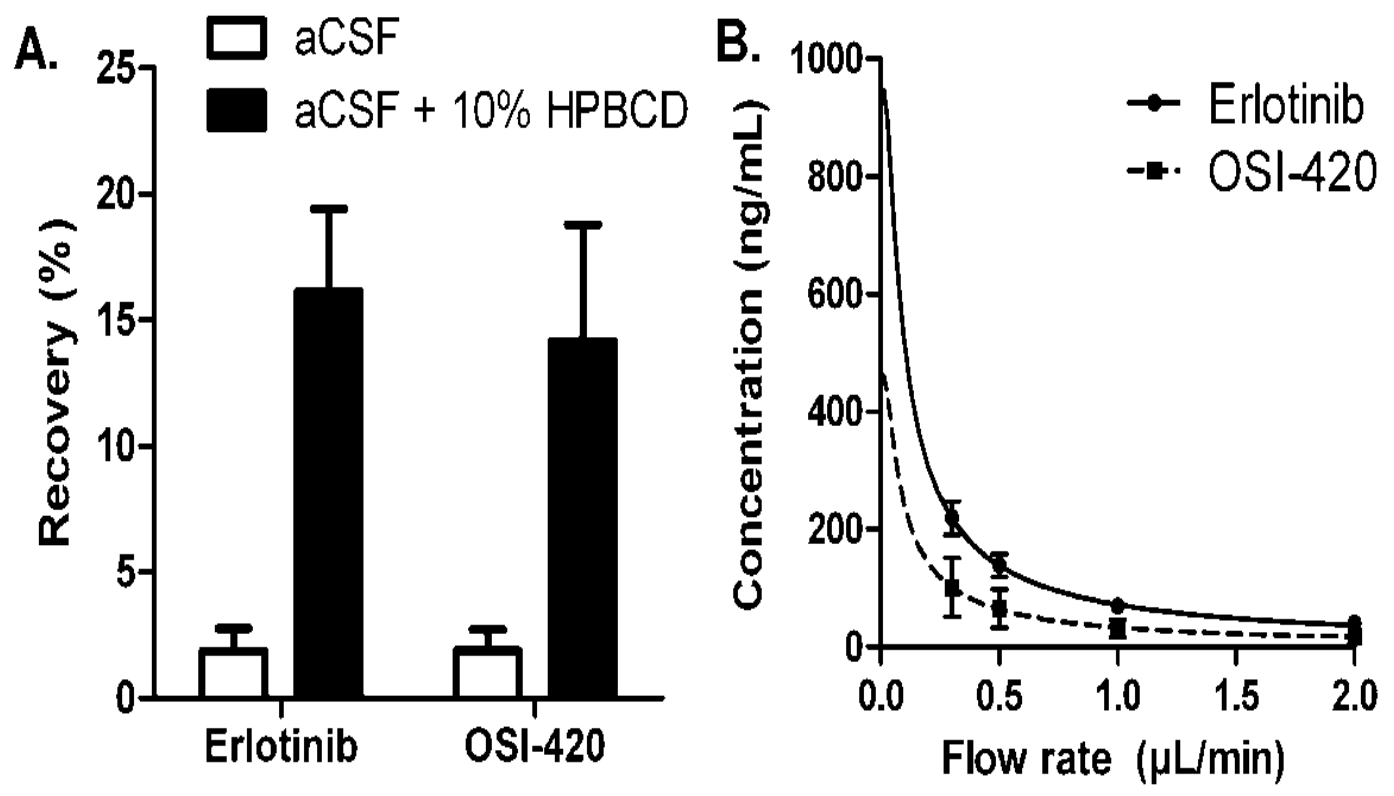

Figure 2-4. In vitro microdialysis studies

A. Including 10\% HPBCD in the perfusate increases recovery for erlotinib and OSI-420 as compared to aCSF alone.

B. In vitro validation of the zero flow rate method. Erlotinib and OSI-420 stocks (1000 and $500 \mathrm{ng} / \mathrm{mL}$, respectively) were prepared; microdialysis probes were inserted in stocks and perfused with 10\% HPBCD in aCSF. Dialysates were collected at different flow rates and concentrations were plotted against flow rate. $\mathrm{C}_{\mathrm{ECF}}$ in vitro was estimated using nonlinear regression to a hypothetical zero flow rate. 
remain relatively constant while collecting the dialysates at different flow rates. Initial microdialysis experiments $(n=4)$ conducted under a stable flow rate of $0.5 \mu \mathrm{L} / \mathrm{min}$ showed that brain ECF concentrations dropped by only $10-15 \%$ over the 5 to $8 \mathrm{hr}$ period. Assuming relatively stable target tissue concentrations, we decided to perform the recovery experiment after 5 hours from drug administration. After the recovery experiment, the flow rate was switched back to $0.5 \mu \mathrm{L} / \mathrm{min}$ and dialysates were collected up to 24 hours. The in vivo recovery for erlotinib was 39\% $\pm 13 \%$ and for OSI-420 was $24 \% \pm 13 \%$.

\subsubsection{Increased ECF penetration of erlotinib and OSI-420 in $A b c g 2^{-/-}$and $M d r 1 a / b^{-}$ ${ }^{1-} \operatorname{Abcg} 2^{-/-}$mice}

Using microdialysis, we sampled brain ECF from wild-type, $\mathrm{Abcg} 2^{-/-}, \mathrm{Mdrla} / \mathrm{b}^{-/}$, Mdrla $/ b^{-/} A b c g 2^{-/-}$, and $A b c c 4^{-/-}$mouse strains after a single oral dose of erlotinib. Brain penetration ratios are shown in Figure 2-5. In wild-type mice, $P_{\text {Brain }}($ mean $\pm \mathrm{SD}$ ) for erlotinib and OSI-420 was $0.27 \pm 0.11$ and $0.07 \pm 0.02$, respectively. $\mathrm{Abcg} 2^{-/-}$mice showed 5-fold higher erlotinib penetration $(1.4 \pm 0.9, p<0.05, \mathrm{n}=5)$ and 7-fold higher OSI-420 penetration $(0.51 \pm 0.04, p<0.05)$ than wild-type mice. However, neither erlotinib penetration $(0.49 \pm 0.3)$ nor OSI-420 penetration $(0.04 \pm 0.02)$ was enhanced in $M d r l a / b^{-/-}$mice ( $\mathrm{n}=4, p>0.05$ for both). In the $M d r l a / b^{-/-} A b c g 2^{-/-}$mice, erlotinib brain penetration was $0.7 \pm 0.26, \sim 3$-fold higher than in wild-type mice $(p<0.01, \mathrm{n}=5)$, and OSI-420 brain penetration $(0.34 \pm 0.21)$ was 4 -fold higher $(p<0.01)$. Brain penetration of $A b c c 4^{-/-}$mice was similar to wild-type mice for both erlotinib $(0.32 \pm 0.24, p>0.05$, $\mathrm{n}=4)$ and OSI-420 $(0.08 \pm 0.06, p>0.05)$. Histological examination verified the localization of the probe track in the brain ECF and indicated no bleeding due to probe insertion (Figure 2-6).

\subsubsection{Intracellular accumulation experiments}

Intracellular accumulation of erlotinib and OSI-420 was significantly reduced in Saos2-BCRP cells as compared to Saos2-pcDNA3.1, indicating the significant role of BCRP in erlotinib transport. Accumulation of OSI-420, but not erlotinib, in LLCPK1-MDR1 cells was significantly reduced as compared to controls (LLCPK1 cells). Saos2-MRP4 cells did not show a significant difference in either erlotinib or OSI-420 accumulation (Figure 2-7). Pre-incubation for 30 minutes with the specific P-gp and BCRP inhibitor, elacridar significantly increased erlotinib and OSI-420 accumulation in Saos2-BCRP cells $(p<0.01)$, and OSI-420 accumulation in LLCPK1-MDR1 cells ( $p$ $<0.001$ ) (Figure 2-8).

Furthermore, we sought to determine whether erlotinib is a substrate for influx transporters using HEK-293 cells transfected with specific uptake transporters. Uptake of erlotinib and OSI-420 was significantly higher in presence of organic anion transporter-3 (OAT3) $(p<0.001)$ as compared to the HEK-293 cells transfected with empty vector. Additionally, expression of the organic cation transporter-2 (OCT2) significantly 

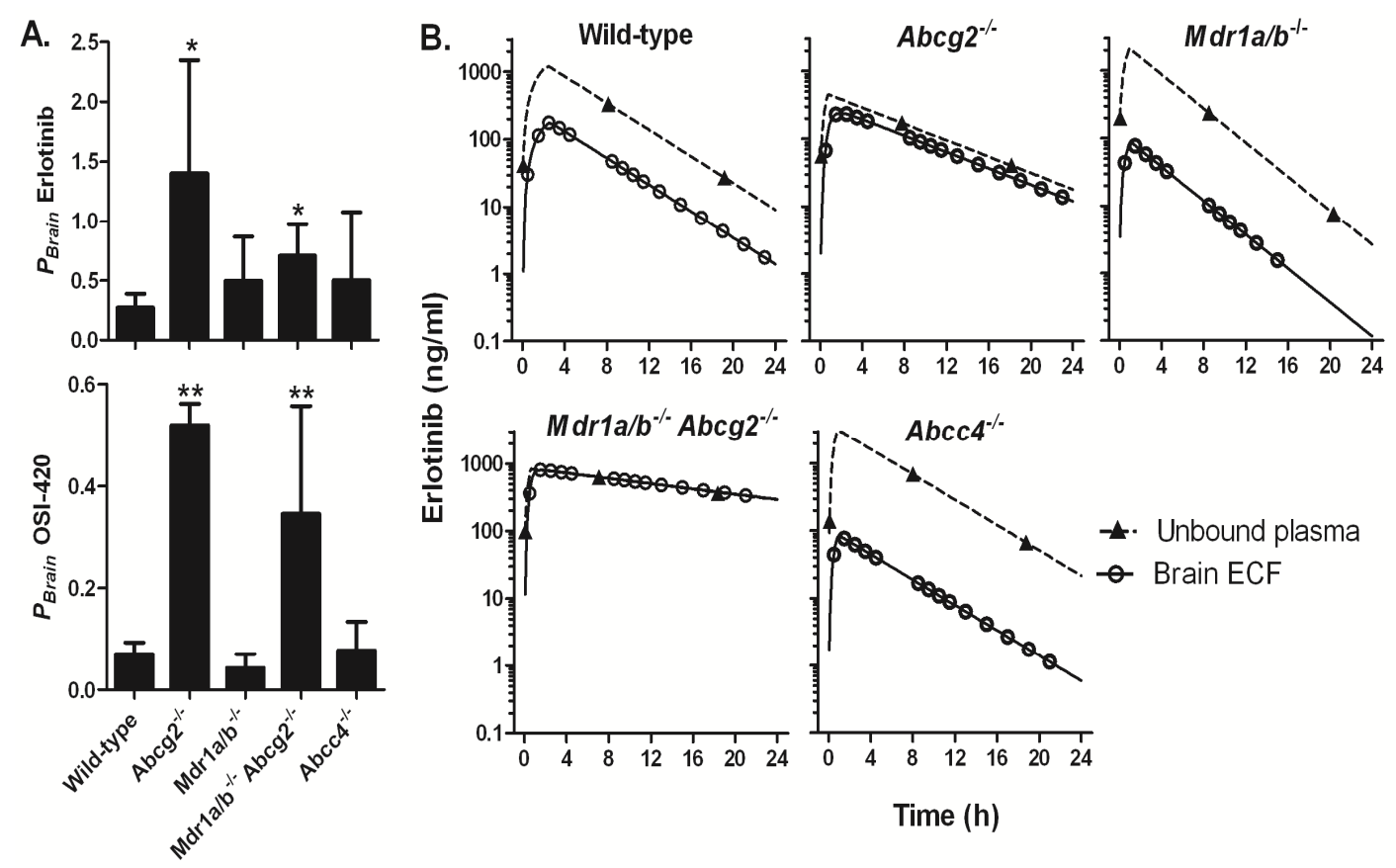

Figure 2-5. Brain penetration of erlotinib and OSI-420 after a single oral dose of erlotinib

A. Penetration of unbound erlotinib and OSI-420 to brain ECF expressed as $P_{\text {Brain }}$ (mean \pm SD from 4 to 7 mice). ${ }^{*}, P<0.05$ and $* *, P<0.01$, Mann-Whitney test of each knockout model compared with wild-type mice.

B. Representative unbound erlotinib concentration-time plots in brain ECF ( $\circ$ ) and plasma ( $\boldsymbol{\Delta}$ ) in wild-type, $A b c g 2^{-/-}, M d r 1 a / b^{-/-}, M d r l a / b^{-/-} A b c g 2^{-/-}$and $A b c c 4^{-/-}$mice. Model curves from individual predicted parameters are represented for plasma (dashed line) and brain ECF (solid line). 


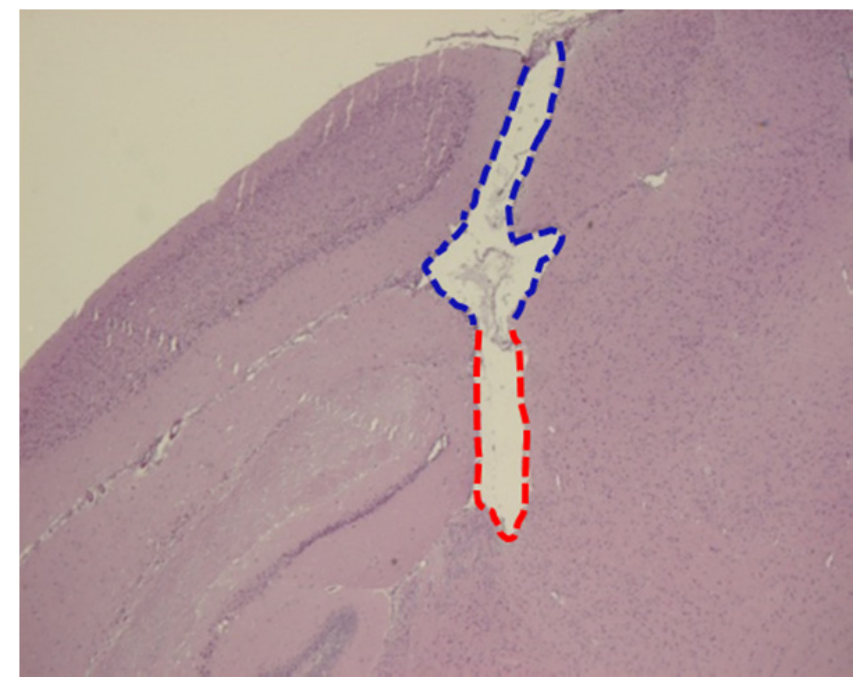

Figure 2-6. H\&E staining showing the microdialysis cannula and probe track in brain ECF with no gross pathological effect of $10 \%$ HPBCD on brain tissue

Blue dashes limit cannula track and red dashes limit probe track. 


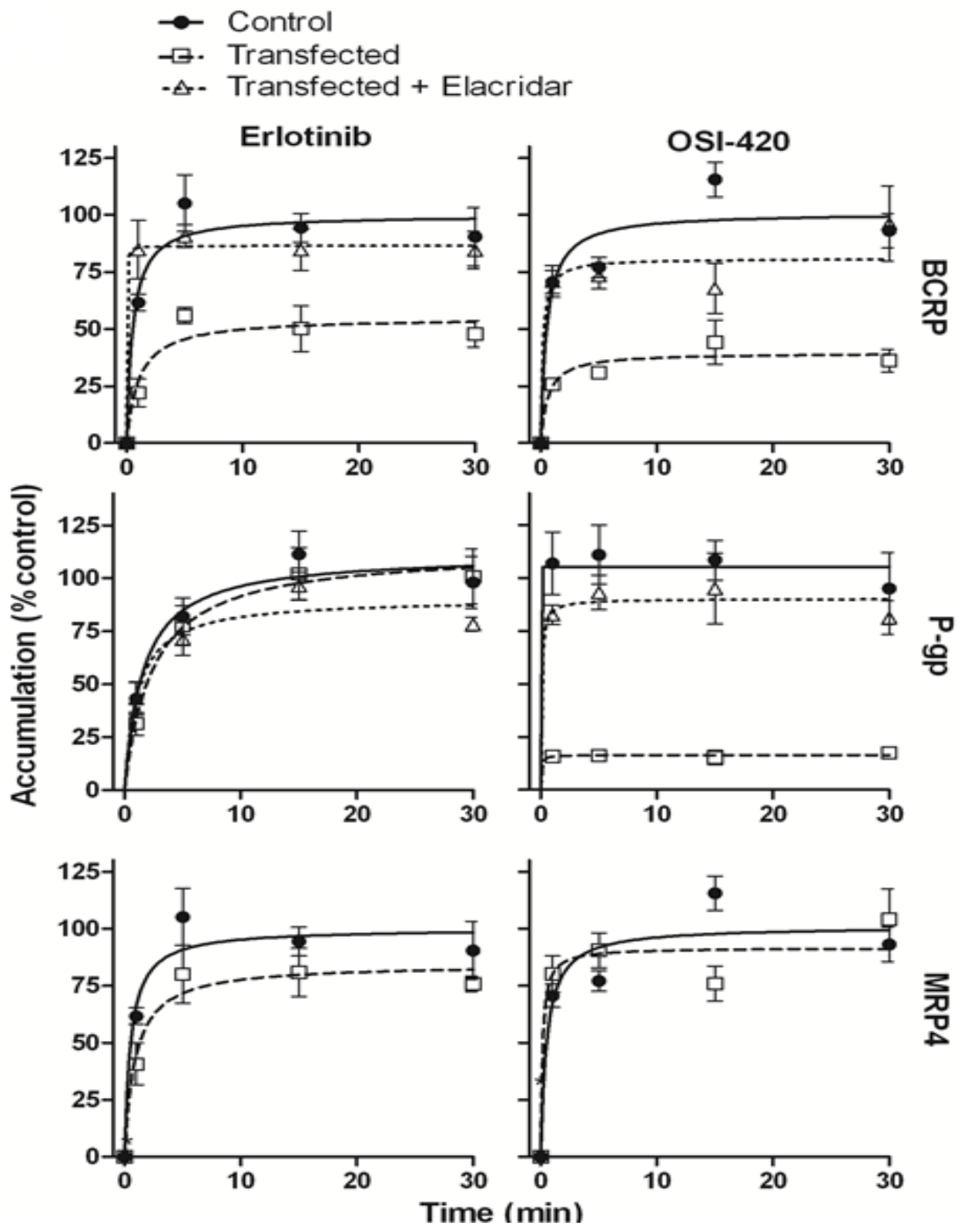

Figure 2-7. Erlotinib and OSI-420 intracellular accumulation in vitro in cell lines expressing efflux transporters

Time-course of drug accumulation in cell lines. Control, cells lines transfected with an empty vector control; Transfected, cell lines transfected with a vector expressing the indicated transporter; Transfected + Elacridar, transporter-transfected cells treated with 4 $\mu \mathrm{M}$ of the P-gp/BCRP inhibitor elacridar. Values are the percentage of the maximum accumulation (mean $\pm \mathrm{SD} ; \mathrm{n}=4-6$ ) in control cells (Saos2-pcDNA for BCRP and MRP4 or LLC-PK1 for P-gp). 


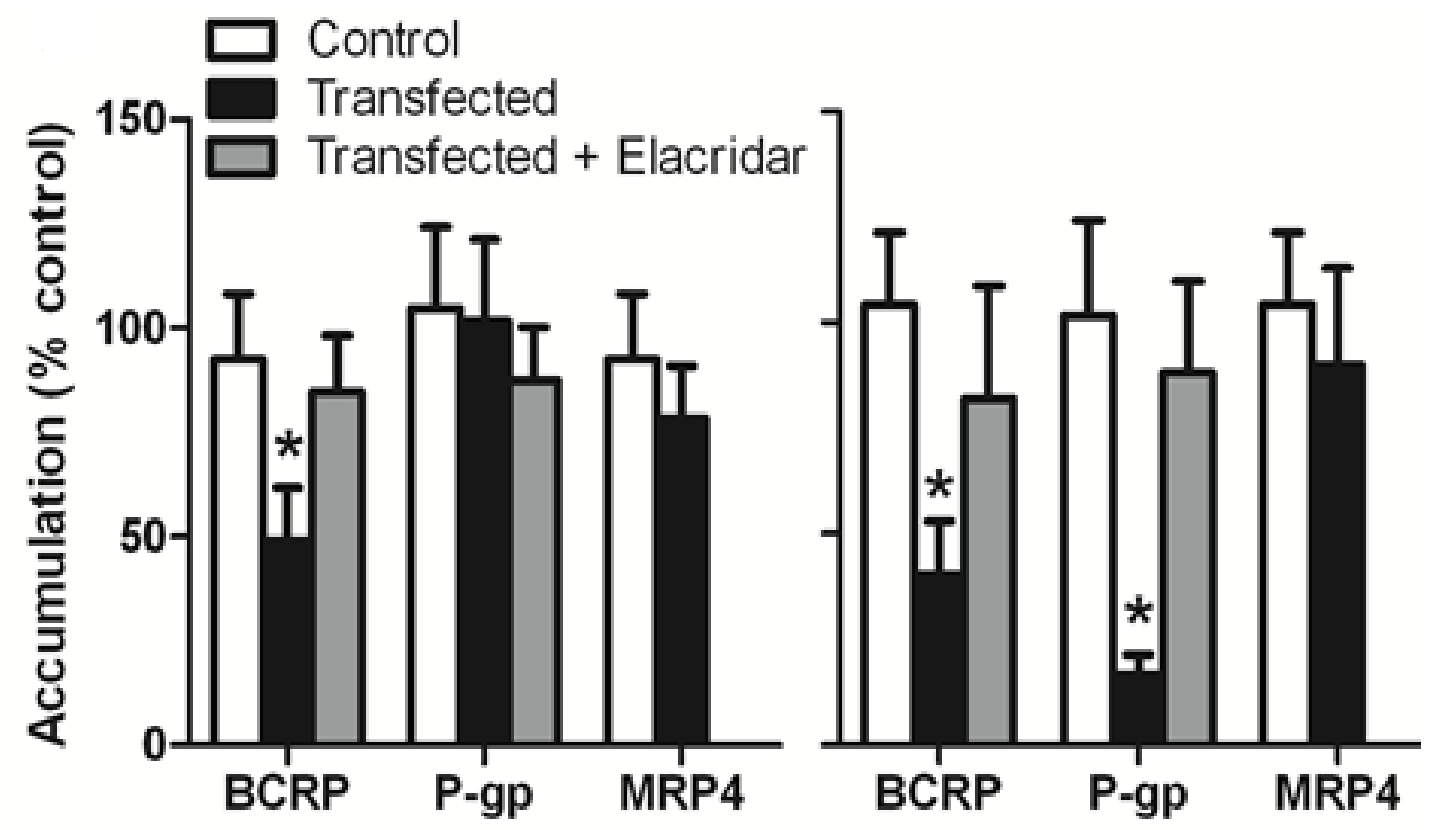

Figure 2-8. Intracellular accumulation data from $15 \mathrm{~min}$ and $30 \mathrm{~min}$ time-points combined

$* \mathrm{P}<0.001$ as compared to accumulation in control cells (ANOVA with posthoc Dunnet's test). 
increased erlotinib and OSI-420 uptake $(p<0.01)$ (Figure 2-9).

\subsection{DISCUSSION}

Using microdialysis sampling, transporter deficient mice, pharmacokinetic modeling, and cell lines transfected with efflux and uptake transporters, we elucidated the roles of transport mechanisms involved in erlotinib disposition in brain parenchyma. Our in vitro and in vivo data show that Bcrp1 is the primary efflux mechanism for erlotinib and OSI-420 at the murine BBB, whereas P-gp and Mrp4 have little or no effect on erlotinib CNS penetration. In vitro, intracellular accumulation of erlotinib and OSI-420 showed that only OSI-420, not erlotinib, accumulation was reduced in cells overexpressing MDR1. The present study also showed that erlotinib and OSI-420 are substrates for the human organic uptake transporter OAT3 and, to a lesser extent, OCT2.

Our technical approach of using microdialysis allowed the characterization of unbound (active) erlotinib concentrations in brain ECF. Our technique overcomes the limitations of (a) the CSF aspiration technique, which characterizes drug exposure in a very specialized CNS compartment (Shen et al., 2009), and (b) the whole brain homogenization technique, which does not discern between free and bound drug entities or between different CNS compartments (brain parenchyma, CSF, and brain vessels). Our study shows that the inclusion of HPBCD in the perfusate increases the recovery of erlotinib, probably by including the drug into the core of the HPBCD molecule (Khramov and Stenken, 1999b).

Although HPBCD proved indispensable for the detection of erlotinib and OSI-420 in the dialysate, its presence provided a challenge as we performed the recovery experiments. Because HPBCD forms inclusion complexes with erlotinib in the perfusate limiting drug movement to brain ECF, we could not use retrodialysis or no-net flux to estimate in vivo recovery values. Instead, we chose to use ZFR method to estimate recovery, realizing that as with all methods to calculate recovery, this approach has limitations. For example, the ZFR method should be applied when the target tissue (e.g., brain ECF) is at a steady state. Our initial microdialysis experiments $(n=4)$ conducted under constant flow rate of $0.5 \mu \mathrm{L} / \mathrm{min}$ showed that brain ECF concentrations dropped by only $\sim 12 \%$ over the 5 to 8 hour period. Thus, we assessed recovery during a period 5 to 8 hours after the erlotinib dose was administered.

Because we calculated the recovery during this time interval (i.e., 5 to 8 hours), we were only able to collect a single sample per flow rate. Although this short time frame may not have allowed adequate time to achieve stable recoveries at each flow rate, comparing in vitro recovery values obtained with either changing the flow rate or under constant (i.e., stable) flow rate yielded similar recovery values suggesting that stable recoveries can be achieved with our experimental approach (Table 2-3).

Our study has established that penetration of unbound erlotinib from plasma 


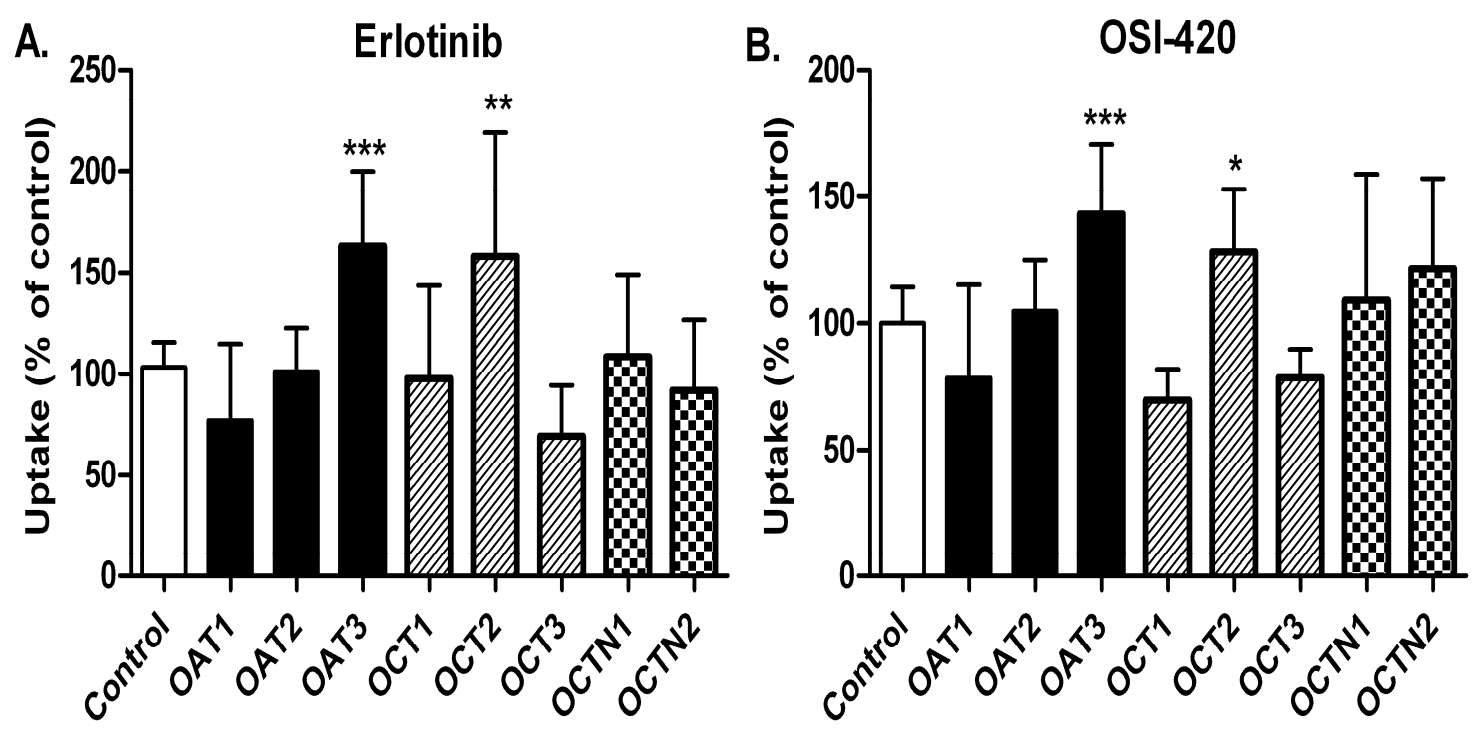

Figure 2-9. Transport of erlotinib, A and OSI-420, B by human organic ion transporters

Results are shown for drug accumulation in HEK293 cells after 5 minutes incubation in each cell line. Columns represent (mean \pm SD) 8 to 18 observations per expressed transporter, and are expressed as percentage of their respective control (white bar). Only one control bar is shown for clarity purposes. The contribution of each transporter towards erlotinib or OSI-420 uptake was established by comparing data obtained in HEK293 cells overexpressing the transporter and HEK293 cells transfected with an empty vector. $* \mathrm{P}<0.05$; ** $\mathrm{P}<0.01$; *** $\mathrm{P}<0.001$ versus control, one-way ANOVA was performed followed by a post Dunnett test. 
Table 2-3. In vitro recovery values (\%) obtained while changing perfusion flow rates in a similar fashion to in vivo experiment to recoveries obtained with stable flow rate

\begin{tabular}{ccc}
\hline $\begin{array}{c}\text { Flow rate } \\
(\boldsymbol{\mu l} / \mathbf{m i n})\end{array}$ & $\begin{array}{c}\text { Recovery }(\%) \text { with changing flow } \\
\text { rate } \\
(\text { mean } \pm \text { SD of } 5 \text { experiments) }\end{array}$ & $\begin{array}{c}\text { Recovery (\%) with constant } \\
\text { flow rate } \\
\text { (mean } \pm \text { SD of 4 experiments) }\end{array}$ \\
\hline 0.2 & $28.1 \pm 2.2$ & $32 \pm 3$ \\
0.5 & $16.2 \pm 1.9$ & $18.5 \pm 2$ \\
1 & $5.36 \pm 2.7$ & $7.3 \pm 1.2$ \\
4 & $4.8 \pm 1.4$ & $4.2 \pm 0.7$ \\
\hline
\end{tabular}

Similar recovery values shows ability to achieve stable recoveries during the ZFR recovery estimation in vivo. 
across the BBB of wild type mice is limited (27\%) and even lower for OSI-420 (7\%). Thus, even though OSI-420 exhibits anti-tumor activity (Meany et al., 2008; Moyer et al., $1997)$, its low CNS penetration and systemic exposure ( $10-20 \%$ that of erlotinib in humans) suggest a minor role for OSI-420 in erlotinib clinical activity in brain tumors (Broniscer et al., 2007b; Ling et al., 2006).

Our data show that Bcrp1 is the major efflux transporter limiting penetration of erlotinib and OSI-420 into murine brain ECF. In vitro studies showed reduced erlotinib and OSI-420 accumulation in Saos2-BCRP cells. Interestingly, a single nucleotide polymorphism in the $A B C G 2$ promoter that correlates with lower BCRP expression was associated with higher erlotinib plasma exposure, indicating the important role of BCRP in erlotinib disposition (Rudin et al., 2008).

Our results contrast with the conclusions by Kodaira et al stating that P-gp is the major efflux transporter for erlotinib at the murine BBB (Kodaira et al., 2010). This discrepancy can be explained by the different experimental designs used in each study. In the latter study, the authors administered erlotinib via the jugular vein for 2 hours and then analyzed erlotinib concentration in brain homogenates. They compared erlotinib concentration in brain homogenates to that in plasma only at one time point (i.e., 2 hours), and did not account for erlotinib plasma protein binding. This study design has several limitations. First, the homogenate technique could provide different results as it describes drug accumulation in the whole brain rather than in specific compartments, and second, relying on only one time point to determine the penetration ratio can mask the whole exposure profile for erlotinib in plasma as it provides only a "snap shot" of the penetration profile. In our study, we used the oral route for administering erlotinib to mimic the clinical situation. Our studies also took into consideration the plasma protein binding of both erlotinib and OSI-420.

While intracellular accumulation experiments showed that OSI-420 is a P-gp substrate, OSI-420 brain penetration was not increased in $M d r l a / b^{-/-}$mice. This can be due to the compensatory upregulation of Bcrp1 in $M d r 1 a / b^{-/-}$mice resulting in increased overall drug efflux from the brain. Our group previously used semi-quantitative immunohistochemical analysis to show that Bcrp1 is expressed at a higher level in the brain of Mdrla/ $b^{-/}$mice than that in wild-type mice (Shen et al., 2009). This observation agrees with previous study that shows 3 fold higher Bcrp 1 mRNA in $M d r 1 a / b^{-/-}$mice microvessels compared to wild-type mice (Cisternino et al., 2004).

In vitro screening of uptake transporters using HEK-293 transfected cells identified erlotinib and OSI-420 as substrates for OAT3 and OCT2. The affinity of these transporters for both anionic and cationic molecules has been previously reported (Ahn et al., 2009; Arndt et al., 2001). While HEK-293/OCT2 cells showed significantly higher erlotinib uptake, OCT2 contribution towards erlotinib uptake should be cautiously interpreted given the $\sim 40$ fold higher expression of $S L C 22 A 2$ gene, encoding OCT2, in these cells (Filipski et al., 2008). Studying erlotinib disposition in Oct2-deficient mice will clarify the affinity of OCT2 towards erlotinib. 
Our results could have clinical significance as Oat3 localized at the apical border of the choroid plexus can restrict the penetration of its substrates in the CSF (Nagata et al., 2002). Our group previously demonstrated that Bcrp1 and P-gp, localized apically at the choroid plexus, pump their substrate, topotecan, in the CSF (Shen et al., 2009; Zhuang et al., 2006). Thus, Oat 3 can oppose the transport direction of P-gp and Bcrp1 at the choroid plexus decreasing CSF drug accumulation. In rodents, Oat3 is predominantly expressed at the basolateral border of brain endothelial cells where it has been implicated in the brain to blood transport of its substrates (Kikuchi et al., 2003; Mori et al., 2003). Further, OCT2 is expressed at the apical border of the endothelial cells and was shown to increase the brain accumulation of their substrates (Lin et al., 2010).

Thus, in absence of Bcrp1 (i.e., in $\mathrm{Abcg} 2^{-/-}$or $\mathrm{Mdrla} / \mathrm{b}^{-/-} \mathrm{Abcg} 2^{-/-}$mice), the net erlotinib ECF accumulation would be determined by the net effect of factors that favor or oppose brain ECF penetration. Factors that favor erlotinib accumulation in the ECF include OCT2 at the BBB and lack of Bcrp1 at the BBB (Figure 2-10). On the other hand, Oat3 at the BBB and P-gp at the choroid plexus may restrict erlotinib accumulation in brain ECF by moving the drug from the brain to the blood or the CSF as schematized in Figure 2-10. This may explain the more than unity ECF accumulation for $\mathrm{ABC}$ transporters substrates in $\mathrm{Abcg} 2^{-/-}$and $\mathrm{Mdrla} / \mathrm{b}^{-/-} \mathrm{Abcg} 2^{-/-}$mice observed in our study and others (Kodaira et al., 2010; Kusuhara and Sugiyama, 2005; Lassman et al., 2005). Another transporter of potential interest is the organic anion transporting polypeptide (OATP2). OATP2 is expressed on both the apical and basolateral membranes of brain endothelial cells and probably mediates the accumulation of its substrates across the intact BBB vessels (Bronger et al., 2005; Gao et al., 1999; Kusuhara and Sugiyama, 2005). Further studies are warranted to identify the role of uptake transporters in erlotinib CNS penetration.

Regarding the implications of our results on erlotinib elimination, OCT2 and OAT3 transporters are abundantly expressed in the kidney (Bleasby et al., 2006). These transporters may explain the renal clearance component of erlotinib elimination, which accounts for $\sim 10 \%$ of the overall elimination (Ling et al., 2006). However, transporters expressed in the liver such as OAT2 and OCT1 were not found to transport erlotinib indicating the involvement of other transport mechanisms such as OATP1B1 and OAT1B3.

In conclusion, erlotinib is a Bcrp1 substrate whereas its metabolite is a substrate for both P-gp and Bcrp1. Erlotinib brain ECF accumulation was restricted mainly by Bcrp1. Intracellular accumulation studies confirmed the role of BCRP in erlotinib efflux and the dual P-gp/BCRP inhibitor, elacridar, increased erlotinib accumulation in cells in vitro. This study also indicates that erlotinib and OSI-420 are substrates for the uptake transporters OAT3 and OCT2. Further study is warranted to assess the role of these transporters in erlotinib brain accumulation. Our future experiments will focus on studying the effect of $\mathrm{ABC}$ transporter inhibitors on erlotinib penetration in high-grade glioma using genetically engineered animal models that recapitulate the genetics, the biology, and the histology of human tumors. 

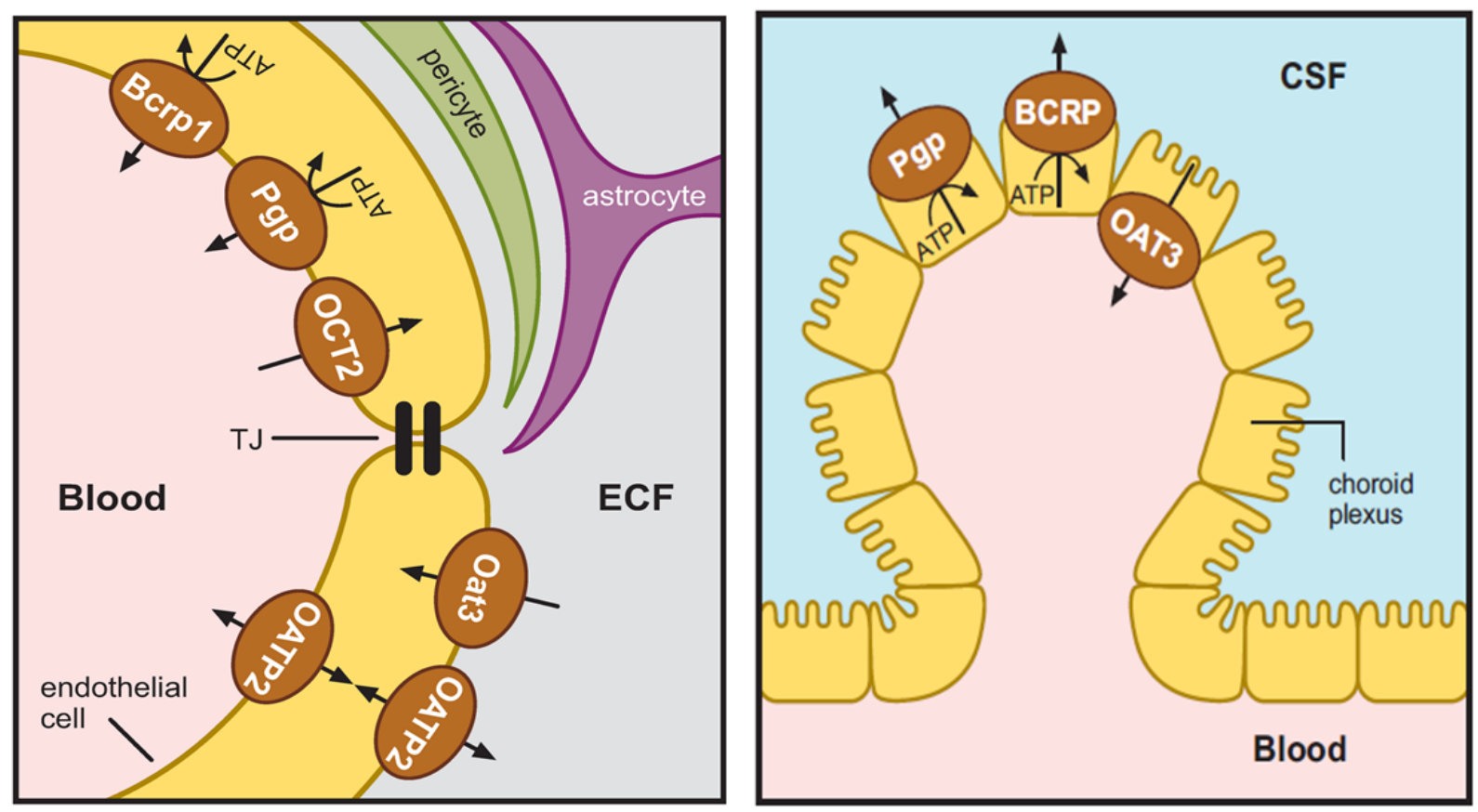

Figure 2-10. Proposed model for the role of efflux and uptake transporters in erlotinib CNS penetration

A. Schematic diagram for endothelial cells with transporters localized on either apical border (facing blood) or basolateral border (facing brain ECF).

B. Schematic diagram for the choroid plexus forming the BCSFB. Transporters are localized on either the apical border (facing $\mathrm{CSF}$ ) or basolateral border (facing blood). The arrows represent the direction of drug transporter. 


\section{CHAPTER 3. MAGNETIC RESONANCE IMAGING (MRI)-GUIDED MICRODIALYSIS CANNULA IMPLANTATION IN A SPONTANEOUS HIGH-GRADE GLIOMA MURINE MODEL*}

\subsection{INTRODUCTION}

Cerebral microdialysis sampling is used to monitor CNS anti-cancer drug disposition in mouse models (Boschi and Scherrmann, 2000). This approach provides advantages over other sampling methods (e.g., whole tissue homogenization) including sampling from discrete anatomic compartments such as the brain extracellular fluid (ECF) or ventricular cerebrospinal fluid (vCSF) (Elmeliegy et al., 2011b; Shen et al., 2009), sampling from normal brain or brain tumor tissue, and acquisition of unbound or pharmacologically active drug moieties(Chaurasia et al., 2007). Furthermore, microdialysis enables serial sampling from individual animals, thereby reducing the number of animals required for pharmacokinetic investigations (Pan et al., 2007).

Microdialysis has been frequently employed in studying anti-cancer drug penetration within brain tumors, particularly high-grade gliomas (Blakeley et al., 2009; Carcaboso et al., 2010; Ma et al., 2003; Ma et al., 2001). These studies are most often performed using murine orthotopic xenograft models in which human glioma cells are stereotactically injected into a defined brain location and the microdialysis guide cannula is simultaneously implanted. Tumor tissue then develops and surrounds the cannula (Carcaboso et al., 2010; Ma et al., 2003; Ma et al., 2001).

Although orthotopic tumor xenograft models are easy to use, relatively inexpensive, and reproducible, in many cases the genetics and histology of human tumors are not adequately recapitulated (Richmond and Su, 2008). In addition, the tumor microenvironment and host immune responses are likely altered in immune compromised mice, which diminish the ability of xenograft models to truly recapitulate the features observed in human tumors (Becher and Holland, 2006).

In contrast, genetically engineered murine models (GEMMs) of brain tumors bestow many of the genetic and histological features of malignant human brain tumors (Becher and Holland, 2006). In general, these mouse models develop tumors spontaneously upon alteration of signaling pathways critical for the development of the specific tumor of interest (Becher and Holland, 2006; de Vries et al., 2009). Thus, molecular and other complex processes including specific contributions of the tissue microenvironment, such as tumor angiogenesis, can appropriately mimic human disease in these spontaneous tumor models.

*Reprinted with permission. Elmeliegy MA et al. (2011) Magnetic resonance imagingguided microdialysis cannula implantation in a spontaneous high-grade glioma murine model. J Pharm Sci 100:4210-4214. 
In GEMMs, heterogeneous tumor growth patterns and development of tumors in different brain regions pose several technical challenges to using these models for CNS pharmacokinetic investigations (de Vries et al., 2009). For example, a major challenge to using cerebral microdialysis to study anti-cancer drug penetration in tumors of GEMMs is the difficulty of acquiring stereotactic coordinates to accurately place a microdialysis cannula in the tumor. Traditionally, the coordinates of the intersection of the coronal and sagittal sutures (bregma point) are used as a reference point for the placement of the microdialysis cannula (Bert et al., 2004; Shen et al., 2009). However, this approach is not practical in GEMMs because the bregma does not appear on images derived by MRI, which is the imaging method used to identify the size and location of the spontaneously arising tumors in the brain.

The objective of the current study was to use MRI imaging to identify a reference point for implanting microdialysis cannula in spontaneously arising tumors in a GEMM for high-grade glioma. The accuracy of cannula placement was verified by post-mortem MRI and histological examination.

\subsection{MATERIALS AND METHODS}

\subsubsection{Animals}

Transgenic mice used in this study were previously described (Chow et al., 2011). Briefly, conditional deletion of Pten, Tp53, and Rb1 in Gfap-expressing cells was induced by I.P injection of tamoxifen (Sigma, St. Louis, MO) to GFAP-CreER ${ }^{T M}$; $P t e n^{\text {flox/flox }} ; T p 53^{\text {flox/flox }} ; R b 1^{\text {flox/flox }}$ post-natal day 30 (P30) mice at a dosage of $9 \mathrm{mg} / 40 \mathrm{gm}$ weight daily for 3 consecutive days. Mice were maintained on a $12 \mathrm{~h}$ light/dark cycle with free access to food and water. Mice developed tumors within 4 to 6 months. Other mouse strains (C57BL6 and CD1 athymic nude mice) were also used for validation of reference point studies. All animal studies were carried out in compliance with the Animal Care and Use Committee at St. Jude Children's Research Hospital.

\subsubsection{Magnetic resonance angiography (MRA)}

MRA data were produced from a Bruker FL2D Time-of-Flight (TOF) protocol $\left(\mathrm{T}_{\mathrm{R}} 7.6 \mathrm{~ms} ; \mathrm{T}_{\mathrm{E}} 3.77 \mathrm{~ms}\right.$; Flip angle 90 degree). The raw data were acquired in the coronal plane followed by Maximum-Intensity Projection (MIP) reconstruction on a Siemens workstation using Syngo MR B15 software (Siemens, Erlangen, Germany). Image processing procedures were similar for both angiography and post-mortem MRI studies. 


\subsubsection{Magnetic resonance imaging (MRI)}

MRI exams were performed using a 7-Tesla Bruker Clinscan animal MRI scanner and a 4-channel phased-array surface coil (Bruker BioSpin MRI GmbH, Germany). Animals were anesthetized using isoflurane during data acquisition. Turbo Spin Echo protocols ( $T_{R} 2000-2500 \mathrm{~ms} ; T_{E} 40 \mathrm{~ms}$ ) were used to produce $T 2$ weighted transverse images with slice thickness of $0.5 \mathrm{~mm}$. Turbo Gradient Spin Echo protocols (TR 2000 $\mathrm{ms}$; TE $40 \mathrm{~ms}$; Flip angle 180 degree) were used to produce fast T2-weighted images in the coronal plane with slice thicknesses of $0.7 \mathrm{~mm}$. For post-mortem MRI, the mouse heads were submerged in Fluorinert FC-770 liquid (3M, Belgium) and imaged by a TSE protocol with a rat head 4-channel phased-array surface coil.

\subsubsection{Stereotactic surgery for microdialysis cannula insertion}

The general surgical procedure has been previously described (Leggas et al., 2004b). Briefly, a mouse was anesthetized with $50 \mathrm{mg} / \mathrm{kg}$ ketamine (Hospira, Inc., Lake Forest, IL) and $10 \mathrm{mg} / \mathrm{kg}$ xylazine (VEDCO, St. Joseph, MO). The head of the mouse was fixed in position on a KOPF stereotactic apparatus (Tujunga, CA). A small incision on the scalp was made and the rostral rhinal vein was identified.

Using MRI images, tumor coordinates were calculated using View-Dimensions tool in Syngo MR B15 software. A microdialysis guide cannula was implanted in the center of a tumor. The mouse was allowed to recover for 3-5 days. On the day of the microdialysis study, a microdialysis probe (MBR-1-5, Bioanalytical Systems) was inserted through the cannula. The probe was perfused with artificial cerebrospinal fluid (aCSF, Leggas et al., 2004b) at $0.5 \mu \mathrm{L} / \mathrm{min}$, and the probe membrane was allowed to equilibrate for 1 hour before starting the microdialysis experiment. Microdialysis experiments were performed as previously described (Elmeliegy et al., 2011b).

\subsubsection{Histology}

At the end of the microdialysis experiment, the animal was euthanized and the brain was removed and fixed in $10 \%$ neutral buffered formalin for at least $24 \mathrm{hr}$. Cannula and probe were removed and the brain was sliced into sagittal sections around the location of the implanted cannula, dehydrated, cleared, and embedded in a paraffin block. Sections were cut on a microtome at $4 \mu \mathrm{m}$ thickness and every tenth section was collected and stained with haematoxylin and eosin. All sections from a given block were examined microscopically to verify the accurate placement of the microdialysis probe via the track in the tumor. 


\subsection{RESULTS AND DISCUSSION}

\subsubsection{Identification of the rostral rhinal vein as a correlative reference point}

Images of blood vessels of the mouse brain acquired using angiography revealed the presence of a large vein overlying the border between the olfactory bulb and the frontal lobe (Figure 3-1A and B). Using the mouse cerebral vasculature atlas (Dorr et al., 2007), this vein was identified as the rostral rhinal vein, which is readily apparent to the unaided eye and traverses both the dorsal and ventral surfaces following the olfactory bulb/frontal lobe border (Figure 3-1C). The removal of skulls ( $\mathrm{n} \geq 6$ per strain used) confirmed that this vein overlies the border between the olfactory bulb and the frontal lobe. Thus, we designated our reference point as the intersection of the midline suture and the rostral rhinal vein on the mouse brain surface. This point correlated with the intersection between the midline and the olfactory bulb/frontal lobe border visualized on T2-weighted MRI images of the brain. The rostral rhinal vein was present in all mouse strains evaluated including C57BL6 and CD1 athymic nude. Anatomical dissection of these strains confirmed that this vein overlies the olfactory bulb/frontal lobe border, thus this method may be applied to target tumors in other mouse strains.

\subsubsection{Determination of tumor coordinates and confirmation of probe placement}

Tamoxifen injection induced Cre activity and spontaneous glioma formation in

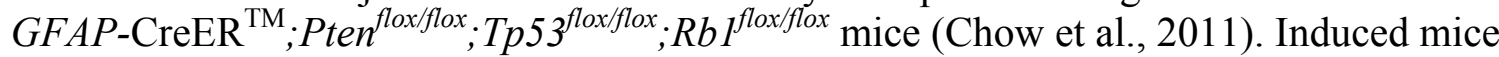
were monitored biweekly for tumor development by MRI using T2-weighted imaging beginning at P120. Tumors developed in several locations within the brain including the forebrain, midbrain, and the cerebellum. When a tumor was large enough for microdialysis cannula/probe implantation (around 2.5-4 $\mathrm{mm}$ in diameter), placement coordinates were determined using the View-Dimensions tool in Syngo MR B15 software. The $X$ and $Y$ axes were determined using the transverse plane (Figure 3-2A) while the depth of implantation (Z-axis) was determined using the coronal plane (Figure 3-2B). Post-mortem MRI (Figure 3-2C and D) and immunohistochemical examination (Figure 3-2E) confirmed targeted probe placement in all mice studied $(n=6)$. Mice used in this study developed tumors that arose within several regions of the brain including the forebrain (33\%), midbrain (50\%), and the cerebellum (17\%).

\subsection{CONCLUSION}

It is a challenge to accurately place microdialysis cannulae in GEMMs that spontaneously develop brain tumors because the traditional reference point commonly used to derive stereotactic co-ordinates for cannula implantation, the bregma, is not visible by MRI. By using a combination of angiography and T2-weighted imaging by MRI, we identified the rostral rhinal vein as a reference point to derive coordinates for accurate placement of microdialysis cannula in tumors developing in several regions of 

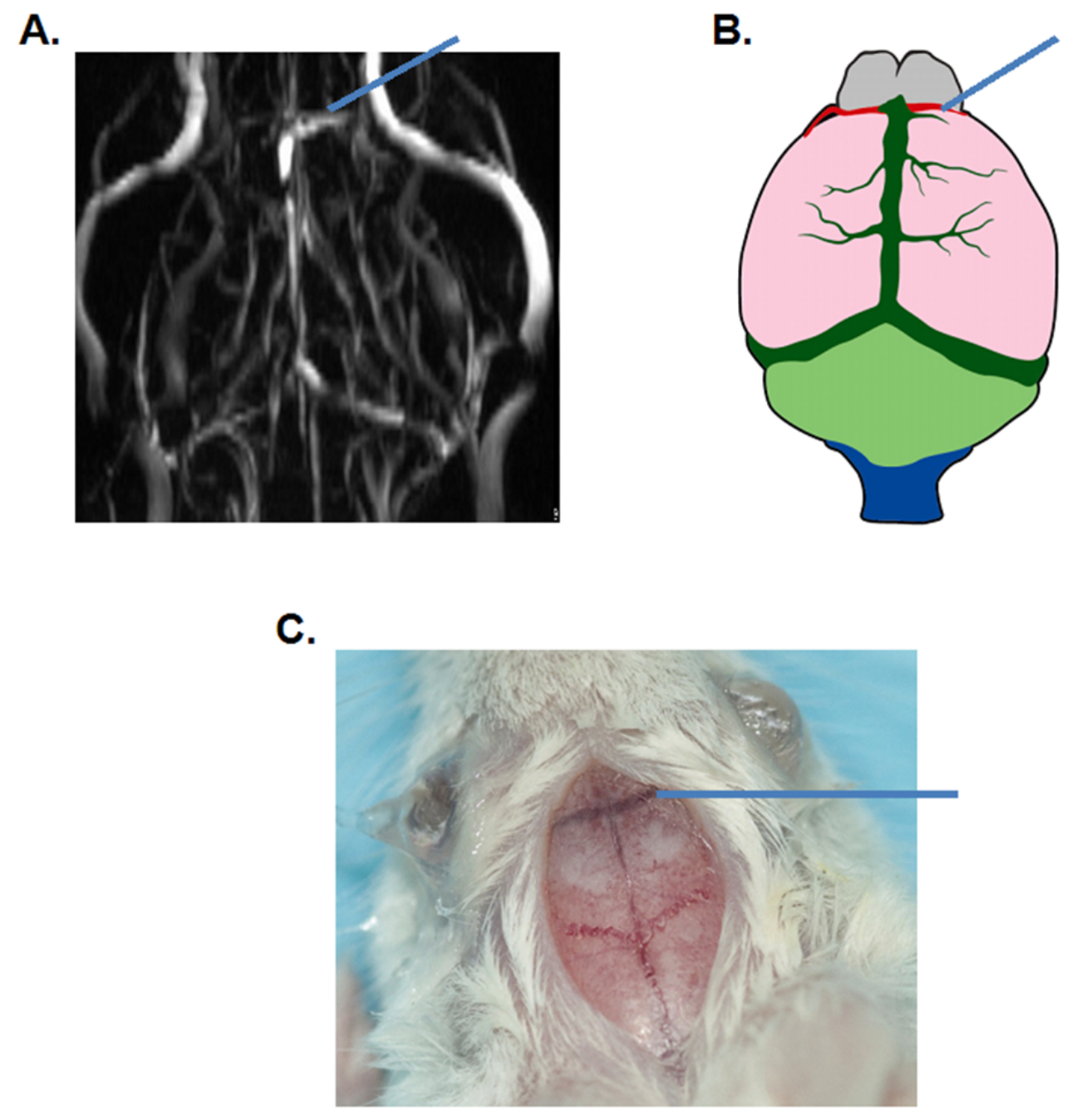

Figure 3-1. Presence of the rostral rhinal vein on the surface of a mouse brain

A. Angiogram of a non-tumor bearing GFAP-CreER $R^{T M}$; ten $^{\text {flox/flox }} ; \mathrm{T} p 53^{\text {flox/flox }}$;
$R b 1^{\text {floxflox }}$ mouse showing a large vein across the olfactory bulb/frontal lobe border.

B. Diagrammatic illustration of veins on mouse brain surface illustrating the rostral rhinal vein on the border between the olfactory bulb and the mouse brain frontal lobe. Reproduced with permission from (Dorr et al., 2007).

C. Brain surface of a GFAP-CreER $R^{T M} ; \operatorname{Pten}^{\text {flox/flox }} ; \mathrm{T} p 53^{\text {flox/flox }} ; R b 1^{\text {flox flox }}$ mouse. Lines indicate location of the rostral rhinal vein. 
Figure 3-2. Derivation of MRI-guided stereotactic coordinates for cannula implantation in spontaneously arising brain tumors

A. Transverse section on a T2-weighted MRI demonstrating a tumor in the left hippocampus. Tumor margins are enclosed by a dashed line. The intersection between the olfactory bulb/frontal lobe border and the midbrain was used as a reference point to determine the coordinates on the $\mathrm{Y}$-axis (a) and the $\mathrm{X}$-axis (b).

B. Coronal section on a T2-weighted MRI for calculation of the depth coordinates, i.e. $\mathrm{Z}$-axis $(c)$. In the provided example, values for $\mathrm{a}, \mathrm{b}$, and $\mathrm{c}$ were $7.6,3$, and $1.3 \mathrm{~mm}$, respectively.

$\mathrm{C}$ and D. Post-mortem MRI showing track of the microdialysis probe in the tumor in the $\mathrm{X}$ and $\mathrm{Y}$ axes (red arrow), and on the $\mathrm{Z}$-axis (yellow arrow).

E. Hematoxylin and eosin staining confirming probe location in the targeted area (yellow dots limit tumor margins, blue dashes limit cannula track, and red dashes limit probe track; lines were drawn with Adobe Photoshop V11.0). 
A.

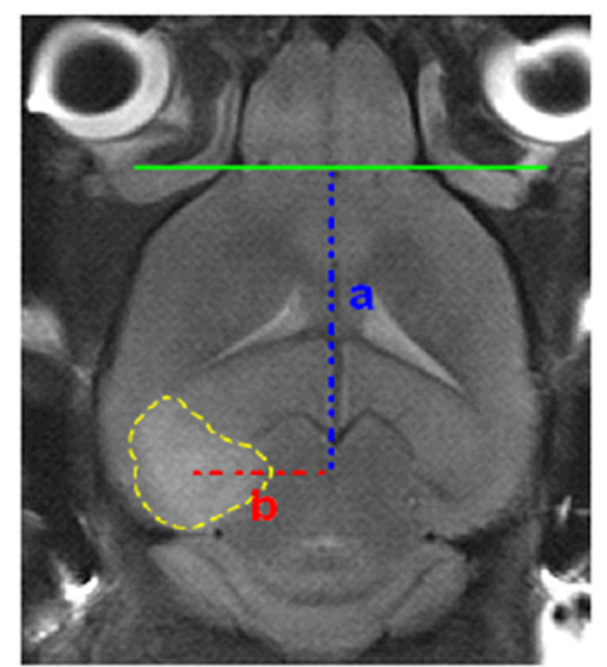

c.

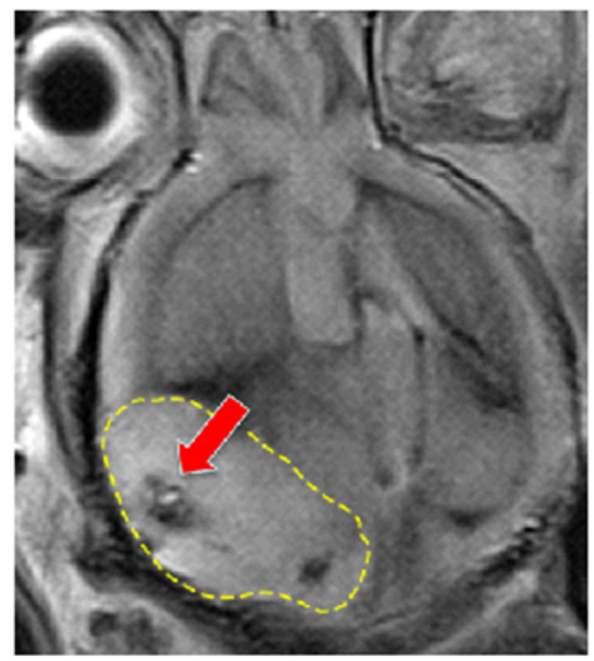

E.

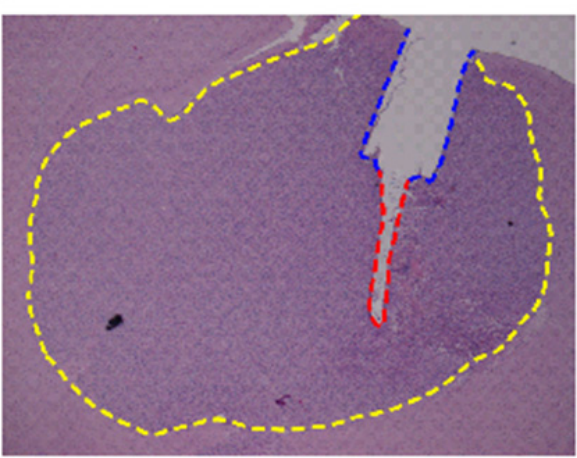

B.

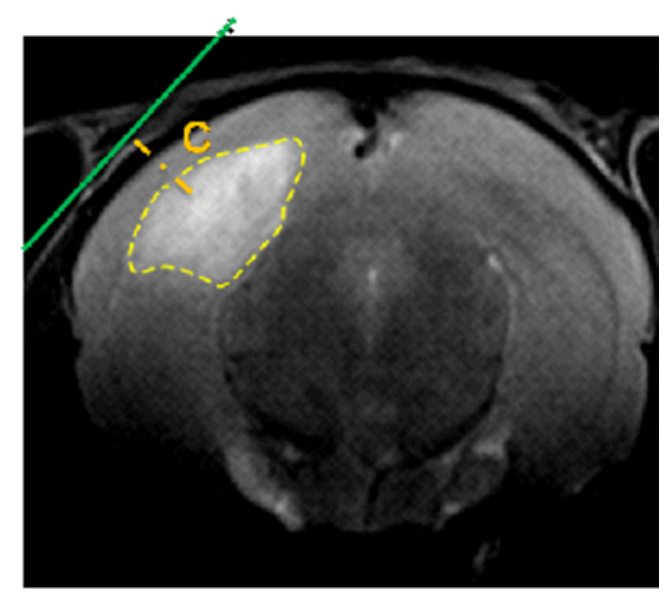

D.

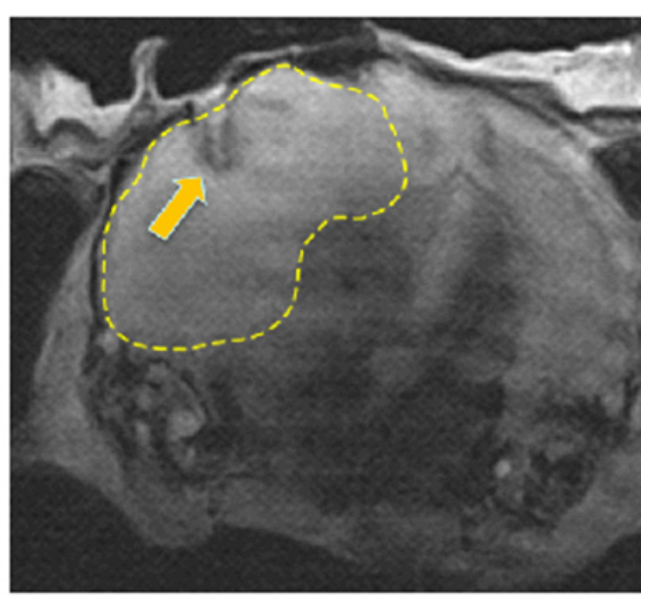


the mouse brain. 


\section{CHAPTER 4. CEREBRAL MICRODIALYSIS FOR SIMULTANEOUS SAMPLING OF TWO TYROSINE KINASE INHIBITORS IN AN ORTHOTOPIC MOUSE MODEL OF GLIOMA}

\subsection{INTRODUCTION}

Anti-cancer drug penetration within brain tumors can be assessed using different strategies including cerebral microdialysis (de Lange et al., 2000). This method provides several advantages over brain tissue or tumor homogenization, including a significant reduction in the number of animals required per study due to collection of multiple samples per subject. Other advantages include providing compartment-specific information about drug penetration and sampling of the unbound (pharmacologically active) form of the drug. Cerebral microdialysis involves sampling of the target tissue ECF (e.g., brain parenchyma, ventricular CSF, or tumor) via a semi-permeable probe (Brunner and Langer, 2006). Perfusing the probe with a solution that mimics the composition of the tissue ECF (i.e. perfusate) allows small molecules (e.g., anti-cancer drugs) in the tissue ECF to diffuse across the semi-permeable membrane down their concentration gradient. The collected samples (i.e. dialysates) can then be measured using various analytical methods.

Microdialysis is commonly used to sample tissue ECF to assess the disposition of a single anti-cancer agent per experiment. However, emerging therapies for brain tumors include combining more than one targeted agent due to the highly resistant nature of some cerebral tumors such as malignant glioma (Fan and Weiss, 2010; Karpel-Massler et al., 2011; Krakstad and Chekenya, 2010). Thus, a robust method to sample multiple anticancer agents simultaneously coupled with adequate analytical methods can prove useful in determining the penetration profile of multiple anti-cancer agents administered in combination. To our knowledge, the technical feasibility of simultaneous sampling of multiple anti-cancer agents via cerebral microdialysis has not yet been published.

One major limitation of microdialysis is the difficulty of sampling lipophilic drugs (Benfeldt and Groth, 1998; Carneheim and Stahle, 1991). Such compounds are generally highly-protein bound and thus only a small fraction of these compounds is available for recovery through the microdialysis probe (Benfeldt and Groth, 1998). Lipophilic compounds also tend to adhere to the polymeric materials of the microdialysis probe and tubing. Such poor recovery represents a challenge against the use of microdialysis to study the disposition of these lipophilic agents (de Lange et al., 2000). Attempts to enhance recovery of lipophilic compounds involved adding BSA in the perfusate, which reduces drug adsorption to microdialysis tubings (Carneheim and Stahle, 1991; Jensen et al., 2007). Despite the enhanced recovery, adding BSA can cause some analytical challenges in sample cleanup for drug analysis using liquid chromatography (Stenken et al., 2001).

The use of HPBCD has been proposed as an alternative to BSA to enhance the recovery of lipophilic drugs (Duo et al., 2006; Khramov and Stenken, 1999a; b). We 
previously reported improved recovery of erlotinib upon including $10 \%$ HPBCD in the perfusate (Elmeliegy et al., 2011b). In the present study, we tested whether addition of this compound could improve the recovery of another tyrosine kinase inhibitor, crenolanib (CP-868,596; AROG Pharmaceuticals). Crenolanib is an investigational drug that specifically and potently inhibits PDGFR $\alpha$ and PDGFR $\beta$ (Lewis et al., 2009). Currently, crenolanib is undergoing phase I trials for patients with either diffuse intrinsic pontine glioma (DIPG) or recurrent high-grade glioma (HGG). Understanding the penetration of crenolanib as a single agent and in combination with another tyrosine kinase inhibitor, erlotinib in murine models of glioma will provide further insights into the best use of these agents in regimens for treatment of brain tumors.

We conducted in vitro experiments to determine the appropriate conditions to dialyze crenolanib and erlotinib, either alone or simultaneously. We then applied these conditions to study crenolanib and erlotinib penetration as single agents or when administered in combination in an orthotopic mouse model of glioblastoma.

\subsection{MATERIALS AND METHODS}

\subsubsection{Chemicals and reagents}

Erlotinib and OSI-420 analytical standards, and their deuterated internal standard used in the mass spectrometry assay were provided by OSI pharmaceuticals (Uniondale, NY). HPBCD was purchased from CTD, Inc. (High Springs, FL). Crenolanib (CP868, 596 freebase, and its deuterated internal standard were supplied by Pfizer, New London, Connecticut, USA).

\subsubsection{Liquid chromatography/tandem mass spectrometry (LC MS/MS) method for crenolanib determination in mouse plasma and CSF samples}

Crenolanib and its deuterated form as an internal standard (ISTD) were separated on a Pursuit $3 \mu$ Diphenyl column (Varian Inc., Lake Forest, CA; $50 \times 2.0 \mathrm{~mm}$ ) with a mobile phase consisting of acetonitrile $/ 10 \mathrm{mM}$ formic acid $=85 / 15 / 0.1$. Samples were processed using liquid-liquid extraction with t-butyl methyl ether. Extracted samples were dried by nitrogen gas and reconstituted with Acetonitrile/10mM formic acid = 60/40/0.1. Detection was achieved by an API-5500 LC-MS/MS system (Applied Biosystems, Carlsbad, California).

\subsubsection{In vitro probe recovery}

To evaluate probe recovery of crenolanib and erlotinib, stock solutions containing $30 \mathrm{ng} / \mathrm{mL}$ crenolanib, $30 \mathrm{ng} / \mathrm{mL}$ erlotinib, or $30 \mathrm{ng} / \mathrm{mL}$ of each in aCSF were prepared. A $1 \mathrm{~mm}$ microdialysis probe (MBR-1-5 brain probe, BASi, West Lafayette, IN) was 
inserted into each solution. The probe was perfused with: (a) aCSF or (b) $10 \% \mathrm{HPBCD} / \mathrm{aCSF}$ at $0.5,1,2$, and $4 \mu \mathrm{L} / \mathrm{min}$ flow rate. Drug concentrations in each set of dialysates and stock solution samples were analyzed using LC-MS/MS. Concentrations were plotted against flow rate and extrapolation to a hypothetical ZFR was performed as previously described (Menacherry et al., 1992) using non-linear regression analysis in GraphPad Prism (GraphPad Software, San Diego, CA) using the following formula:

$$
\mathrm{C}_{\text {Dial }}=\mathrm{C}_{0} * \exp (-\mathrm{rA} * \mathrm{X})
$$

where $\mathrm{r}$ (mass transport coefficient) and A (surface area of the dialysis membrane) are constants, estimated using non-linear regression, $\mathrm{C}_{0}$ represents drug concentrations in the dialysed tissue, $\mathrm{C}_{\text {Dial }}$ is drug concentration in dialysates, and $\mathrm{X}$ is the flow rate at which the dialysate was collected (Menacherry et al., 1992).

We then sought to determine whether the retrodialysis method, illustrated in Figure 1-5, could be used to estimate probe recovery in presence of an affinity-based trapping agent (HPBCD) in the perfusate. Thus, we perfused the probe with $30 \mathrm{ng} / \mathrm{mL}$ crenolanib in $10 \% \mathrm{HPBCD} / \mathrm{aCSF}$ at $0.5 \mu \mathrm{L} / \mathrm{min}$. The collected dialysate was then used to determine the percentage of drug lost from the probe according to the following equation:

$$
\operatorname{Drug} \operatorname{lost}(\%)=\frac{\text { Cperfused }- \text { Ccollected }}{\text { Cperfused }} \times 100
$$

where Cperfused is crenolanib concentration in the perfusing solution i.e. $30 \mathrm{ng} / \mathrm{mL}$ and Ccollected is crenolanib concentration measured in the collected dialysate.

On a separate experiment, we determined the percentage of drug gained from 30 $\mathrm{ng} / \mathrm{mL}$ crenolanib stock solution when perfused with $10 \% \mathrm{HPBCD} / \mathrm{aCSF}$ at $0.5 \mu \mathrm{L} / \mathrm{min}$ according to the following equation:

$$
\text { Drug gained }(\%)=\frac{\text { Cdial }}{\text { Cstock }} \times 100
$$

where Cdial is crenolanib concentration in the dialysate collected at $0.5 \mu \mathrm{L} / \mathrm{min}$ and Cstock is crenolanib concentration in the stock solution, i.e. $30 \mathrm{ng} / \mathrm{mL}$.

\subsubsection{Tumor lines}

Luciferase-labeled SJG2 glioma cell line was cultured in DMEM supplemented with 10\% fetal bovine serum from Invitrogen (Carlsbad, CA). This cell line was generated from a 10-year old female diagnosed with malignant glioma at St. Jude Children's Research Hospital (Memphis, TN). This tumor line has been modified to stably express firefly luciferase (Dickson et al., 2007). Luciferase-labeled SJG2 cell line was used to generate the cranial orthotopic tumor model. 


\subsubsection{SJG2 orthotopic tumor xenografts}

8-12 week old CD1 athymic nude mice (Charles River Laboratories, Wilmington, MA) were anesthetized with $100 \mathrm{mg} / \mathrm{kg}$ ketamine and $10 \mathrm{mg} / \mathrm{kg}$ xylazine. With the guidance of a mouse brain atlas (Paxinos and Franklin, 2001), 5x10 SJG2 cells suspended in $5 \mu \mathrm{L}$ Matrigel (Becton Dickinson, Bedford, MA) were injected stereotactically (David Kopf Instruments, Tujunga, CA) into the right caudate putamen. A microdialysis guide cannula (MD-2255, Bioanalytical Systems, West Lafayette, IN) was stereotactically implanted at the same coordinates of the tumor. Tumor growth was monitored by bioluminescence (IVIS-100 imaging system, Xenogen, Alameda, CA (Szentirmai et al., 2006). Briefly, mice were injected intraperitoneally with $120 \mathrm{mg} / \mathrm{kg}$ D-luciferin firefly (potassium salt) (Caliper Lifesciences, Hopkinton, MA) and imaged under isoflurane anesthesia after 5 minutes. All animal studies were carried out in compliance with the Animal Care and Use Committee at St. Jude Children's Research Hospital.

\subsubsection{Magnetic Resonance Imaging (MRI)}

MRI exams were performed as previously described (Elmeliegy et al., 2011a). Briefly, T2 weighted transverse images were generated with turbo spin echo (TSE) protocols for transverse images and turbo gradient protocols for coronal plane using a 7-Tesla Bruker Clinscan (Bruker BioSpin MRI GmbH, Germany) from animals anesthetized using isoflurane (2-3\%). Images were read on a Siemens workstation using Syngo MR B15 software (Siemens, Erlangen, Germany).

\subsubsection{Determination of crenolanib protein binding in mouse plasma}

Crenolanib was added to mouse plasma (Hiltop, Scottdale, PA) to make final concentrations of 250 and $500 \mathrm{ng} / \mathrm{mL}$. Crenolanib-spiked plasma $(200 \mu \mathrm{L})$ was added to each well of a 96-well equilibrium dialysis plate (Harvard Apparatus, MA) and incubated on a rotator at $37^{\circ} \mathrm{C}$. Samples were collected from plasma and PBS buffer chambers at several time points after starting the experiment. Samples were analyzed using LC $\mathrm{MS} / \mathrm{MS}$. Unbound fraction $\left(f_{\mathrm{u}}\right)$ of crenolanib was calculated as follows:

$$
f \mathrm{u}=C_{\mathrm{PBS}} / C_{\text {Plasma }}
$$

where $\mathrm{C}_{\mathrm{PBS}}$ and $\mathrm{C}_{\mathrm{Plasma}}$ are the concentrations of crenolanib in PBS and plasma, respectively.

\subsubsection{Crenolanib and erlotinib steady state plasma pharmacokinetics}

For animals receiving crenolanib or erlotinib as single agents, a $30 \mathrm{mg} / \mathrm{mL}$ solution of either drug in DMSO was loaded in mini-osmotic pumps (Alzet, Model 
2001D; Durect, Palo Alto, CA) and subcutaneously implanted in CD1 nude mice. For animals receiving both agents in combination, a solution containing $30 \mathrm{mg} / \mathrm{mL}$ of each crenolanib and erlotinib, was loaded in the mini-osmotic pumps with subsequent subcutaneous implantation. To determine the time required to achieve steady state, plasma samples were taken by retro-orbital bleeding at 5 time points after drug administration (3, 6, 12, 18, and 24 hours). At least three different animals contributed to each time point. Blood samples were centrifuged at $3000 \mathrm{rpm}$ for 3 minutes. Plasma was immediately separated and stored at $-80^{\circ} \mathrm{C}$ until further analysis using LC MS/MS.

\subsubsection{Cerebral Microdialysis}

Microdialysis experiments were performed in mice implanted with SJG2 cells 28 to 42 days after tumor implantation in tumors whose bioluminescence signal was $\geq 10^{8}$ photons per second. On the day of the experiment, a microdialysis probe (MBR-1-5, Bioanalytical Systems, West Lafayette, IN) was inserted through the cannula and perfused at $0.5 \mu \mathrm{L} / \mathrm{min}$ flow rate with $10 \% \mathrm{HPBCD} / \mathrm{aCSF}$. The probe membrane was allowed to equilibrate for $1 \mathrm{~h}$. Under slight isoflurane anesthesia (2.5\% in oxygen), miniosmotic pump loaded with crenolanib only, erlotinib only, or both, was then subcutaneously implanted. Dialysates were collected using a fraction collector CMA 142 (North Chelmsford, MA) at four different flow rates of $0.5,1,2$, and $4 \mu \mathrm{L} / \mathrm{min}$. Dialysate concentrations were allowed to stabilize for 1 to 2 hours between each change. At least two samples were collected at each flow rate. Dialysates were collected 6-8 hours after pump implantation to ensure achievement of steady state in mouse plasma. Samples were then analyzed using a previously described LC MS/MS method for erlotinib (Elmeliegy et al., 2011b) and the above described LC MS/MS method for crenolanib. Erlotinib and crenolanib concentrations were plotted against flow rate. Extrapolation to a hypothetical ZFR was performed as previously described (Menacherry et al., 1992) using non-linear regression analysis in GraphPad Prism (GraphPad Software, San Diego, CA) using the following formula:

$$
\mathrm{C}_{\text {Dial }}=\mathrm{C}_{0} * \exp (-\mathrm{rA} * \mathrm{X})
$$

where $\mathrm{r}$ (mass transport coefficient) and $\mathrm{A}$ (surface area of the dialysis membrane) are constants, estimated using non-linear regression, $\mathrm{C}_{0}$ represents drug concentrations in the dialyzed tissue, $\mathrm{C}_{\text {Dial }}$ is drug concentration in dialysates, and $\mathrm{X}$ is the flow rate at which the dialysate was obtained at different flow rates.

To ensure achievement of steady state in plasma, at least two plasma samples were obtained from each mouse during the microdialysis experiment. Unbound drug concentrations in plasma were obtained by multiplying total drug concentration by the appropriate unbound fraction $\left(f_{\mathrm{u}}\right)$. The extent of tumor penetration $\left(P_{\text {Tumor }}\right)$ was calculated as a ratio between steady state tumor ECF concentration to-unbound plasma concentration: 


$$
P_{\text {tumor }}=\frac{\text { Tumor } E C F_{\mathrm{ss}}}{\text { Plasma } S S}
$$

where Tumor $E C F_{s s}$ is the $\mathrm{C}_{0}$ obtained from non-linear regression of dialysate concentrations obtained at different flow rates and Plasma SS is the average plasma concentrations obtained from each mouse.

\subsubsection{Statistics}

Data are presented as mean $\pm \mathrm{SD}$. Mann-Whitney test was used for non-paired comparisons of two groups of non-normally distributed data.

\subsection{RESULTS}

\subsubsection{HPBCD improves crenolanib recovery in vitro}

Our previous experience with low probe recovery for erlotinib, a highly lipophilic agent, led us to include the affinity-based trapping agent HPBCD in the perfusate to improve crenolanib probe recovery, as crenolanib is also a highly lipophilic agent. In vitro microdialysis experiments showed that crenolanib had relatively low relative recovery of $12.2 \% \pm 3.1 \%$ at $0.5 \mu \mathrm{L} / \mathrm{min}$ flow rate using aCSF as a perfusate. The addition of $10 \%$ HPBCD in aCSF improved the recovery of crenolanib by 2.4-4.2-fold, depending on the flow rate (Table 4-1). Further, we tested the effect of different flow rates $(0.5,1,2$, and $4 \mu \mathrm{L} / \mathrm{min})$ on relative recovery. Increasing the flow rate yielded lower relative recovery. Even with higher flow rates, crenolanib could be detected in all microdialysis samples. Binding of crenolanib to microdialysis tubing was slightly reduced when HPBCD was added to the perfusate (Table 4-2). Stock concentrations estimated using non-linear regression analysis were within $\pm 15 \%$ accuracy of the starting concentration, indicating that ZFR was a suitable method for estimating probe recovery for crenolanib (Figure 4-1) (Menacherry et al., 1992).

Retrodialysis method involves perfusing the probe with a known concentration of the analyte under the assumption that the diffusion process is quantitatively equal in both directions (i.e. percentage of drug lost from the probe = percentage of drug gained from the medium to the dialysate) (Figure 1-5) (Wang et al., 1993a). Thus, we performed in vitro microdialysis experiments to determine whether this assumption applies when affinity-based trapping agents such as HPBCD are included in the perfusate. Using $10 \%$ $\mathrm{HPBCD} / \mathrm{aCSF}$ as a perfusate, the percentage of crenolanib lost was $3.2 \pm 1.4$ fold lower than that recovered from the stocks [Drug lost $(\%)<<$ Drug gained (\%)]. Thus, the basic assumption of retrodialysis method could not be verified using $10 \% \mathrm{HPBCD} / \mathrm{aCSF}$ perfusate. Since we could not use retrodialysis, we used the ZFR method, which should be applied at steady state conditions. 
Table 4-1. In vitro recovery of crenolanib

\begin{tabular}{cccc}
\hline Flow rate $(\boldsymbol{\mu} \mathbf{L} / \mathbf{m i n})$ & aCSF & $\begin{array}{c}\mathbf{1 0 \%} \text { HPBCD/aCSF } \\
\text { (Relative recovery \%) }\end{array}$ & Fold improvement \\
\hline 0.5 & $12.2 \% \pm 3.1 \%$ & $52.4 \% \pm 9.9 \%$ & 4.2 \\
1 & $9.4 \% \pm 1.5 \%$ & $36.5 \% \pm 22.5 \%$ & 3.8 \\
2 & $8 \% \pm 3.4 \%$ & $19.3 \% \pm 8.6 \%$ & 2.4 \\
4 & $5.1 \% \pm 1.3 \%$ & $12.4 \% \pm 6.8 \%$ & 2.4 \\
\hline
\end{tabular}

Microdialysis probes were inserted in a $30 \mathrm{ng} / \mathrm{mL}$ stock solution of crenolanib and perfused aCSF or $10 \% \mathrm{HPBCD} / \mathrm{aCSF}$. In vitro recovery values (\%) were calculated as $\mathrm{C}_{\text {Dial }} / \mathrm{C}_{\text {stock }} * 100$, where $\mathrm{C}_{\text {Dial }}$ and $\mathrm{C}_{\text {stock }}$ are crenolanib concentration in the dialysates and stocks, respectively. Data are reported as mean $\pm \mathrm{SD}$. Fold improvement was calculated the ratio between the mean recovery value using $10 \% \mathrm{HPBCD} / \mathrm{aCSF}$ and aCSF. 
Table 4-2. Binding of crenolanib to microdialysis tubings

\begin{tabular}{cccc}
\hline Flow rate $(\boldsymbol{\mu L} / \mathrm{min})$ & aCSF & $\begin{array}{c}\mathbf{1 0 \%} \text { HPBCD/aCSF } \\
(\% \text { bound to tubings })\end{array}$ & $\begin{array}{c}\text { Fold reduction in } \\
\text { drug binding }\end{array}$ \\
\hline 1 & $46.1 \% \pm 8.6 \%$ & $23.7 \% \pm 14.1 \%$ & 1.94 \\
\hline
\end{tabular}

Stock solution of crenolanib (30 ng/mL) was prepared in aCSF or $10 \%$ HPBCD and pumped through the microdialysis tubings. Samples are collected and crenolanib concentrations in both the stocks and the collected samples are analyzed. Extent of crenolanib binding to the tubing (\%) was calculated as:

$$
\text { Drug Binding to tubing }(\%)=\frac{\text { Cstock }- \text { Ccollected sample }}{\text { Cstock }} \times 100
$$

where Cstock is crenolanib concentration in the stock pumped in the tubings and Ccollected sample is crenolanib concentration in the sample coming from the other end of the tubings. Data are reported as mean \pm SD of 3 observations at $1 \mu \mathrm{L} / \mathrm{min}$. Drug binding was not significantly different between groups $(P=0.1$, Mann-Whitney test $)$, though there was a trend for reduced binding using $10 \% \mathrm{HPBCD} / \mathrm{aCSF}$. Fold improvement was calculated as the ratio between the mean $\%$ drug bound using $10 \%$ aCSF and 10\% HPBCD. 


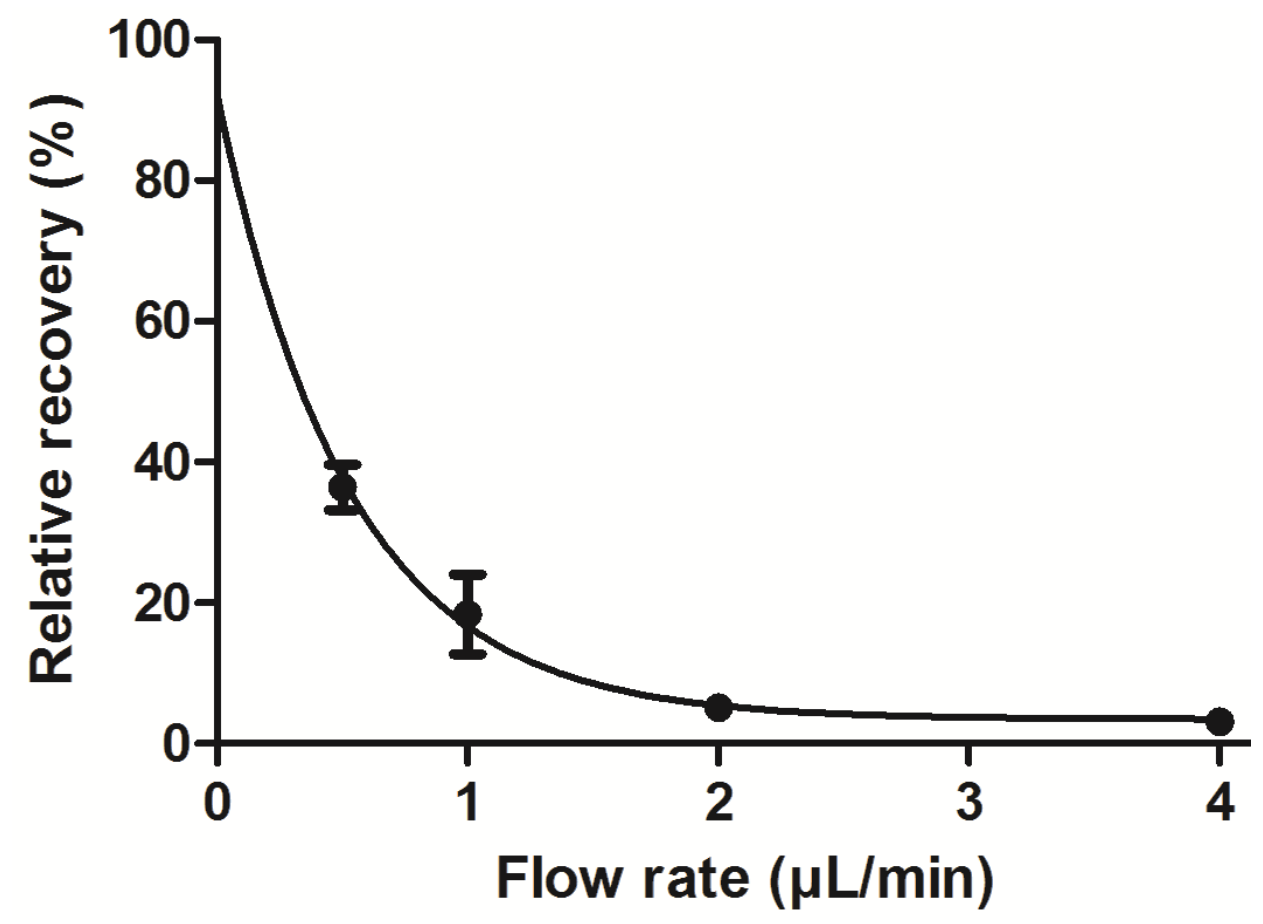

Figure 4-1. Validation of the ZFR method

Crenolanib stocks (30ng/mL) were prepared; microdialysis probes were inserted in stocks and perfused with 10\% HPBCD in aCSF. Dialysates were collected at different flow rates and relative recoveries $(\%)$ were plotted against flow rate. $\mathrm{C}_{\mathrm{ECF}}$ in vitro was estimated using non-linear regression to a hypothetical zero flow rate to be $26.28 \mathrm{ng} / \mathrm{mL}$ (i.e. $87.46 \%$ of the nominal concentration of $30 \mathrm{ng} / \mathrm{mL}$ ). 


\subsubsection{Tumor growth kinetics and morphology}

We studied crenolanib and erlotinib penetration in an orthotopically implanted xenograft model of luciferase-labeled SJG2 glioma cells. The rate of engraftment of SJG2 cells in CD1 nude mice was $85 \%$ (30/35). SJG2 tumors grew as mildly infiltrative tumors invading through the brain parenchyma. These tumors were commonly associated with the development of ventricular edema (Figure 4-2A). SJG2 tumors reached the threshold bioluminescence signal, $10^{8}$ photons per second, within 4-6 weeks postimplantation (Figure 4-2B), and neurologic signs were observed within 5 weeks.

\subsubsection{Crenolanib and erlotinib steady state plasma pharmacokinetics}

Determining the time required to achieve steady state in vivo is important in designing the sampling strategy when using the ZFR method to calculate in vivo probe recovery. We conducted a plasma pharmacokinetic study of crenolanib only, erlotinib only, and crenolanib/erlotinib combination to determine the time required to reach steady state drug concentrations in mouse plasma. Mini-osmotic pumps loaded with crenolanib, erlotinib, or both agents in DMSO were subcutaneously implanted in CD1 nude mice at a target dosage of $200 \mathrm{mg} / \mathrm{kg} /$ day. The drug release rate from these pumps is $240 \mu \mathrm{g} / \mathrm{hr}$ for at least 24 hours. This dosage was well tolerated by the mice as visually observed by normal respiratory rate, ability of the animal to defecate/urinate, regular access to food and water, and absence of any neurological signs. Steady state levels of both crenolanib and erlotinib were achieved within 6 hours post-implantation and were maintained for at least 24 hours (Figure 4-3), as determined by comparing drug concentrations at 6 hours to that at 3 and 24 hours. Plasma levels at 6 hours were significantly higher than at 3 hours (Mann-Whitney test, $p<0.05$ ), but similar to that observed at 24 hours (Mann-Whitney test, $p>0.2$; Figure 4-3).

\subsubsection{Crenolanib protein binding in mouse plasma}

Using equilibrium dialysis, we assessed the extent of crenolanib protein binding in mouse plasma. The concentrations used were similar to those expected after administering crenolanib to mice at $200 \mathrm{mg} / \mathrm{kg}$ using mini-osmotic pumps (i.e., 250 and $500 \mathrm{ng} / \mathrm{mL}$ ). The time required to reach equilibrium was 18 hours. Crenolanib was found to be highly protein bound in mouse plasma. Unbound fraction $\left(f_{\mathrm{u}}\right)$ of crenolanib was $5.9 \% \pm 0.5 \%$ and $6.1 \% \pm 0.7 \%$ for the 250 and $500 \mathrm{ng} / \mathrm{mL}$ concentrations, respectively (Figure 4-4).

\subsubsection{Crenolanib penetration in SJG2 xenografts}

We used cerebral microdialysis to sample crenolanib penetration in an orthotopic mouse model of glioblastoma (i.e. SJG2). Crenolanib was administered as a single agent subcutaneously using mini-osmotic pumps (target dosage $200 \mathrm{mg} / \mathrm{kg}$ ). Using 10\% 


\section{A.}
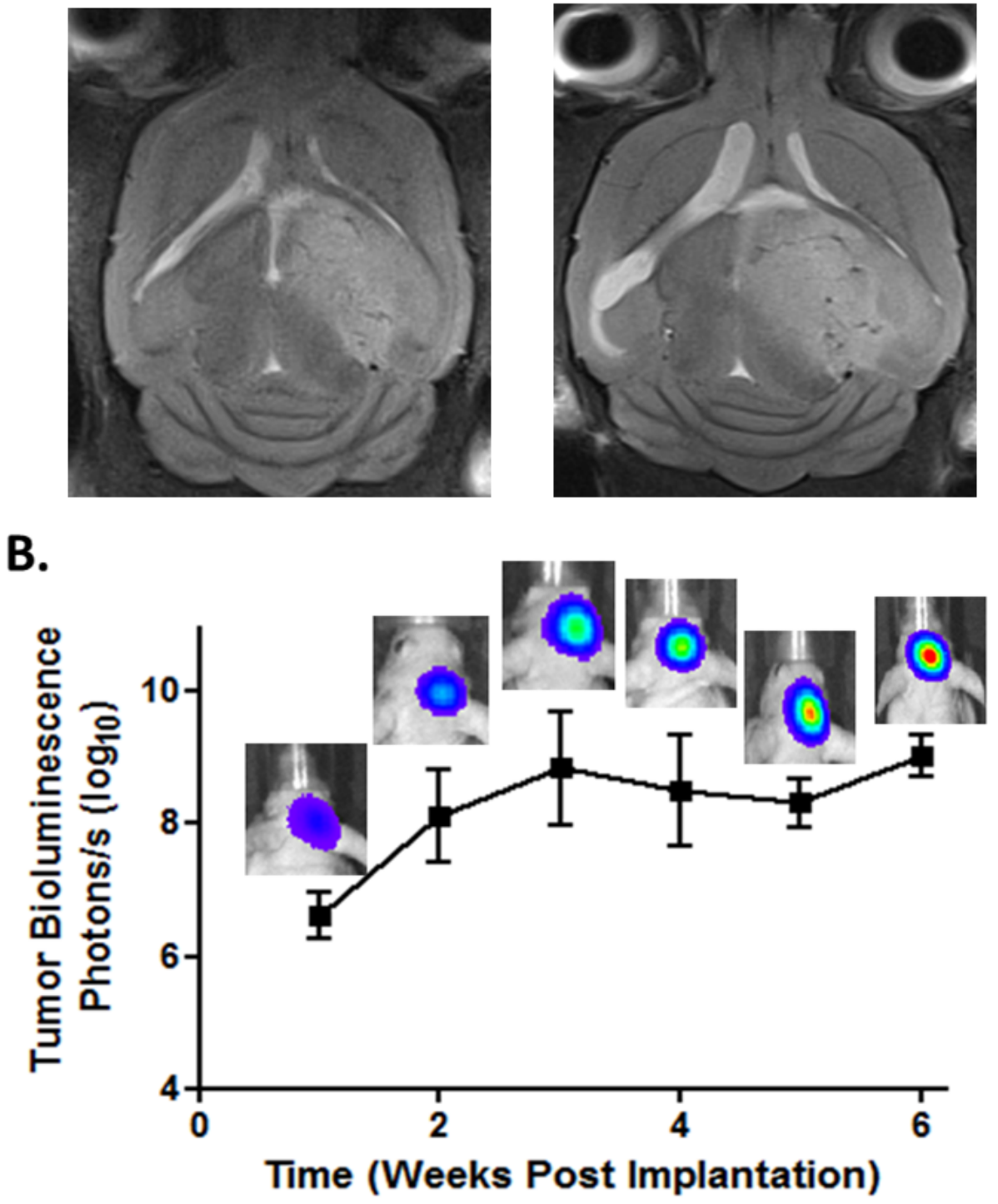

Figure 4-2. Growth characteristics of SJG2 tumors

A. T2-weighted MRI of two representative SJG2 tumors displaying invasive growth pattern through the normal brain parenchyma with ventricular edema.

B. SJG2 tumor bioluminescence signal versus time. Data are presented as mean and SD of 4-10 values per time point. Representative bioluminescence pictures are shown. 


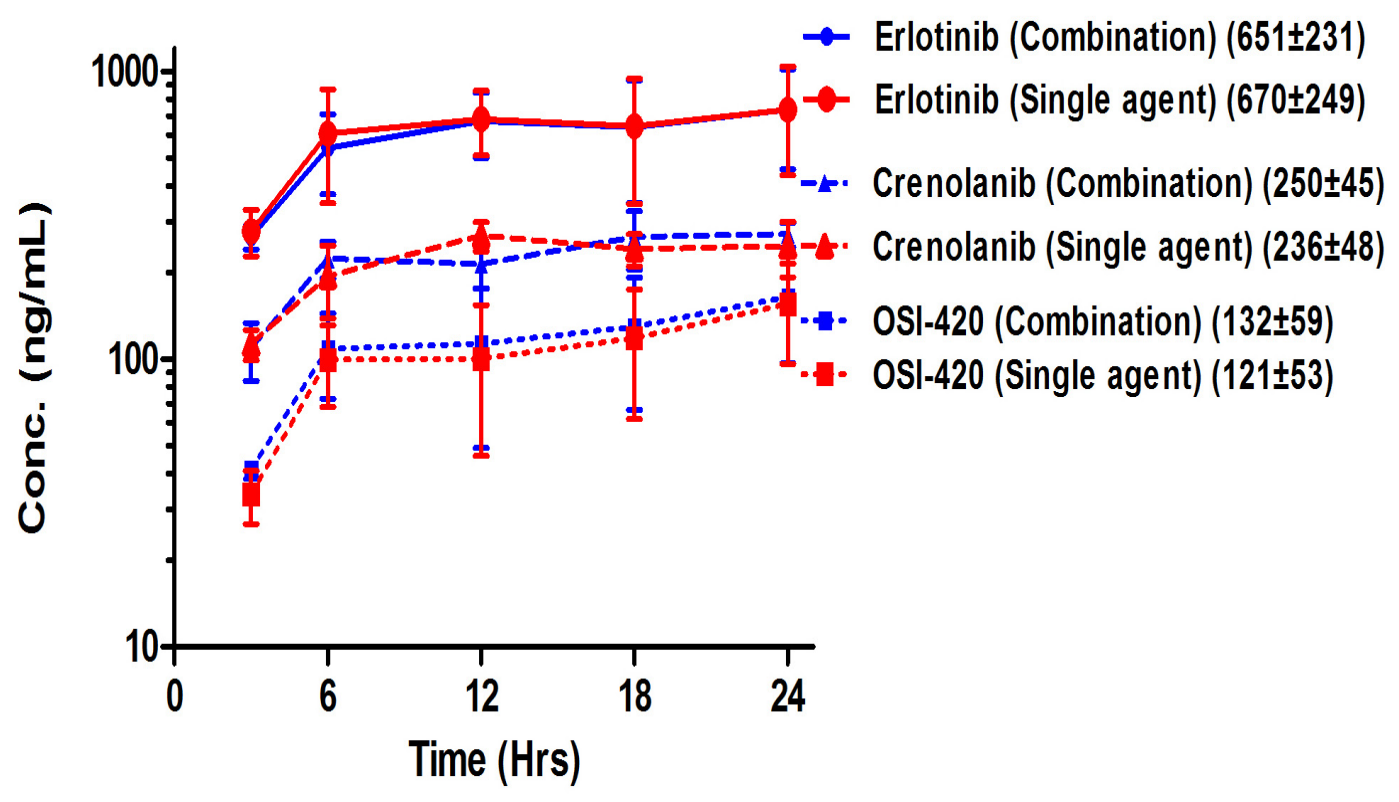

Figure 4-3. Concentration-time profile for crenolanib, erlotinib, and OSI-420 after implantation of mini-osmotic pumps in CD1 nude mice

Concentrations obtained after single agent administration of crenolanib or erlotinib (red lines) were similar to that obtained after administering both agents in combination (blue lines) $(\mathrm{p}>0.05)$. Steady state levels were achieved 6 hours post-implantation. Values for steady state concentrations are reported on figure in $\mathrm{ng} / \mathrm{mL}$ as mean $\pm \mathrm{SD}$ of values obtained at $6,12,18$, and 24 hours. 


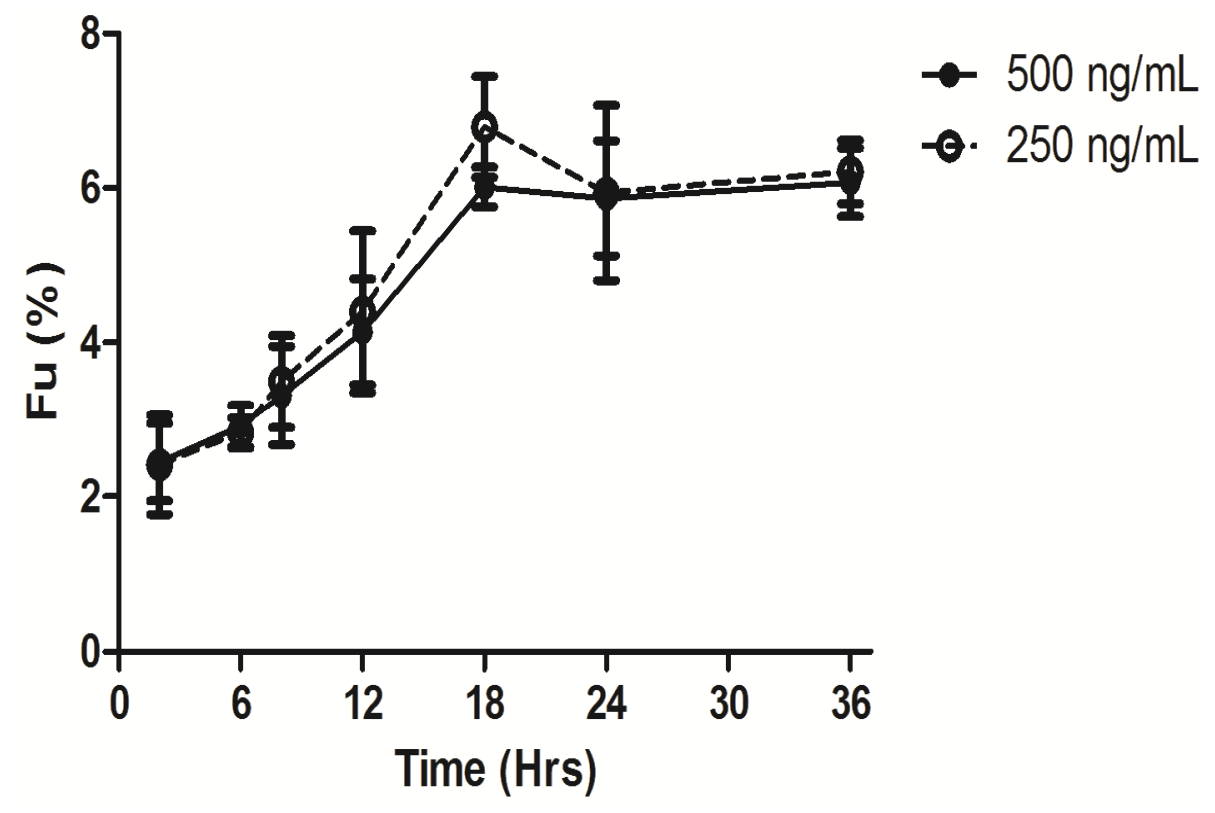

Figure 4-4. Crenolanib is highly bound to plasma proteins

Crenolanib plasma protein binding assessed using equilibrium dialysis at two concentrations $(250$ and $500 \mathrm{ng} / \mathrm{mL})$. Unbound fraction $\left(f_{\mathrm{u}}\right)$ was calculated as mean values from 18, 24, and 36 hours. 
$\mathrm{HPBCD} / \mathrm{aCSF}$ as perfusate and sensitive analytical methods, we were able to detect crenolanib in dialysates. The mean $\pm \mathrm{SD}$ for crenolanib penetration in SJG2 tumors was $P_{\text {Tumor }}=0.19 \pm 0.03, \mathrm{n}=4$ (Figure 4-5).

A second cohort of mice received crenolanib and erlotinib in combination (target dosage of $200 \mathrm{mg} / \mathrm{kg}$ of each). Collected dialysates were split for analysis of crenolanib and erlotinib/OSI-420. Crenolanib penetration in mice receiving crenolanib and erlotinib $\left(P_{\text {Tumor }}=0.13 \pm 0.05, \mathrm{n}=6\right)$ was similar to that in mice receiving crenolanib alone (Mann-Whitney, $P=0.07$; Figure 4-5).

\subsubsection{Erlotinib penetration in SJG2 xenografts}

Erlotinib was administered as a single agent using mini-osmotic pumps (target dosage $200 \mathrm{mg} / \mathrm{kg}$ ). The mean $\pm \mathrm{SD}$ of erlotinib penetration in SJG2 xenografts was $P_{\text {Tumor }}=0.25 \pm 0.03 \mathrm{n}=4$ (Figure 4-5). For the combination group, mice received 200 $\mathrm{mg} / \mathrm{kg}$ of each crenolanib and erlotinib. In this group, erlotinib penetration $\left(P_{\text {Tumor }}=0.26\right.$ \pm 0.06 , mean $\pm \mathrm{SD}, \mathrm{n}=6$ ) was similar to that in mice receiving erlotinib alone (Mann-Whitney, $P=0.47$ ). OSI-420, the major erlotinib metabolite, was not detected in the dialysates in any of the tested groups (single agent or combination) (Figure 4-5).

\subsection{DISCUSSION}

Previous studies have reported irreproducible dialysis of lipophilic drugs (Lindberger et al., 2002; Loos et al., 2007; Wang et al., 2008). In addition, previous studies have only evaluated a single anti-cancer agent, an approach that has limited relevance in light of the prevalent use of combinations of two or more anti-cancer drugs in therapy. Here, we added the affinity-based trapping agent HPBCD to the perfusate to increase microdialysis probe recovery of two lipophilic anti-cancer drugs, crenolanib and erlotinib. We then used this method to determine crenolanib and erlotinib tumor penetration as single agents or when administered in combination.

A major limitation of cerebral microdialysis is associated with the poor recovery of lipophilic compounds due to the tendency of these compounds to stick to the sampling probe and tubing components, poor solubility of these compounds in the hydrophilic perfusate, and their high protein binding affinity which limits the unbound drug in the sampling space (Benfeldt and Groth, 1998; Benveniste and Huttemeier, 1990; Carneheim and Stahle, 1991; de Lange et al., 2000; Lindberger et al., 2002; Oravcova et al., 1996). Several attempts to improve recovery of lipophilic compounds involved replacing the commonly used hydrophilic perfusates with hydrophobic solutions such as $2.25 \%$ glycerol in water or $20 \%$ soybean oil/ egg phospholipid (Intralipid®) which allow the lipophilic drug to accumulate in the perfusate (Carneheim and Stahle, 1991; Kurosaki et al., 1998). Other attempts involved adding BSA in the perfusate where the drug can bind to BSA rather than the sampling device (Carneheim and Stahle, 1991). While these approaches are often successful in improving the recovery of hydrophobic compounds, 


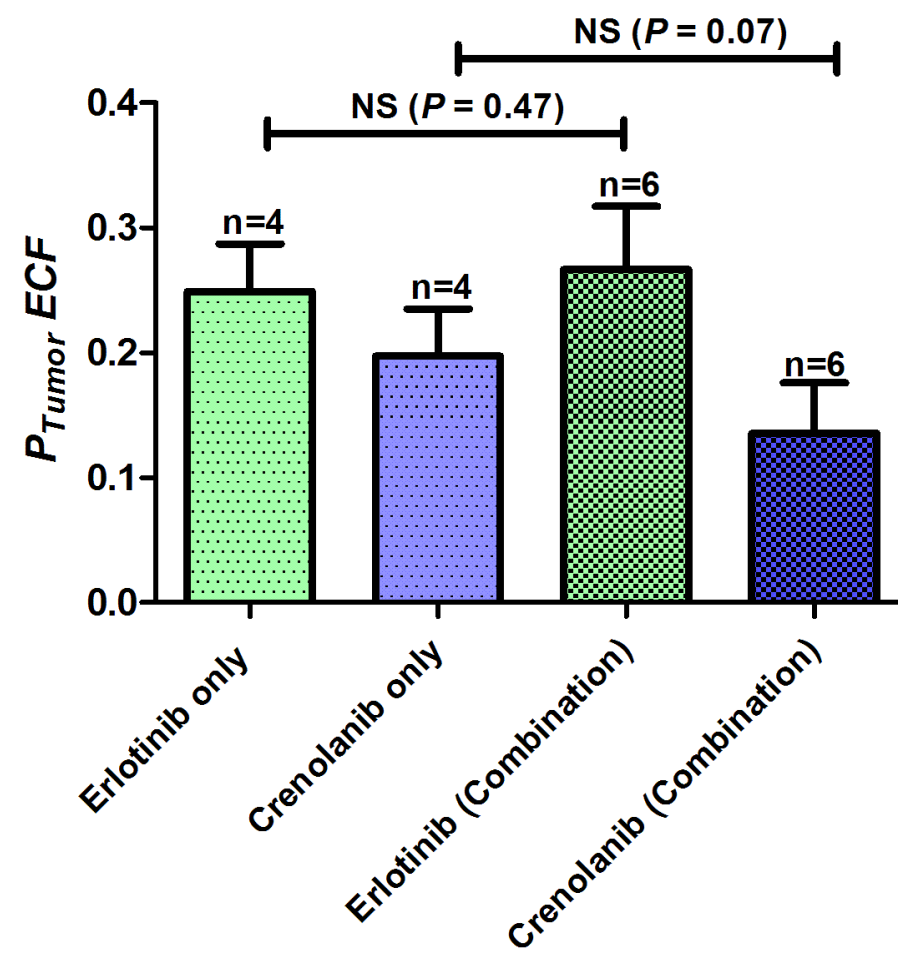

Figure 4-5. Crenolanib and erlotinib penetration in SJG2 xenografts

Drugs were administered via mini-osmotic pumps. Tissue ECF was sampled via cerebral microdialysis using $10 \% \mathrm{HPBCD}$ /aCSF perfusate and using the ZFR recovery approach. Mouse plasma samples were obtained via retro-orbital bleeding and corrected for protein binding. Extent of tumor penetration $\left(P_{\text {Tumor }}\right)$ was expressed as the ratio between tissue concentration at hypothetical ZFR and unbound drug in mouse plasma. Tumor penetration of unbound crenolanib and erlotinib after single agent or combination administration of $200 \mathrm{mg} / \mathrm{kg} /$ day crenolanib, erlotinib, or both, via mini-osmotic pumps in SJG2 mice. No statistical difference was observed in drug penetration between single agent and combination groups, $P>0.05$. Statistical analysis was performed using Mann Whitney test. 
they add technical challenges during the experiment (i.e., flow resistance and tube blockage, as well as analytical challenges during sample cleanup for LC-MS/MS assays) (Elmeliegy et al., 2011b; Jensen et al., 2007; Sun and Stenken, 2003).

Another approach is the use of cyclodextrins in the perfusate (Khramov and Stenken, 1999a; b). Cyclodextrins form complexes with lipophilic molecules by capturing them in a non-polar central cavity (Loftsson and Brewster, 1996). Upon including cyclodextrins in the perfusate, they form an inclusion complex with the analyte on the dialysate side of the membrane reducing the effective concentration of the analyte on the dialysate side. This results in a larger concentration gradient that facilitates more diffusion from the target tissue to the dialysate which leads to higher recovery (Khramov and Stenken, 1999a). Advantages of using HPBCD include its high safety profile (Gould and Scott, 2005) and that it can be directly injected on the HPLC minimizing sample processing prior to analysis (Italia et al., 1990; Maguire, 1987).

While including HPBCD in the perfusate proved technically indispensable in our experiments, it also added some experimental challenges in estimating probe recovery. During recovery estimation, HPBCD can form inclusion complexes with the drug preventing its passage through the probe and thus all recovery estimation methods that involve perfusing the probe with drug solution, e.g. retrodialysis, cannot be used to estimate probe recovery should HPBCD be included in the perfusate. This mandates the use of other recovery methods that do not involve drug perfusion through the probe. These methods usually require constant drug concentrations while determining the recovery, such as ZFR method (Elmeliegy et al., 2011b).

In vivo microdialysis experiments showed that crenolanib and erlotinib could be simultaneously dialyzed and detected in the dialysates. However, we could not detect OSI-420 in most of our microdialysis samples. We have previously reported that OSI-420 exhibits low penetration profile to mice brains ( $7 \%$ ) (Elmeliegy et al., 2011b). Since average steady state concentrations of OSI-420 in mice plasma was $132 \mathrm{ng} / \mathrm{mL}$ (i.e. $\sim 6$ $\mathrm{ng} / \mathrm{mL}$ of unbound OSI-420 in plasma), therefore OSI-420 concentrations in the brain and that in the dialysates are expected to be below the LLOQ of our LC/MS-MS assay $(0.5 \mathrm{ng} / \mathrm{mL})$.

We decided to use the steady state approach for dosing crenolanib and erlotinib for two reasons. First, the ZFR recovery method, employed in these experiments due to the presence of HBCD requires steady state drug levels at the target tissue. Second, crenolanib has a shorter half-life in mice (t1/2 of crenolanib in CD1 nude mice $=0.53 \pm 0.11$ hours, Dr. Clinton Stewart, unpublished data) than in humans ( $\mathrm{t} 1 / 2$ of crenolanib $=14 \pm 4.18$ hours) (Lewis et al., 2009). Clinical trials of either crenolanib or erlotinib involve long-term chronic oral daily administration, which will result in steady state drug concentrations in patient plasma (Broniscer et al., 2007c; Lewis et al., 2009). Thus, studying CNS penetration of these drugs under steady state conditions in mice could provide better recapitulation of the clinical situation. 
While the penetration of crenolanib to SJG2 tumors was similar between the single-agent and combination administrations, overall crenolanib tumor penetration was relatively low $\left(P_{\text {Tumor }}=0.19\right.$ and 0.13$)$, especially when compared to penetration of other compounds in mouse models of glioma (Agarwal et al., 2011a; Carcaboso et al., 2010; Vogelbaum et al., 2011; Wang et al., 2010; Zhou and Gallo, 2009). One possible explanation is the relatively high molecular weight of crenolanib, 601.72 Daltons, which may limit its passive diffusion through the BBB (Kniesel and Wolburg, 2000; Mahar Doan et al., 2002). Another potential explanation could be active crenolanib transport from brain to blood via efflux transporters at the endothelial cells of the BBB (Terasaki and Ohtsuki, 2005). Finally, an intact BBB in SJG2 xenograft model might also limit crenolanib penetration.

Despite the relatively low penetration of crenolanib in $\mathrm{SJG} 2$ tumors (mean $\pm \mathrm{SD}$ of crenolanib concentration in SJG2 tumors ECF was $2.55 \pm 0.81 \mathrm{ng} / \mathrm{mL} \mathrm{n}=4$ ), PDGFR $\alpha$ phosphorylation was inhibited in $66 \%$ (2/3) of SJG2 tumors treated with crenolanibloaded mini-osmotic pumps for 24 hours (data not shown). While $2.55 \mathrm{ng} / \mathrm{mL}$ of crenolanib might be lower than crenolanib IC50 for PDGFR $\alpha$ determined using western blot experiments $(8.1 \mathrm{ng} / \mathrm{mL}$ ) (data not shown), our experimental methodology could not determine the concentration of free drug in the intracellular compartment. Given its high lipophilic nature, crenolanib might accumulate in the intracellular compartment in concentrations sufficient to inhibit PDGFR $\alpha$ activation.

In conclusion, cerebral microdialysis can be used to simultaneously sample lipophilic anti-cancer agents. Inclusion of HPBCD increased probe recovery of crenolanib and erlotinib. Sensitive analytical techniques are needed for successful implementation of simultaneous sampling via cerebral microdialysis. This method will increase our ability to conduct CNS penetration studies for multiple anti-cancer drugs administered in combination. 


\section{CHAPTER 5. SUMMARY, DISCUSSION AND FUTURE DIRECTIONS}

Primary CNS tumors, particularly malignant gliomas, represent a major therapeutic challenge to basic scientists and neuro-oncologists. Despite decades of research, hundreds of clinical trials, and aggressive treatment strategies, glioma patients still suffer from poor prognosis, poor quality of life, and low overall survival (Broniscer and Gajjar, 2004; Furnari et al., 2007; Wen and Kesari, 2008). Perhaps one of the most important reasons for this poor prognosis is the intrinsic resistance of malignant glioma achieved by effective DNA repair machinery (Frosina, 2009), deletion mutations in several tumor suppressor pathways such as P53 and RB pathways (Paugh et al., 2010b; TCGA, 2008), and simultaneous activation of multiple RTKs (Stommel et al., 2007; TCGA, 2008). These genetic abnormalities maintain glioma cell survival, proliferation, and stimulate its invasive growth pattern through the brain.

Another reason for the clinically challenging nature of malignant glioma is the restricted delivery of anti-cancer agents to the active site in the brain, which is protected by several barrier systems such as the BBB and the BCSFB. The overall objective of this project was to better understand the CNS penetration of molecularly targeted agents currently in clinical trials for treatment of malignant glioma using mouse models of glioma.

While microdialysis offers several technical advantages to understanding CNS drug penetration, it is not without limitations. One challenge to the use of microdialysis is the low probe recovery of lipophilic compounds. The low recovery of these compounds can be attributed to the tendency of these compounds to bind to the sampling components, their poor solubility in hydrophilic perfusates, their high protein binding affinity (which limits the unbound drug in the sampling space), and their tendency to accumulate in the intracellular compartment, which reduces the sampling pool in tumor ECF (Lindberger et al., 2002; Loos et al., 2007; Wang et al., 2008). This challenge is particularly significant because many of the compounds under development for treatment of brain tumors tend to exhibit lipophilic properties.

To address this issue we chose to include an affinity-based trapping agent (HPBCD) in the perfusate to increase probe recovery of erlotinib and crenolanib, two highly lipophilic agents. This resulted in an eight- and four-fold increase in probe recovery for erlotinib and crenolanib, respectively. This approach has been successfully applied to enhance probe recovery of several lipophilic agents such as gefitinib, lapatanib, BEZ-235, and OSI-906 (data not shown). However, even with HPBCD in the perfusate, we could not detect sirolimus, an mTOR inhibitor, in dialysates obtained from U87-MG xenografts mouse models receiving $50 \mathrm{mg} / \mathrm{kg}$ sirolimus by oral gavage (data not shown). This may be explained by the large molecular weight of sirolimus (914 Daltons), inability of HPBCD to form inclusion complex with sirolimus, or possibly due to low sirolimus penetration in these tumors. 
While including HPBCD in the perfusate proved technically indispensable in our experiments, it also added experimental challenges. During recovery estimation, HPBCD forms inclusion complexes with the drug and thus recovery estimation methods that involve perfusing the probe with drug solution, such as retrodialysis, could not be used to estimate probe recovery. This mandates the use of other recovery methods (e.g., ZFR), which require a constant drug concentration in the target tissue (Elmeliegy et al., 2011b).

Using HPBCD in the perfusate and the ZFR method for in vivo recovery estimation, we studied erlotinib and OSI-420 penetration in control and transporterdeficient mice receiving erlotinib by oral gavage. We showed that Bcrp1 was the main efflux transporter restricting erlotinib and OSI-420 penetration in mouse brain.

Intracellular accumulation studies confirmed the role of BCRP in erlotinib and OSI-420 transport. Furthermore, we showed that OSI-420 is a P-gp substrate while erlotinib is not. OSI-420 is formed by removing one methyl group of erlotinib by CYP3A4. The different transport profiles of erlotinib and OSI-420 by P-gp suggests that even this slight structural difference between erlotinib and OSI-420 may affect the affinity of efflux transporters to their substrates (Elmeliegy et al., 2011b).

BCRP and P-gp were shown to interact with several tyrosine kinase inhibitors. Both transporters are expressed on the endothelial cells forming the BBB, the epithelial cells of the choroid plexus forming the BCSFB, and on the glioma cell membrane. Thus, they can influence the disposition of their substrates in different CNS compartments (i.e., vCSF, brain ECF, or inside tumor cells). Further, these transporters are highly expressed in the small intestine and the colon and thus may reduce the oral bioavailability of their substrates (Lemos et al., 2008).

While the contribution of efflux transporters to CNS disposition of anti-cancer drugs has been extensively studied, the role of solute carrier transporters in CNS and tumor cell uptake of anti-cancer agents remains unclear. We studied the intracellular accumulation of erlotinib and OSI-420 using an array of HEK293 cell lines that over express uptake transporters. Our data show that erlotinib and OSI-420 are substrates for OAT3 and OCT2. We are currently planning studies to determine the contribution of mouse Oct2 in erlotinib brain disposition using $O c t 2^{-/-}$murine models (Jonker et al., 2003). Lower erlotinib accumulation in $O c t 2^{--}$mice would confirm the role of Oct2 in erlotinib transport and indicate the potential importance of this transporter. These studies can also provide further explanation for the high variability observed in erlotinib pharmacokinetics in patients. Immunohistochemical analysis of various uptake and efflux transporters will be conducted on mouse brain sections from wild-type and $O c t 2^{-/}$mice to identify any compensatory changes in transporter expression in Oct2 deficient mice.

We then sought to characterize erlotinib and crenolanib disposition in malignant glioma using cerebral microdialysis. We decided to use a transgenic mouse model of malignant glioma (conditional TKO) that highly recapitulates several features of the human disease including tumor histology and genetic profiles. However, one limitation against using these models in CNS penetration studies using cerebral microdialysis is the inability to identify the bregma point on MRI. Thus, we identified a new reference point 
that relates the MRI scans to the mouse skull. This allowed accurate implantation of the microdialysis guide cannula using MRI-derived coordinates.

Using this model, cerebral microdialysis studies to assess anti-cancer drug penetration in transgenic models where tumors arise spontaneously can be performed. This method can also be used to target catheters implanted stereotactically for local delivery of anti-cancer agents within brain tumors, a technique known as convectionenhanced delivery. Convection-enhanced delivery involves insertion of a catheter directly in the tumor where drugs are then locally delivered through the implanted catheter under continuous pressure. This technique allows the delivery of high intratumoral drug concentrations while reducing systemic side effects. However, accurate catheter placement in the tumor is thought to be crucial for successful delivery of the anti-cancer agent. Inadequate placement of the catheter was shown to cause fluid accumulation in the subarachnoid space, which can be locally toxic. This is due to the high pressure required for delivery of the drug in the tumor (Valles et al., 2009). Given the high success rate of our method in identifying accurate stereotactic coordinates, our method can be applied to accurately target catheters used in convection-enhanced delivery of anti-cancer agents in novel preclinical models.

The use of appropriate preclinical models that reflect the genetic and histological properties of the disease allows for better preclinical testing of novel anti-cancer agents. Both spontaneous and xenograft models provide a set of advantages and disadvantages to the research question. Spontaneous mouse models reproduce the microenvironment of high-grade glial tumors better than xenograft models (Becher and Holland, 2006; Chow and Baker, 2012; Kucherlapati, 2012). However, some concerns exist regarding the practical application of these models in pharmacokinetic investigations given the long latency for tumor development and the need for expensive imaging modalities to monitor tumor growth. Furthermore, tumors that arise spontaneously display greater heterogeneity compared to xenograft models. Indeed, we observed greater variability in tumor penetration and contrast enhancement in the conditional TKO model compared to SJG2 xenografts (data not shown). This can be explained by the heterogeneous tumor growth pattern, location, and histological representation of the tumor in conditional TKO model while SJG2 tumors displayed more consistent growth pattern.

We observed higher crenolanib penetration in the conditional TKO compared to that in SJG2 xenografts (data not shown). This may be explained by the contribution of uptake transporters to crenolanib transport. Additional studies are warranted to investigate the role of these transporters in crenolanib CNS disposition. Studies investigating the role of solute carrier transporters in crenolanib CNS penetration should study crenolanib intracellular accumulation in cell lines that overexpress uptake transporters. Finally, the role of a particular transporter identified in the initial screen should be further confirmed in mouse models deficient of that transporter. Identifying efflux and uptake transporters responsible for crenolanib transport may provide further explanation for the different crenolanib penetration profiles observed in transgenic and xenografts models. The vastly different crenolanib penetration profiles in conditional TKO and SJG2 xenografts indicates the importance of using more than one tumor model 
in drug penetration studies. Different transporters expression and vascular properties among different models can drastically change drug penetration, and thus the use of a single model may be misleading.

To achieve the required anti-tumor effect, tumor cells should be exposed to effective concentrations of the anti-cancer agent for a sufficient duration. It is important to emphasize that only the protein-unbound form at the active site is pharmacologically available to interact with its target and exert a pharmacological effect. Anti-cancer drug disposition within tumor compartments (vascular, extracellular, and intracellular) is controlled by intricate properties of each compartment (Figure 5-1). First, only unbound drug in plasma can diffuse across the BBB to accumulate within the tumor ECF. This process is restricted by tight junctions between endothelial cells of the $\mathrm{BBB}$ and an array of efflux and uptake transporters expressed on these cells. Unbound drug in tumor ECF can then accumulate inside tumor cells. However, intracellular drug accumulation can be influenced by efflux and uptake transporters expressed on glioma cell membranes (Carcaboso et al., 2010). Finally, non-specific binding of the drug in the intracellular compartment will further reduce the drug available to interact with its intracellular target (Figure 5-1) (Baselga, 2006; Hammarlund-Udenaes, 2010; Hammarlund-Udenaes et al., 2008; Reichel, 2009).

Unfortunately, methods for direct determination of unbound intracellular drug concentration are not available. However, future experiments should rely on combining several approaches to estimate unbound intracellular drug concentrations. HammarlundUdenaes and colleagues elegantly proposed an equation that can be used to estimate unbound intracellular drug concentration in brain parenchyma (Hammarlund-Udenaes et al., 2008). This equation can be possibly applied to estimate unbound intracellular drug concentration inside tumor cells:

$$
C u, \text { cell }=\frac{\text { Atumor }(V u, \text { tumor }- \text { Vtumor } E C F)}{\text { Vcell } X \text { Vu, cell } X \text { Vu,tumor }}
$$

where $\mathrm{Cu}$, cell is the unbound drug concentration in the intracellular compartment, Atumor is the total drug in tumor, Vu,tumor is the unbound drug volume of distribution in tumor (See text for explanation). Vtumor ECF and Vcell are the volumes of tumor ECF and tumor cells, respectively, and can be substituted by physiological values of the brain tissue and the astrocyte, respectively. $\mathrm{Vu}$, cell is the volume of distribution of unbound drug in tumor cell.

$\mathrm{Vu}$, tumor describes the relation between Atumor and the unbound drug concentration in tumor ECF regardless of tumor to plasma concentration ratio. $\mathrm{Vu}$, tumor can be calculated using the following equation:

$$
\text { Vu,tumor }=\frac{\text { Atumor }- \text { Avascular }}{C u, E C F}
$$




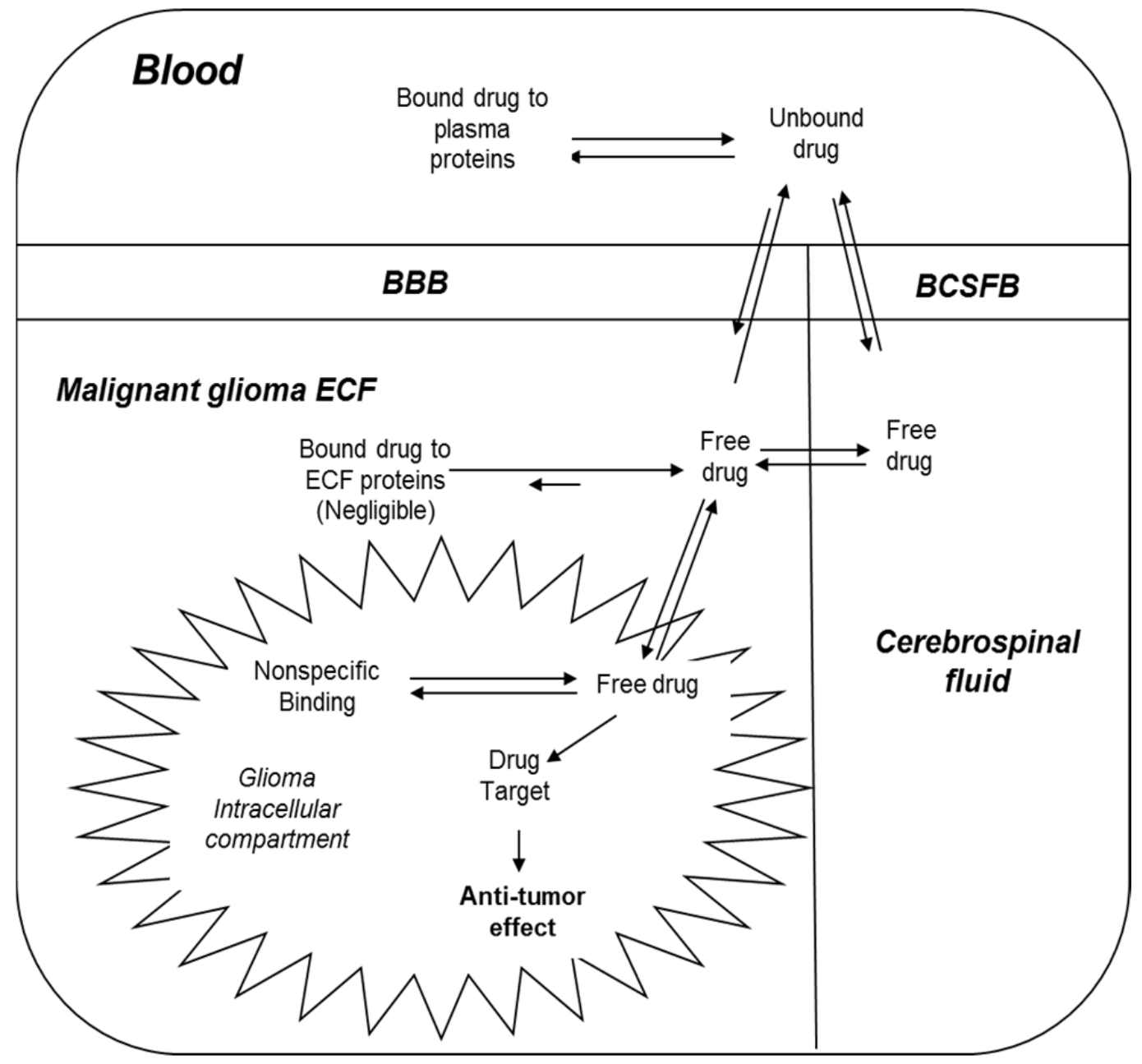

Figure 5-1. Pharmacokinetic compartments of the CNS 
where Avascular is the amount of drug in tumor blood vessels which can be estimated as previously described (Carcaboso et al., 2010) and $\mathrm{Cu}, \mathrm{ECF}$ is the unbound drug concentration in tumor ECF obtained from microdialysis experiments.

Atumor can be determined by tumor homogenization. We have previously used the term $\mathrm{Vu}$, tumor to account for the shift in drug accumulation from the ECF to the intracellular compartment upon inhibiting efflux transporters (Carcaboso et al., 2010). We have previously combined microdialysis and tumor homogenization methods using steady state approach to determine $\mathrm{Vu}$, tumor. Low $\mathrm{Vu}$, tumor value indicates that the drug is more confined to the ECF compartment (i.e. high $\mathrm{Cu}, \mathrm{ECF}$ ) with less intracellular distribution while higher $\mathrm{Vu}$, tumor value suggests that the drug exhibit more intracellular distribution (Carcaboso et al., 2010). However, $\mathrm{Vu}$, tumor does not account for drug intracellular binding and thus cannot provide an estimate for the unbound drug concentration in tumor cells. Homogenate binding experiments can be used to determine the non-specific binding of the drug to the tumor. Determining intracellular binding will allow estimation of $\mathrm{Vu}$,cell. Thus, future experiments should combine different sampling approaches (microdialysis, tumor homogenization, and homogenate binding) to estimate the unbound drug concentration in the intracellular compartment $(\mathrm{Cu}$, cell).

Several factors should be considered upon determining whether tumor penetration of an anti-cancer agent in preclinical models is "adequate" for testing in patients with malignant glioma. First, we should determine the unbound plasma systemic exposure that can be achieved and tolerated in patients enrolled in phase I trials. Second, we should try to recapitulate this exposure in preclinical models to determine whether the unbound drug concentrations achieved in the tumor at this exposure are effective to exert a pharmacological effect.

Upon conducting these studies, the extent of tumor penetration should not be regarded as the only determinant of our decision on whether a certain agent is a good candidate for clinical testing. For example, a drug with high potency against its target can be effective despite its low tumor penetration. This is supported by our data that show $66 \%$ inhibition of PDGFR $\alpha$ in SJG2 xenografts by crenolanib despite its relatively low tumor penetration in SJG2 xenografts.

Furthermore, in vitro and preclinical testing of potential agents should be conducted at exposures similar to that achievable and tolerated in patients. Exerting a pharmacological effect only at exposures that are 10 fold higher than that clinically tolerated suggests a "no-go" decision for testing this drug in patients. In our experiments, steady state concentrations in mouse plasma were $\sim$ two-fold lower compared to concentrations achieved in patients (Broniscer et al., 2009; Lewis et al., 2009). It is important to note that we could not increase the dose administered in our studies as we were limited by drug solubility in DMSO that was loaded in the mini-osmotic pumps.

Despite PDGFR $\alpha$ inhibition by crenolanib, downstream signaling events (e.g., AKT phosphorylation was not affected) (data not shown). This agrees well with previous reports showing redundant signaling of multiple RTKs, such as PDGFR and EGFR, to 
maintain active PI3K signaling in malignant glioma (Stommel et al., 2007). These studies indicate the need for combination regimens to inhibit PI3K pathway and alter glioma survival. Thus, we designed studies in which we combined PDGFR and EGFR inhibitors in our SJG2 xenografts and studied their tumor penetration using cerebral microdialysis. Using HPBCD in our perfusate, ZFR method to estimate in vivo probe recovery, and sensitive analytical techniques, we showed, for the first time, the feasibility of simultaneous sampling of multiple anti-cancer agents via cerebral microdialysis. Future studies should evaluate the effects of this combination on downstream Akt signaling at exposures achievable in the tumors. Our method will enable better understanding of CNS penetration of several drug combinations. Using our method, tumor penetration of several combinations targeting pathways known to maintain glioma survival should be evaluated. Such combinations may include agents that target multiple components of the same pathway (e.g., PDGFR, Akt, and mTOR inhibitors) or those targeting several pathways (e.g., PDGFR and MEK inhibitors).

In conclusion, we developed new techniques to improve microdialysis probe recovery of erlotinib and crenolanib. We identified efflux and uptake transporters that regulate erlotinib CNS disposition. We developed an MRI-guided method to implant microdialysis cannula in a spontaneous glioma murine model. We identified suitable microdialysis conditions used to sample multiple anti-cancer agents in tumor ECF of mouse models of glioma. 


\section{LIST OF REFERENCES}

Agarwal S, Mittapalli RK, Cen L, Carlson BL, Elmquist WF and Sarkaria JN (2011a) Influence of Bevacizumab on the Targeted Delivery of Tyrosine Kinase Inhibitors and Temozolomide in Primary Gbm Xenograft Models. Neuro-Oncology 13:113114.

Agarwal S, Sane R, Gallardo JL, Ohlfest JR and Elmquist WF (2010) Distribution of Gefitinib to the Brain is Limited by P-glycoprotein (ABCB1) and Breast Cancer Resistance Protein (ABCG2) Mediated Active Efflux. J Pharmacol Exp Ther. 334:147-155.

Agarwal S, Sane R, Oberoi R, Ohlfest JR and Elmquist WF (2011b) Delivery of molecularly targeted therapy to malignant glioma, a disease of the whole brain. Expert reviews in molecular medicine 13:17-43

Agarwal S, Sane R, Ohlfest JR and Elmquist WF (2011c) The role of the breast cancer resistance protein (ABCG2) in the distribution of sorafenib to the brain. $J$ Pharmacol Exp Ther 336:223-233.

Aguzzi A, Brandner S, Sure U, Ruedi D and Isenmann S (1994) Transgenic and knockout mice: models of neurological disease. Brain Pathol 4:3-20.

Ahn JE, Karlsson MO, Dunne A and Ludden TM (2008) Likelihood based approaches to handling data below the quantification limit using NONMEM VI. $J$ Pharmacokinet Pharmacodyn 35:401-421.

Ahn SY, Eraly SA, Tsigelny I and Nigam SK (2009) Interaction of organic cations with organic anion transporters. $J$ Biol Chem 284:31422-31430.

Alcantara Llaguno S, Chen J, Kwon CH, Jackson EL, Li Y, Burns DK, Alvarez-Buylla A and Parada LF (2009) Malignant astrocytomas originate from neural stem/progenitor cells in a somatic tumor suppressor mouse model. Cancer Cell 15:45-56.

Amberger-Murphy V (2003) Glioma invasion: mechanism, modulation and future possibilities. Acta Neurochir 145:613-614.

Angeletti RH, Novikoff PM, Juvvadi SR, Fritschy JM, Meier PJ and Wolkoff AW (1997) The choroid plexus epithelium is the site of the organic anion transport protein in the brain. Proc Natl Acad Sci U S A 94:283-286. 
Antunes L, Angioi-Duprez KS, Bracard SR, Klein-Monhoven NA, Le Faou AE, Duprez AM and Plenat FM (2000) Analysis of tissue chimerism in nude mouse brain and abdominal xenograft models of human glioblastoma multiforme: what does it tell us about the models and about glioblastoma biology and therapy? J Histochem Cytochem 48:847-858.

Arndt P, Volk C, Gorboulev V, Budiman T, Popp C, Ulzheimer-Teuber I, Akhoundova A, Koppatz S, Bamberg E, Nagel G and Koepsell H (2001) Interaction of cations, anions, and weak base quinine with rat renal cation transporter rOCT2 compared with rOCT1. Am J Physiol Renal Physiol 281:454-468.

Ayuso-Sacido A, Moliterno JA, Kratovac S, Kapoor GS, O'Rourke DM, Holland EC, Garcia-Verdugo JM, Roy NS and Boockvar JA (2010) Activated EGFR signaling increases proliferation, survival, and migration and blocks neuronal differentiation in post-natal neural stem cells. J Neurooncol 97:323-337.

Barendt WM and Wright SH (2002) The human organic cation transporter (hOCT2) recognizes the degree of substrate ionization. J Biol Chem 277:22491-22496.

Baselga J (2006) Targeting tyrosine kinases in cancer: the second wave. Science 312:1175-1178.

Batchelor TT, Sorensen AG, di Tomaso E, Zhang WT, Duda DG, Cohen KS, Kozak KR, Cahill DP, Chen PJ, Zhu M, Ancukiewicz M, Mrugala MM, Plotkin S, Drappatz J, Louis DN, Ivy P, Scadden DT, Benner T, Loeffler JS, Wen PY and Jain RK (2007) AZD2171, a pan-VEGF receptor tyrosine kinase inhibitor, normalizes tumor vasculature and alleviates edema in glioblastoma patients. Cancer Cell 11:83-95.

Battegay EJ, Rupp J, Iruela-Arispe L, Sage EH and Pech M (1994) PDGF-BB modulates endothelial proliferation and angiogenesis in vitro via PDGF beta-receptors. $J$ Cell Biol 125:917-928.

Beal S and Sheiner L (1980) The Nonmem System. Am Stat 34:118-119.

Beaulieu E, Demeule M, Ghitescu L and Beliveau R (1997) P-glycoprotein is strongly expressed in the luminal membranes of the endothelium of blood vessels in the brain. Biochem J 326:539-544.

Becher OJ and Holland EC (2006) Genetically engineered models have advantages over xenografts for preclinical studies. Cancer Res 66:3355-3358.

Bello L, Giussani C, Carrabba G, Pluderi M, Costa F and Bikfalvi A (2004) Angiogenesis and invasion in gliomas. Cancer Treat Res 117:263-284. 
Bendayan R, Lee G and Bendayan M (2002) Functional expression and localization of P-glycoprotein at the blood brain barrier. Microsc Res Techniq 57:365-380.

Bendszus M, Warmuth-Metz M, Klein R, Burger R, Schichor C, Tonn JC and Solymosi L (2000) MR spectroscopy in gliomatosis cerebri. AJNR American journal of neuroradiology 21:375-380.

Benfeldt E and Groth L (1998) Feasibility of measuring lipophilic or protein-bound drugs in the dermis by in vivo microdialysis after topical or systemic drug administration. Acta Derm Venereol 78:274-278.

Benveniste H, Drejer J, Schousboe A and Diemer NH (1984) Elevation of the extracellular concentrations of glutamate and aspartate in rat hippocampus during transient cerebral ischemia monitored by intracerebral microdialysis. $J$ Neurochem 43:1369-1374.

Benveniste H, Drejer J, Schousboe A and Diemer NH (1987) Regional cerebral glucose phosphorylation and blood flow after insertion of a microdialysis fiber through the dorsal hippocampus in the rat. $J$ Neurochem 49:729-734.

Benveniste H and Huttemeier PC (1990) Microdialysis--theory and application. Prog Neurobiol 35:195-215.

Bergers $\mathrm{G}$ and Benjamin LE (2003) Tumorigenesis and the angiogenic switch. Nat Rev Cancer 3:401-410.

Bert L, Favale D, Jego G, Greve P, Guilloux JP, Guiard BP, Gardier AM, Suaud-Chagny MF and Lestage P (2004) Rapid and precise method to locate microdialysis probe implantation in the rodent brain. J Neurosci Methods 140:53-57.

Blakeley JO, Olson J, Grossman SA, He X, Weingart J and Supko JG (2009) Effect of blood brain barrier permeability in recurrent high grade gliomas on the intratumoral pharmacokinetics of methotrexate: a microdialysis study. $J$ Neurooncol 91:51-58.

Blaney SM, Takimoto C, Murry DJ, Kuttesch N, McCully C, Cole DE, Godwin K and Balis FM (1998) Plasma and cerebrospinal fluid pharmacokinetics of 9-aminocamptothecin (9-AC), irinotecan (CPT-11), and SN-38 in nonhuman primates. Cancer ChemotherPharmacol 41:464-468.

Bleasby K, Castle JC, Roberts CJ, Cheng C, Bailey WJ, Sina JF, Kulkarni AV, Hafey MJ, Evers R, Johnson JM, Ulrich RG and Slatter JG (2006) Expression profiles of 50 xenobiotic transporter genes in humans and pre-clinical species: a resource for investigations into drug disposition. Xenobiotica 36:963-988. 
Boschi G and Scherrmann J (2000) Microdialysis in mice for drug delivery research. AdvDrug DelivRev 45:271-281.

Brastianos PK and Batchelor TT (2010) Vascular endothelial growth factor inhibitors in malignant gliomas. Targeted oncology 5:167-174.

Bredel M, Pollack IF, Hamilton RL and James CD (1999) Epidermal growth factor receptor expression and gene amplification in high-grade non-brainstem gliomas of childhood. Clin Cancer Res 5:1786-1792.

Bronger H, Konig J, Kopplow K, Steiner HH, Ahmadi R, Herold-Mende C, Keppler D and Nies AT (2005) ABCC drug efflux pumps and organic anion uptake transporters in human gliomas and the blood-tumor barrier. Cancer Res 65:1141911428.

Broniscer A, Baker SJ, Stewart CF, Merchant TE, Laningham FH, Schaiquevich P, Kocak M, Morris EB, Endersby R, Ellison DW and Gajjar A (2009) Phase I and pharmacokinetic studies of erlotinib administered concurrently with radiotherapy for children, adolescents, and young adults with high-grade glioma. Clin Cancer Res 15:701-707.

Broniscer A, Baker SJ, West AN, Fraser MM, Proko E, Kocak M, Dalton J, Zambetti GP, Ellison DW, Kun LE, Gajjar A, Gilbertson RJ and Fuller CE (2007a) Clinical and molecular characteristics of malignant transformation of low-grade glioma in children. J Clin Oncol 25:682-689.

Broniscer A and Gajjar A (2004) Supratentorial high-grade astrocytoma and diffuse brainstem glioma: two challenges for the pediatric oncologist. Oncologist 9:197206.

Broniscer A, Iacono L, Chintagumpala M, Fouladi M, Wallace D, Bowers DC, Stewart C, Krasin MJ and Gajjar A (2005) Role of temozolomide after radiotherapy for newly diagnosed diffuse brainstem glioma in children: results of a multiinstitutional study (SJHG-98). Cancer 103:133-139.

Broniscer A, Panetta JC, O'Shaughnessy M, Fraga C, Bai F, Krasin MJ, Gajjar A and Stewart CF (2007b) Plasma and cerebrospinal fluid pharmacokinetics of erlotinib and its active metabolite OSI-420. Clin Cancer Res 13:1511-1515.

Broniscer A, Panetta JC, O'Shaughnessy M, Fraga C, Bai F, Krasin MJ, Gajjar A and Stewart CF (2007c) Plasma and cerebrospinal fluid pharmacokinetics of erlotinib and its active metabolite OSI-420. Clin Cancer Res 13:1511-1515.

Brown JM and Wilson WR (2004) Exploiting tumour hypoxia in cancer treatment. Nat Rev Cancer 4:437-447. 
Brown PD, Krishnan S, Sarkaria JN, Wu W, Jaeckle KA, Uhm JH, Geoffroy FJ, Arusell R, Kitange G, Jenkins RB, Kugler JW, Morton RF, Rowland KM, Jr., Mischel P, Yong WH, Scheithauer BW, Schiff D, Giannini C and Buckner JC (2008) Phase I/II trial of erlotinib and temozolomide with radiation therapy in the treatment of newly diagnosed glioblastoma multiforme: North Central Cancer Treatment Group Study N0177. J Clin Oncol 26:5603-5609.

Bruce JN and Oldfield EH (1988) Method for sequential sampling of cerebrospinal fluid in humans. Neurosurgery 23:788-790.

Brunner M and Langer O (2006) Microdialysis versus other techniques for the clinical assessment of in vivo tissue drug distribution. AAPS J 8:263-271.

Calatozzolo C, Gelati M, Ciusani E, Sciacca FL, Pollo B, Cajola L, Marras C, Silvani A, Vitellaro-Zuccarello L, Croci D, Boiardi A and Salmaggi A (2005) Expression of drug resistance proteins Pgp, MRP1, MRP3, MRP5 and GST-pi in human glioma. J Neurooncol 74:113-121.

Camphausen K, Purow B, Sproull M, Scott T, Ozawa T, Deen DF and Tofilon PJ (2005a) Influence of in vivo growth on human glioma cell line gene expression: convergent profiles under orthotopic conditions. Proc Natl Acad Sci USA 102:8287-8292.

Camphausen K, Purow B, Sproull M, Scott T, Ozawa T, Deen DF and Tofilon PJ (2005b) Orthotopic growth of human glioma cells quantitatively and qualitatively influences radiation-induced changes in gene expression. Cancer Res 65:1038910393.

Carcaboso AM, Elmeliegy MA, Shen J, Juel SJ, Zhang ZM, Calabrese C, Tracey L, Waters CM and Stewart CF (2010) Tyrosine kinase inhibitor gefitinib enhances topotecan penetration of gliomas. Cancer Res 70:4499-4508.

Carmeliet P and Jain RK (2000) Angiogenesis in cancer and other diseases. Nature 407:249-257.

Carneheim C and Stahle L (1991) Microdialysis of lipophilic compounds: a methodological study. Pharmacol Toxicol 69:378-380.

CBTRUS (2010) Primary Brain and Central Nervous System Tumors Diagnosed in the United States in 2004-2006, Statistical Report, Central Brain Tumor Registry of the United States. 
Chaurasia CS, Muller M, Bashaw ED, Benfeldt E, Bolinder J, Bullock R, Bungay PM, DeLange EC, Derendorf H, Elmquist WF, Hammarlund-Udenaes M, Joukhadar C, Kellogg DL, Jr., Lunte CE, Nordstrom CH, Rollema H, Sawchuk RJ, Cheung BW, Shah VP, Stahle L, Ungerstedt U, Welty DF and Yeo H (2007) AAPS-FDA workshop white paper: microdialysis principles, application and regulatory perspectives. Pharm Res 24:1014-1025.

Chen Y, Agarwal S, Shaik NM, Chen C, Yang Z and Elmquist WF (2009) P-glycoprotein and breast cancer resistance protein influence brain distribution of dasatinib. $J$ Pharmacol Exp Ther 330:956-963.

Cheng SY, Huang HJ, Nagane M, Ji XD, Wang D, Shih CC, Arap W, Huang CM and Cavenee WK (1996) Suppression of glioblastoma angiogenicity and tumorigenicity by inhibition of endogenous expression of vascular endothelial growth factor. Proc Natl Acad Sci US A 93:8502-8507.

Choudhuri S, Cherrington NJ, Li N and Klaassen CD (2003) Constitutive expression of various xenobiotic and endobiotic transporter mRNAs in the choroid plexus of rats. Drug Metab Dispos 31:1337-1345.

Chow LM and Baker SJ (2012) Capturing the molecular and biological diversity of highgrade astrocytoma in genetically engineered mouse models. Oncotarget 3:67-77.

Chow LM, Endersby R, Zhu X, Rankin S, Qu C, Zhang J, Broniscer A, Ellison DW and Baker SJ (2011) Cooperativity within and among Pten, p53, and Rb pathways induces high-grade astrocytoma in adult brain. Cancer Cell 19:305-316.

Ciardiello F, Caputo R, Bianco R, Damiano V, Fontanini G, Cuccato S, De Placido S, Bianco AR and Tortora G (2001) Inhibition of growth factor production and angiogenesis in human cancer cells by ZD1839 (Iressa), a selective epidermal growth factor receptor tyrosine kinase inhibitor. Clin Cancer Res 7:1459-1465.

Cisternino S, Mercier C, Bourasset F, Roux F and Scherrmann JM (2004) Expression, up-regulation, and transport activity of the multidrug-resistance protein Abcg2 at the mouse blood-brain barrier. Cancer Res 64:3296-3301.

Cooray HC, Blackmore CG, Maskell L and Barrand MA (2002) Localisation of breast cancer resistance protein in microvessel endothelium of human brain. Neuroreport 13:2059-2063.

Cordon-Cardo C, O'Brien JP, Casals D, Rittman-Grauer L, Biedler JL, Melamed MR and Bertino JR (1989) Multidrug-resistance gene (P-glycoprotein) is expressed by endothelial cells at blood-brain barrier sites. Proc Natl Acad Sci U S A 86:695698. 
D'argenio DZ, Schumitzky A and Wang X (2009) ADAPT 5 User's Guide:

Pharmacokinetic/ Pharmacodynamic Systems Analysis Software, Los Angeles. http://bmsr.usc.edu/Software/ADAPT/ADAPT.html (accessed Januray 15, 2010).

Daood M, Tsai C, Ahdab-Barmada M and Watchko JF (2008) ABC transporter (P-gp/ABCB1, MRP1/ABCC1, BCRP/ABCG2) expression in the developing human CNS. Neuropediatrics 39:211-218.

de Lange EC (2004) Potential role of ABC transporters as a detoxification system at the blood-CSF barrier. AdvDrug DelivRev 56:1793-1809.

de Lange EC, Danhof M, de Boer AG and Breimer DD (1994) Critical factors of intracerebral microdialysis as a technique to determine the pharmacokinetics of drugs in rat brain. Brain Res 666:1-8.

de Lange EC, Danhof M, de Boer AG and Breimer DD (1997) Methodological considerations of intracerebral microdialysis in pharmacokinetic studies on drug transport across the blood-brain barrier. Brain Res Brain Res Rev 25:27-49.

de Lange EC, de Bock G, Schinkel AH, de Boer AG and Breimer DD (1998) BBB transport and P-glycoprotein functionality using MDR1A (-/-) and wild-type mice. Total brain versus microdialysis concentration profiles of rhodamine-123. Pharm Res 15:1657-1665.

de Lange EC, de Boer AG and Breimer DD (2000) Methodological issues in microdialysis sampling for pharmacokinetic studies. AdvDrug DelivRev 45:125148.

de Lange ECM and Danhof M (2002) Considerations in the use of cerebrospinal fluid pharmacokinetics to predict brain target concentrations in the clinical setting Implications of the barriers between blood and brain. Clinical Pharmacokinetics 41:691-703.

de Vries NA, Beijnen JH and van Tellingen O (2009) High-grade glioma mouse models and their applicability for preclinical testing. Cancer Treat Rev 35:714-723.

de Vries NA, Bruggeman SW, Hulsman D, de Vries HI, Zevenhoven J, Buckle T, Hamans BC, Leenders WP, Beijnen JH, van Lohuizen M, Berns AJ and van Tellingen O (2010) Rapid and robust transgenic high-grade glioma mouse models for therapy intervention studies. Clin Cancer Res 16:3431-3441.

de Vries NA, Zhao J, Kroon E, Buckle T, Beijnen JH and van Tellingen O (2007) Pglycoprotein and breast cancer resistance protein: two dominant transporters working together in limiting the brain penetration of topotecan. Clin Cancer Res 13:6440-6449. 
Decleves X, Amiel A, Delattre JY and Scherrmann JM (2006) Role of ABC transporters in the chemoresistance of human gliomas. Curr Cancer Drug Targets 6:433-445.

Decleves X, Fajac A, Lehmann-Che J, Tardy M, Mercier C, Hurbain I, Laplanche JL, Bernaudin JF and Scherrmann JM (2002) Molecular and functional MDR1-Pgp and MRPs expression in human glioblastoma multiforme cell lines. IntJCancer 98:173-180.

Degorter MK, Xia CQ, Yang JJ and Kim RB (2011) Drug Transporters in Drug Efficacy and Toxicity. Annu Rev Pharmacol Toxicol. 52:249-273.

Demeule M, Shedid D, Beaulieu E, Del Maestro RF, Moghrabi A, Ghosn PB, Moumdjian R, Berthelet F and Beliveau R (2001) Expression of multidrugresistance P-glycoprotein (MDR1) in human brain tumors. Int J Cancer 93:62-66.

Dickson PV, Hamner B, Ng CY, Hall MM, Zhou J, Hargrove PW, McCarville MB and Davidoff AM (2007) In vivo bioluminescence imaging for early detection and monitoring of disease progression in a murine model of neuroblastoma. $J$ Pediatr Surg 42:1172-1179.

Ding H, Roncari L, Shannon P, Wu X, Lau N, Karaskova J, Gutmann DH, Squire JA, Nagy A and Guha A (2001) Astrocyte-specific expression of activated p21-ras results in malignant astrocytoma formation in a transgenic mouse model of human gliomas. Cancer Res 61:3826-3836.

Doolittle ND, Peereboom DM, Christoforidis GA, Hall WA, Palmieri D, Brock PR, Campbell KC, Dickey DT, Muldoon LL, O'Neill BP, Peterson DR, Pollock B, Soussain C, Smith Q, Tyson RM and Neuwelt EA (2007) Delivery of chemotherapy and antibodies across the blood-brain barrier and the role of chemoprotection, in primary and metastatic brain tumors: report of the Eleventh Annual Blood-Brain Barrier Consortium meeting. J Neurooncol 81:81-91.

Dorr A, Sled JG and Kabani N (2007) Three-dimensional cerebral vasculature of the CBA mouse brain: a magnetic resonance imaging and micro computed tomography study. Neuroimage 35:1409-1423.

Doyle LA, Yang W, Abruzzo LV, Krogmann T, Gao Y, Rishi AK and Ross DD (1998) A multidrug resistance transporter from human MCF-7 breast cancer cells.

Proceedings of the National Academy of Sciences of the United States of America 95:15665-15670. 
Dresemann G, Weller M, Rosenthal MA, Wedding U, Wagner W, Engel E, Heinrich B, Mayer-Steinacker R, Karup-Hansen A, Fluge O, Nowak A, Mehdorn M, Schleyer E, Krex D, Olver IN, Steinbach JP, Hosius C, Sieder C, Sorenson G, Parker R and Nikolova Z (2010) Imatinib in combination with hydroxyurea versus hydroxyurea alone as oral therapy in patients with progressive pretreated glioblastoma resistant to standard dose temozolomide. J Neurooncol 96:393-402.

Duo J, Fletcher H and Stenken JA (2006) Natural and synthetic affinity agents as microdialysis sampling mass transport enhancers: current progress and future perspectives. Biosens Bioelectron 22:449-457.

Dvorak HF, Brown LF, Detmar M and Dvorak AM (1995) Vascular permeability factor/vascular endothelial growth factor, microvascular hyperpermeability, and angiogenesis. The American journal of pathology 146:1029-1039.

Dvorak HF, Nagy JA, Feng D, Brown LF and Dvorak AM (1999) Vascular permeability factor/vascular endothelial growth factor and the significance of microvascular hyperpermeability in angiogenesis. Curr Top Microbiol Immunol 237:97-132.

Eberhard A, Kahlert S, Goede V, Hemmerlein B, Plate KH and Augustin HG (2000) Heterogeneity of angiogenesis and blood vessel maturation in human tumors: implications for antiangiogenic tumor therapies. Cancer Res 60:1388-1393.

Elmeliegy MA, Carcaboso AM, LM LC, Zhang ZM, Calabrese C, Throm SL, Wang F, Baker SJ and Stewart CF (2011a) Magnetic resonance imaging-guided microdialysis cannula implantation in a spontaneous high-grade glioma murine model. J Pharm Sci 100:4210-4214.

Elmeliegy MA, Carcaboso AM, Tagen M, Bai F and Stewart CF (2011b) Role of ATP-binding cassette and solute carrier transporters in erlotinib CNS penetration and intracellular accumulation. Clin Cancer Res 17:89-99.

Eros D, Kovesdi I, Orfi L, Takacs-Novak K, Acsady G and Keri G (2002) Reliability of $\log \mathrm{P}$ predictions based on calculated molecular descriptors: a critical review. Curr Med Chem 9:1819-1829.

Fan QW and Weiss WA (2010) Targeting the RTK-PI3K-mTOR axis in malignant glioma: overcoming resistance. Curr Top Microbiol Immunol 347:279-296.

Fattori S, Becherini F, Cianfriglia M, Parenti G, Romanini A and Castagna M (2007) Human brain tumors: multidrug-resistance P-glycoprotein expression in tumor cells and intratumoral capillary endothelial cells. Virchows Arch 451:81-87.

Ferrara N (2004) Vascular endothelial growth factor as a target for anticancer therapy. Oncologist 9:2-10. 
Filipski KK, Loos WJ, Verweij J and Sparreboom A (2008) Interaction of Cisplatin with the human organic cation transporter 2. Clin Cancer Res 14:3875-3880.

Fine RL, Chen J, Balmaceda C, Bruce JN, Huang M, Desai M, Sisti MB, McKhann GM, Goodman RR, Bertino JS, Jr., Nafziger AN and Fetell MR (2006) Randomized study of paclitaxel and tamoxifen deposition into human brain tumors: implications for the treatment of metastatic brain tumors. Clin Cancer Res 12:5770-5776.

Finlay JL, Boyett JM, Yates AJ, Wisoff JH, Milstein JM, Geyer JR, Bertolone SJ, McGuire P, Cherlow JM, Tefft M and et al. (1995) Randomized phase III trial in childhood high-grade astrocytoma comparing vincristine, lomustine, and prednisone with the eight-drugs-in-1-day regimen. Childrens Cancer Group. $J$ Clin Oncol 13:112-123.

Friedman HS, Prados MD, Wen PY, Mikkelsen T, Schiff D, Abrey LE, Yung WK, Paleologos N, Nicholas MK, Jensen R, Vredenburgh J, Huang J, Zheng M and Cloughesy $\mathrm{T}$ (2009) Bevacizumab alone and in combination with irinotecan in recurrent glioblastoma. J Clin Oncol 27:4733-4740.

Friesel RE and Maciag T (1995) Molecular mechanisms of angiogenesis: fibroblast growth factor signal transduction. FASEB J 9:919-925.

Frosina G (2009) DNA repair and resistance of gliomas to chemotherapy and radiotherapy. Mol Cancer Res 7:989-999.

Fukumura D and Jain RK (2007) Tumor microvasculature and microenvironment: targets for anti-angiogenesis and normalization. Microvasc Res 74:72-84.

Fuller GN (2008) The WHO Classification of Tumours of the Central Nervous System, 4th edition. Arch Pathol Lab Med 132:906-906.

Furnari FB, Fenton T, Bachoo RM, Mukasa A, Stommel JM, Stegh A, Hahn WC, Ligon KL, Louis DN, Brennan C, Chin L, DePinho RA and Cavenee WK (2007) Malignant astrocytic glioma: genetics, biology, and paths to treatment. Genes Dev 21:2683-2710.

Gao B, Hagenbuch B, Kullak-Ublick GA, Benke D, Aguzzi A and Meier PJ (2000) Organic anion-transporting polypeptides mediate transport of opioid peptides across blood-brain barrier. J Pharmacol Exp Ther 294:73-79.

Gao B, Stieger B, Noe B, Fritschy JM and Meier PJ (1999) Localization of the organic anion transporting polypeptide 2 (Oatp2) in capillary endothelium and choroid plexus epithelium of rat brain. J Histochem Cytochem 47:1255-1264. 
Geoerger B, Hargrave D, Thomas F, Ndiaye A, Frappaz D, Andreiuolo F, Varlet P, Aerts I, Riccardi R, Jaspan T, Chatelut E, Le Deley MC, Paoletti X, Saint-Rose C, Leblond P, Morland B, Gentet JC, Meresse V and Vassal G (2011) Innovative Therapies for Children with Cancer pediatric phase I study of erlotinib in brainstem glioma and relapsing/refractory brain tumors. Neuro Oncol 13:109-118.

Gerhardt H and Betsholtz C (2003) Endothelial-pericyte interactions in angiogenesis. Cell Tissue Res 314:15-23.

Gerstner ER and Fine RL (2007) Increased permeability of the blood-brain barrier to chemotherapy in metastatic brain tumors: establishing a treatment paradigm. $J$ Clin Oncol 25:2306-2312.

Giacomini KM, Huang SM, Tweedie DJ, Benet LZ, Brouwer KL, Chu X, Dahlin A, Evers R, Fischer V, Hillgren KM, Hoffmaster KA, Ishikawa T, Keppler D, Kim RB, Lee CA, Niemi M, Polli JW, Sugiyama Y, Swaan PW, Ware JA, Wright SH, Yee SW, Zamek-Gliszczynski MJ and Zhang L (2010) Membrane transporters in drug development. Nature Reviews Drug Discovery 9:215-236.

Giese A, Bjerkvig R, Berens ME and Westphal M (2003) Cost of migration: invasion of malignant gliomas and implications for treatment. J Clin Oncol 21:1624-1636.

Gilbertson RJ, Hill DA, Hernan R, Kocak M, Geyer R, Olson J, Gajjar A, Rush L, Hamilton RL, Finkelstein SD and Pollack IF (2003) ERBB1 is amplified and overexpressed in high-grade diffusely infiltrative pediatric brain stem glioma. Clin Cancer Res 9:3620-3624.

Gilbertson RJ and Rich JN (2007) Making a tumour's bed: glioblastoma stem cells and the vascular niche. Nat Rev Cancer 7:733-736.

Gillespie DL, Whang K, Ragel BT, Flynn JR, Kelly DA and Jensen RL (2007) Silencing of hypoxia inducible factor-1alpha by RNA interference attenuates human glioma cell growth in vivo. Clin Cancer Res 13:2441-2448.

Gossen M and Bujard H (1992) Tight control of gene expression in mammalian cells by tetracycline-responsive promoters. Proc Natl Acad Sci U S A 89:5547-5551.

Gottesman MM (1993) How cancer cells evade chemotherapy: sixteenth Richard and Hinda Rosenthal Foundation Award Lecture. Cancer Res 53:747-754.

Gould S and Scott RC (2005) 2-Hydroxypropyl-beta-cyclodextrin (HP-beta-CD): a toxicology review. Food Chem Toxicol 43:1451-1459.

Guha A, Dashner K, Black PM, Wagner JA and Stiles CD (1995) Expression of PDGF and PDGF receptors in human astrocytoma operation specimens supports the existence of an autocrine loop. Int J Cancer 60:168-173. 
Guha A, Feldkamp MM, Lau N, Boss G and Pawson A (1997) Proliferation of human malignant astrocytomas is dependent on Ras activation. Oncogene 15:2755-2765.

Guo P, Hu B, Gu W, Xu L, Wang D, Huang HJ, Cavenee WK and Cheng SY (2003) Platelet-derived growth factor-B enhances glioma angiogenesis by stimulating vascular endothelial growth factor expression in tumor endothelia and by promoting pericyte recruitment. The American journal of pathology 162:10831093.

Gururangan S, Chi SN, Young Poussaint T, Onar-Thomas A, Gilbertson RJ, Vajapeyam S, Friedman HS, Packer RJ, Rood BN, Boyett JM and Kun LE (2010) Lack of efficacy of bevacizumab plus irinotecan in children with recurrent malignant glioma and diffuse brainstem glioma: a Pediatric Brain Tumor Consortium study. J Clin Oncol 28:3069-3075.

Halatsch ME, Gehrke EE, Vougioukas VI, Botefur IC, F AB, Efferth T, Gebhart E, Domhof S, Schmidt U and Buchfelder M (2004) Inverse correlation of epidermal growth factor receptor messenger RNA induction and suppression of anchorageindependent growth by OSI-774, an epidermal growth factor receptor tyrosine kinase inhibitor, in glioblastoma multiforme cell lines. J Neurosurg 100:523-533.

Hammarlund-Udenaes M (2010) Active-site concentrations of chemicals - are they a better predictor of effect than plasma/organ/tissue concentrations? Basic Clin Pharmacol Toxicol 106:215-220.

Hammarlund-Udenaes M, Friden M, Syvanen S and Gupta A (2008) On the rate and extent of drug delivery to the brain. Pharm Res 25:1737-1750.

Hammarlund-Udenaes M, Paalzow LK and de Lange EC (1997) Drug equilibration across the blood-brain barrier--pharmacokinetic considerations based on the microdialysis method. PharmRes 14:128-134.

Hanahan D and Folkman J (1996) Patterns and emerging mechanisms of the angiogenic switch during tumorigenesis. Cell 86:353-364.

Hatanpaa KJ, Burma S, Zhao D and Habib AA (2010) Epidermal growth factor receptor in glioma: signal transduction, neuropathology, imaging, and radioresistance. Neoplasia 12:675-684.

Hayer-Zillgen M, Bruss M and Bonisch H (2002) Expression and pharmacological profile of the human organic cation transporters hOCT1, hOCT2 and hOCT3. $\mathrm{Br} J$ Pharmacol 136:829-836.

Hediger MA, Romero MF, Peng JB, Rolfs A, Takanaga H and Bruford EA (2004) The ABCs of solute carriers: physiological, pathological and therapeutic implications of human membrane transport proteinsIntroduction. Pflugers Arch 447:465-468. 
Hegmann EJ, Bauer HC and Kerbel RS (1992) Expression and functional activity of P-glycoprotein in cultured cerebral capillary endothelial cells. Cancer Res 52:6969-6975.

Heimberger AB, Learn CA, Archer GE, McLendon RE, Chewning TA, Tuck FL, Pracyk JB, Friedman AH, Friedman HS, Bigner DD and Sampson JH (2002) Brain tumors in mice are susceptible to blockade of epidermal growth factor receptor (EGFR) with the oral, specific, EGFR-tyrosine kinase inhibitor ZD1839 (iressa). Clin Cancer Res 8:3496-3502.

Heyer J, Kwong LN, Lowe SW and Chin L (2010) Non-germline genetically engineered mouse models for translational cancer research. Nat Rev Cancer 10:470-480.

Hill KK, West SA, Ekhator NN, Bruce AB, Wortman MD, Baker DG and Geracioti TD, Jr. (1999) The effect of lumbar puncture stress on dopamine and serotonin metabolites in human cerebrospinal fluid. Neuroscience Letters 276:25-28.

Hofer S and Frei K (2007) Gefitinib concentrations in human glioblastoma tissue. $J$ Neurooncol 82:175-176.

Hofer S, Frei K and Rutz HP (2006) Gefitinib accumulation in glioblastoma tissue. Cancer Biol Ther 5:483-484.

Hori S, Ohtsuki S, Tachikawa M, Kimura N, Kondo T, Watanabe M, Nakashima E and Terasaki T (2004) Functional expression of rat ABCG2 on the luminal side of brain capillaries and its enhancement by astrocyte-derived soluble factor(s). $J$ Neurochem 90:526-536.

Houchens DP, Ovejera AA, Riblet SM and Slagel DE (1983) Human brain tumor xenografts in nude mice as a chemotherapy model. Eur J Cancer Clin Oncol 19:799-805.

Hsyu PH, Gisclon LG, Hui AC and Giacomini KM (1988) Interactions of organic anions with the organic cation transporter in renal BBMV. Am J Physiol 254:F56-61.

Hu S, Franke RM, Filipski KK, Hu C, Orwick SJ, de Bruijn EA, Burger H, Baker SD and Sparreboom A (2008) Interaction of imatinib with human organic ion carriers. Clin Cancer Res 14:3141-3148.

Huai-Yun H, Secrest DT, Mark KS, Carney D, Brandquist C, Elmquist WF and Miller DW (1998) Expression of multidrug resistance-associated protein (MRP) in brain microvessel endothelial cells. Biochem Biophys Res Commun 243:816-820.

Hyafil F, Vergely C, Du Vignaud P and Grand-Perret T (1993) In vitro and in vivo reversal of multidrug resistance by GF120918, an acridonecarboxamide derivative. Cancer Res 53:4595-4602. 
Italia A, Schiavi M and Ventura P (1990) Direct Liquid-Chromatographic Separation of Enantiomeric and Diastereomeric Terpenic Alcohols as Beta-Cyclodextrin Inclusion Complexes. Journal of Chromatography 503:266-271.

Jacobs VL, Valdes PA, Hickey WF and De Leo JA (2011) Current review of in vivo GBM rodent models: emphasis on the CNS-1 tumour model. ASN neuro 3:171181.

Jacobson I and Hamberger A (1985) Kainic Acid-Induced Changes of Extracellular Amino-Acid Levels, Evoked-Potentials and Eeg Activity in the Rabbit OlfactoryBulb. Brain Research 348:289-296.

Jacobson I, Sandberg M and Hamberger A (1985) Mass-Transfer in Brain Dialysis Devices - a New Method for the Estimation of Extracellular Amino-Acids Concentration. Journal of Neuroscience Methods 15:263-268.

Jain RK (2001) Delivery of molecular and cellular medicine to solid tumors. Adv Drug Deliv Rev 46:149-168.

Jain RK (2005) Normalization of tumor vasculature: an emerging concept in antiangiogenic therapy. Science 307:58-62.

Jain RK, di Tomaso E, Duda DG, Loeffler JS, Sorensen AG and Batchelor TT (2007) Angiogenesis in brain tumours. Nat Rev Neurosci 8:610-622.

Jensen SM, Hansen HS, Johansen T and Malmlof K (2007) In vivo and in vitro microdialysis sampling of free fatty acids. J Pharm Biomed Anal 43:1751-1756.

Johanson CE, Duncan JA, Stopa EG and Baird A (2005) Enhanced prospects for drug delivery and brain targeting by the choroid plexus-CSF route. PharmRes 22:10111037.

Johnson RD and Justice JB (1983) Model Studies for Brain Dialysis. Brain Research Bulletin 10:567-571.

Jonker JW, Wagenaar E, van Eijl S and Schinkel AH (2003) Deficiency in the organic cation transporters 1 and 2 (Oct1/Oct2 [Slc22a1/Slc22a2]) in mice abolishes renal secretion of organic cations. Molecular and Cellular Biology 23:7902-7908.

Jonkers J and Berns A (2002) Conditional mouse models of sporadic cancer. Nat Rev Cancer 2:251-265. 
Kaddoumi A, Choi SU, Kinman L, Whittington D, Tsai CC, Ho RJ, Anderson BD and Unadkat JD (2007) Inhibition of P-glycoprotein activity at the primate bloodbrain barrier increases the distribution of nelfinavir into the brain but not into the cerebrospinal fluid. Drug Metab Dispos 35:1459-1462.

Kamoun WS, Ley CD, Farrar CT, Duyverman AM, Lahdenranta J, Lacorre DA, Batchelor TT, di Tomaso E, Duda DG, Munn LL, Fukumura D, Sorensen AG and Jain RK (2009) Edema Control by Cediranib, a Vascular Endothelial Growth Factor Receptor-Targeted Kinase Inhibitor, Prolongs Survival Despite Persistent Brain Tumor Growth in Mice. J Clin Oncol 15:2542:2552.

Karbach U, Kricke J, Meyer-Wentrup F, Gorboulev V, Volk C, Loffing-Cueni D, Kaissling B, Bachmann S and Koepsell H (2000) Localization of organic cation transporters OCT1 and OCT2 in rat kidney. Am J Physiol Renal Physiol 279:679687.

Karpel-Massler G, Wirtz CR and Halatsch ME (2011) Drug Combinations Enhancing the Antineoplastic Effects of Erlotinib in High-Grade Glioma. Recent Pat Anticancer Drug Discov 6:384-394.

Khramov AN and Stenken JA (1999a) Enhanced Microdialysis Extraction Efficiency of Ibuprofen in Vitro by Facilitated Transport with beta-Cyclodextrin. Anal Chem 71:1257-1264.

Khramov AN and Stenken JA (1999b) Enhanced microdialysis recovery of some tricyclic antidepressants and structurally related drugs by cyclodextrin-mediated transport. Analyst 124:1027-1033.

Kikuchi R, Kusuhara H, Sugiyama D and Sugiyama Y (2003) Contribution of organic anion transporter 3 (Slc22a8) to the elimination of p-aminohippuric acid and benzylpenicillin across the blood-brain barrier. J Pharmacol Exp Ther 306:51-58.

Kilic T, Alberta JA, Zdunek PR, Acar M, Iannarelli P, O'Reilly T, Buchdunger E, Black PM and Stiles CD (2000) Intracranial inhibition of platelet-derived growth factormediated glioblastoma cell growth by an orally active kinase inhibitor of the 2phenylaminopyrimidine class. Cancer Res 60:5143-5150.

Kim KJ, Li B, Winer J, Armanini M, Gillett N, Phillips HS and Ferrara N (1993) Inhibition of vascular endothelial growth factor-induced angiogenesis suppresses tumour growth in vivo. Nature 362:841-844.

Kniesel U and Wolburg H (2000) Tight junctions of the blood-brain barrier. Cell Mol Neurobiol 20:57-76. 
Kodaira H, Kusuhara H, Ushiki J, Fuse E and Sugiyama Y (2010) Kinetic analysis of the cooperation of P-glycoprotein (P-gp/Abcb1) and breast cancer resistance protein (Bcrp/Abcg2) in limiting the brain and testis penetration of erlotinib, flavopiridol, and mitoxantrone. $J$ Pharmacol Exp Ther 333:788-796.

Koepsell H and Endou H (2004) The SLC22 drug transporter family. Pflugers Arch 447:666-676.

Krakstad C and Chekenya M (2010) Survival signalling and apoptosis resistance in glioblastomas: opportunities for targeted therapeutics. Mol Cancer 9:135.

Kruijtzer CM, Beijnen JH, Rosing H, ten Bokkel Huinink WW, Schot M, Jewell RC, Paul EM and Schellens JH (2002) Increased oral bioavailability of topotecan in combination with the breast cancer resistance protein and P-glycoprotein inhibitor GF120918. J Clin Oncol 20:2943-2950.

Kucherlapati R (2012) Genetically modified mouse models for biomarker discovery and preclinical drug testing. Clin Cancer Res 18:625-630.

Kurosaki Y, Nakamura S, Shiojiri Y and Kawasaki H (1998) Lipo-microdialysis: a new microdialysis method for studying the pharmacokinetics of lipophilic substances. Biol Pharm Bull 21:194-196.

Kusuhara H, Sekine T, Utsunomiya-Tate N, Tsuda M, Kojima R, Cha SH, Sugiyama Y, Kanai Y and Endou H (1999) Molecular cloning and characterization of a new multispecific organic anion transporter from rat brain. J Biol Chem 274:1367513680 .

Kusuhara H and Sugiyama Y (2004) Efflux transport systems for organic anions and cations at the blood-CSF barrier. Adv Drug Deliv Rev 56:1741-1763.

Kusuhara H and Sugiyama Y (2005) Active efflux across the blood-brain barrier: role of the solute carrier family. NeuroRx 2:73-85.

Larjavaara S, Mantyla R, Salminen T, Haapasalo H, Raitanen J, Jaaskelainen J and Auvinen A (2007) Incidence of gliomas by anatomic location. Neuro Oncol 9:319-325.

Lassman AB, Rossi MR, Raizer JJ, Abrey LE, Lieberman FS, Grefe CN, Lamborn K, Pao W, Shih AH, Kuhn JG, Wilson R, Nowak NJ, Cowell JK, DeAngelis LM, Wen P, Gilbert MR, Chang S, Yung WA, Prados M and Holland EC (2005) Molecular study of malignant gliomas treated with epidermal growth factor receptor inhibitors: tissue analysis from North American Brain Tumor Consortium Trials 01-03 and 00-01. Clin Cancer Res 11:7841-7850. 
Lee G, Dallas S, Hong M and Bendayan R (2001) Drug transporters in the central nervous system: brain barriers and brain parenchyma considerations. Pharmacol Rev 53:569-596.

Lee J, Kotliarova S, Kotliarov Y, Li A, Su Q, Donin NM, Pastorino S, Purow BW, Christopher N, Zhang W, Park JK and Fine HA (2006) Tumor stem cells derived from glioblastomas cultured in bFGF and EGF more closely mirror the phenotype and genotype of primary tumors than do serum-cultured cell lines. Cancer Cell 9:391-403.

Leggas M, Adachi M, Scheffer GL, Sun D, Wielinga P, Du G, Mercer KE, Zhuang Y, Panetta JC, Johnston B, Scheper RJ, Stewart CF and Schuetz JD (2004a) Mrp4 confers resistance to topotecan and protects the brain from chemotherapy. Mol Cell Biol 24:7612-7621.

Leggas M, Zhuang Y, Welden J, Self Z, Waters CM and Stewart CF (2004b) Microbore HPLC method with online microdialysis for measurement of topotecan lactone and carboxylate in murine CSF. J Pharm Sci 93:2284-2295.

Legler JM, Ries LA, Smith MA, Warren JL, Heineman EF, Kaplan RS and Linet MS (1999) Cancer surveillance series [corrected]: brain and other central nervous system cancers: recent trends in incidence and mortality. J Natl Cancer Inst 91:1382-1390.

Lemos C, Jansen G and Peters GJ (2008) Drug transporters: recent advances concerning BCRP and tyrosine kinase inhibitors. British Journal of Cancer 98:857-862.

Leon SP, Folkerth RD and Black PM (1996) Microvessel density is a prognostic indicator for patients with astroglial brain tumors. Cancer 77:362-372.

Lewis NL, Lewis LD, Eder JP, Reddy NJ, Guo F, Pierce KJ, Olszanski AJ and Cohen RB (2009) Phase I study of the safety, tolerability, and pharmacokinetics of oral CP-868,596, a highly specific platelet-derived growth factor receptor tyrosine kinase inhibitor in patients with advanced cancers. J Clin Oncol 27:5262-5269.

Liang ML, Ma J, Ho M, Solomon L, Bouffet E, Rutka JT and Hawkins C (2008) Tyrosine kinase expression in pediatric high grade astrocytoma. $J$ Neurooncol 87:247-253.

Lin CJ, Tai Y, Huang MT, Tsai YF, Hsu HJ, Tzen KY and Liou HH (2010) Cellular localization of the organic cation transporters, OCT1 and OCT2, in brain microvessel endothelial cells and its implication for MPTP transport across the blood-brain barrier and MPTP-induced dopaminergic toxicity in rodents. $J$ Neurochem 114:717-727. 
Lin JH (2008) CSF as a surrogate for assessing CNS exposure: An industrial perspective. Current Drug Metabolism 9:46-59.

Lindberger M, Tomson T and Lars S (2002) Microdialysis sampling of carbamazepine, phenytoin and phenobarbital in subcutaneous extracellular fluid and subdural cerebrospinal fluid in humans: an in vitro and in vivo study of adsorption to the sampling device. Pharmacol Toxicol 91:158-165.

Lindefors N, Amberg G and Ungerstedt U (1989) Intracerebral Microdialysis .1. Experimental Studies of Diffusion Kinetics. Journal of Pharmacological Methods 22:141-156.

Ling J, Johnson KA, Miao Z, Rakhit A, Pantze MP, Hamilton M, Lum BL and Prakash C (2006) Metabolism and excretion of erlotinib, a small molecule inhibitor of epidermal growth factor receptor tyrosine kinase, in healthy male volunteers. Drug Metab Dispos 34:420-426.

Liu KW, Hu B and Cheng SY (2011) Platelet-derived growth factor receptor alpha in glioma: a bad seed. Chin J Cancer 30:590-602.

Loftsson T and Brewster ME (1996) Pharmaceutical applications of cyclodextrins. 1. Drug solubilization and stabilization. J Pharm Sci 85:1017-1025.

Loos WJ, Zamboni WC, Engels FK, de Bruijn P, Lam MH, de Wit R, Verweij J and Wiemer EA (2007) Pitfalls of the application of microdialysis in clinical oncology: controversial findings with docetaxel. J Pharm Biomed Anal 45:288294.

Lorico A, Rappa G, Finch RA, Yang D, Flavell RA and Sartorelli AC (1997) Disruption of the murine MRP (multidrug resistance protein) gene leads to increased sensitivity to etoposide (VP-16) and increased levels of glutathione. Cancer Res 57:5238-5242.

Loscher W and Potschka H (2005) Drug resistance in brain diseases and the role of drug efflux transporters. Nat Rev Neurosci 6:591-602.

Ma J, Li S, Reed K, Guo P and Gallo JM (2003) Pharmacodynamic-mediated effects of the angiogenesis inhibitor SU5416 on the tumor disposition of temozolomide in subcutaneous and intracerebral glioma xenograft models. J Pharmacol Exp Ther 305:833-839.

Ma J, Pulfer S, Li S, Chu J, Reed K and Gallo JM (2001) Pharmacodynamic-mediated reduction of temozolomide tumor concentrations by the angiogenesis inhibitor TNP-470. Cancer Res 61:5491-5498. 
Maeda T, Takahashi K, Ohtsu N, Oguma T, Ohnishi T, Atsumi R and Tamai I (2007) Identification of influx transporter for the quinolone antibacterial agent levofloxacin. Mol Pharm 4:85-94.

Maguire JH (1987) Some structural requirements for resolution of hydantoin enantiomers with a beta-cyclodextrin liquid chromatography column. J Chromatogr 387:453458.

Mahar Doan KM, Humphreys JE, Webster LO, Wring SA, Shampine LJ, Serabjit-Singh CJ, Adkison KK and Polli JW (2002) Passive permeability and P-glycoproteinmediated efflux differentiate central nervous system (CNS) and non-CNS marketed drugs. J Pharmacol Exp Ther 303:1029-1037.

Mahboobi S, Sellmer A, Eswayah A, Elz S, Uecker A and Bohmer FD (2008) Inhibition of PDGFR tyrosine kinase activity by a series of novel N-(3-(4-(pyridin-3-yl)-1Himidazol-2-ylamino)phenyl)amides: a SAR study on the bioisosterism of pyrimidine and imidazole. Eur J Med Chem 43:1444-1453.

Maliepaard M, van Gastelen MA, Tohgo A, Hausheer FH, van Waardenburg RC, de Jong LA, Pluim D, Beijnen JH and Schellens JH (2001) Circumvention of breast cancer resistance protein (BCRP)-mediated resistance to camptothecins in vitro using non-substrate drugs or the BCRP inhibitor GF120918. Clin Cancer Res 7:935-941.

Marchetti S, de Vries NA, Buckle T, Bolijn MJ, van Eijndhoven MA, Beijnen JH, Mazzanti R, van Tellingen O and Schellens JH (2008) Effect of the ATP-binding cassette drug transporters ABCB1, ABCG2, and ABCC2 on erlotinib hydrochloride (Tarceva) disposition in in vitro and in vivo pharmacokinetic studies employing $\mathrm{Bcrp}^{-/ /} / \mathrm{Mdrla} / 1 \mathrm{~b}^{-/-}$(triple-knockout) and wild-type mice. $\mathrm{Mol}$ Cancer Ther 7:2280-2287.

Marumoto T, Tashiro A, Friedmann-Morvinski D, Scadeng M, Soda Y, Gage FH and Verma IM (2009) Development of a novel mouse glioma model using lentiviral vectors. Nat Med 15:110-116.

Maurer TS, Debartolo DB, Tess DA and Scott DO (2005) Relationship between exposure and nonspecific binding of thirty-three central nervous system drugs in mice. Drug Metab Dispos 33:175-181.

Mauro A, Bulfone A, Turco E and Schiffer D (1991) Coexpression of platelet-derived growth factor (PDGF) B chain and PDGF B-type receptor in human gliomas. Childs Nerv Syst 7:432-436.

Mayer S, Maickel RP and Brodie BB (1959) Kinetics of Penetration of Drugs and Other Foreign Compounds into Cerebrospinal Fluid and Brain. Journal of Pharmacology and Experimental Therapeutics 127:205-211. 
McCarty JH (2005) Cell biology of the neurovascular unit: implications for drug delivery across the blood-brain barrier. Assay Drug Dev Technol 3:89-95.

McMahon G (2000) VEGF receptor signaling in tumor angiogenesis. Oncologist 5:3-10.

Meany HJ, Fox E, McCully C, Tucker C and Balis FM (2008) The plasma and cerebrospinal fluid pharmacokinetics of erlotinib and its active metabolite (OSI420) after intravenous administration of erlotinib in non-human primates. Cancer Chemother Pharmacol 62:387-392.

Mellinghoff IK, Wang MY, Vivanco I, Haas-Kogan DA, Zhu S, Dia EQ, Lu KV, Yoshimoto K, Huang JH, Chute DJ, Riggs BL, Horvath S, Liau LM, Cavenee WK, Rao PN, Beroukhim R, Peck TC, Lee JC, Sellers WR, Stokoe D, Prados M, Cloughesy TF, Sawyers CL and Mischel PS (2005) Molecular determinants of the response of glioblastomas to EGFR kinase inhibitors. N Engl J Med 353:20122024.

Menacherry S, Hubert W and Justice JB, Jr. (1992) In vivo calibration of microdialysis probes for exogenous compounds. Anal Chem 64:577-583.

Mercer RW, Tyler MA, Ulasov IV and Lesniak MS (2009) Targeted Therapies for Malignant Glioma Progress and Potential. BioDrugs 23:25-35.

Metzger D and Feil R (1999) Engineering the mouse genome by site-specific recombination. Current opinion in biotechnology 10:470-476.

Minniti G, De Sanctis V, Muni R, Filippone F, Bozzao A, Valeriani M, Osti MF, De Paula U, Lanzetta G, Tombolini V and Maurizi Enrici R (2008) Radiotherapy plus concomitant and adjuvant temozolomide for glioblastoma in elderly patients. $J$ Neurooncol 88:97-103.

Miyagami M and Katayama Y (2005) Angiogenesis of glioma: evaluation of ultrastructural characteristics of microvessels and tubular bodies (Weibel-Palade) in endothelial cells and immunohistochemical findings with VEGF and p53 protein. Med Mol Morphol 38:36-42.

Mohri M, Nitta H and Yamashita J (2000) Expression of multidrug resistance-associated protein (MRP) in human gliomas. $J$ Neurooncol 49:105-115.

Mori S, Ohtsuki S, Takanaga H, Kikkawa T, Kang YS and Terasaki T (2004) Organic anion transporter 3 is involved in the brain-to-blood efflux transport of thiopurine nucleobase analogs. $J$ Neurochem 90:931-941. 
Mori S, Takanaga H, Ohtsuki S, Deguchi T, Kang YS, Hosoya K and Terasaki T (2003) Rat organic anion transporter 3 (rOAT3) is responsible for brain-to-blood efflux of homovanillic acid at the abluminal membrane of brain capillary endothelial cells. J Cereb Blood Flow Metab 23:432-440.

Morikawa S, Baluk P, Kaidoh T, Haskell A, Jain RK and McDonald DM (2002) Abnormalities in pericytes on blood vessels and endothelial sprouts in tumors. The American journal of pathology 160:985-1000.

Motl S, Zhuang Y, Waters CM and Stewart CF (2006) Pharmacokinetic considerations in the treatment of CNS tumours. Clin Pharmacokinet 45:871-903.

Motohashi H, Sakurai Y, Saito H, Masuda S, Urakami Y, Goto M, Fukatsu A, Ogawa O and Inui K (2002) Gene expression levels and immunolocalization of organic ion transporters in the human kidney. J Am Soc Nephrol 13:866-874.

Moyer JD, Barbacci EG, Iwata KK, Arnold L, Boman B, Cunningham A, DiOrio C, Doty J, Morin MJ, Moyer MP, Neveu M, Pollack VA, Pustilnik LR, Reynolds MM, Sloan D, Theleman A and Miller P (1997) Induction of apoptosis and cell cycle arrest by CP-358,774, an inhibitor of epidermal growth factor receptor tyrosine kinase. Cancer Res 57:4838-4848.

Muldoon LL, Soussain C, Jahnke K, Johanson C, Siegal T, Smith QR, Hall WA, Hynynen K, Senter PD, Peereboom DM and Neuwelt EA (2007) Chemotherapy delivery issues in central nervous system malignancy: a reality check. J Clin Oncol 25:2295-2305.

Nabors MW, Griffin CA, Zehnbauer BA, Hruban RH, Phillips PC, Grossman SA, Brem $\mathrm{H}$ and Colvin OM (1991) Multidrug resistance gene (MDR1) expression in human brain tumors. JNeurosurg 75:941-946.

Nagata Y, Kusuhara H, Endou H and Sugiyama Y (2002) Expression and functional characterization of rat organic anion transporter 3 (rOat3) in the choroid plexus. Mol Pharmacol 61:982-988.

Neville K, Parise RA, Thompson P, Aleksic A, Egorin MJ, Balis FM, McGuffey L, McCully C, Berg SL and Blaney SM (2004) Plasma and cerebrospinal fluid pharmacokinetics of imatinib after administration to nonhuman primates. Clin Cancer Res 10:2525-2529.

Newcomb EW, Alonso M, Sung T and Miller DC (2000) Incidence of p14ARF gene deletion in high-grade adult and pediatric astrocytomas. Hum Pathol 31:115-119. 
Nezu J, Tamai I, Oku A, Ohashi R, Yabuuchi H, Hashimoto N, Nikaido H, Sai Y, Koizumi A, Shoji Y, Takada G, Matsuishi T, Yoshino M, Kato H, Ohura T, Tsujimoto G, Hayakawa J, Shimane M and Tsuji A (1999) Primary systemic carnitine deficiency is caused by mutations in a gene encoding sodium iondependent carnitine transporter. Nat Genet 21:91-94.

Nies AT, Jedlitschky G, Konig J, Herold-Mende C, Steiner HH, Schmitt HP and Keppler D (2004) Expression and immunolocalization of the multidrug resistance proteins, MRP1-MRP6 (ABCC1-ABCC6), in human brain. Neuroscience 129:349-360.

Nies AT, Koepsell H, Damme K and Schwab M (2011) Organic cation transporters (OCTs, MATEs), in vitro and in vivo evidence for the importance in drug therapy. Handbook of experimental pharmacology:105-167.

Nister M, Claesson-Welsh L, Eriksson A, Heldin CH and Westermark B (1991) Differential expression of platelet-derived growth factor receptors in human malignant glioma cell lines. J Biol Chem 266:16755-16763.

Nobmann S, Bauer B and Fricker G (2001) Ivermectin excretion by isolated functionally intact brain endothelial capillaries. Br J Pharmacol 132:722-728.

Noe B, Hagenbuch B, Stieger B and Meier PJ (1997) Isolation of a multispecific organic anion and cardiac glycoside transporter from rat brain. Proc Natl Acad Sci U S A 94:10346-10350.

Ohtsuki S, Kikkawa T, Mori S, Hori S, Takanaga H, Otagiri M and Terasaki T (2004) Mouse reduced in osteosclerosis transporter functions as an organic anion transporter 3 and is localized at abluminal membrane of blood-brain barrier. Journal of Pharmacology and Experimental Therapeutics 309:1273-1281.

Oravcova J, Bohs B and Lindner W (1996) Drug-protein binding sites. New trends in analytical and experimental methodology. J Chromatogr B Biomed Appl 677:128.

Ose A, Kusuhara H, Endo C, Tohyama K, Miyajima M, Kitamura S and Sugiyama Y (2010) Functional characterization of mouse organic anion transporting peptide 1a4 in the uptake and efflux of drugs across the blood-brain barrier. Drug Metab Dispos 38:168-176.

Padera TP, Kadambi A, di Tomaso E, Carreira CM, Brown EB, Boucher Y, Choi NC, Mathisen D, Wain J, Mark EJ, Munn LL and Jain RK (2002) Lymphatic metastasis in the absence of functional intratumor lymphatics. Science 296:18831886.

Padera TP, Stoll BR, Tooredman JB, Capen D, di Tomaso E and Jain RK (2004) Pathology: cancer cells compress intratumour vessels. Nature 427:695. 
Pan YF, Feng J, Cheng QY and Li FZ (2007) Intracerebral microdialysis technique and its application on brain pharmacokinetic-pharmacodynamic study. Arch Pharm Res 30:1635-1645.

Pandita A, Aldape KD, Zadeh G, Guha A and James CD (2004) Contrasting in vivo and in vitro fates of glioblastoma cell subpopulations with amplified EGFR. Genes Chromosomes Cancer 39:29-36.

Papadopoulos MC, Saadoun S, Binder DK, Manley GT, Krishna S and Verkman AS (2004) Molecular mechanisms of brain tumor edema. Neuroscience 129:10111020.

Pardridge WM (1998) CNS drug design based on principles of blood-brain barrier transport. J Neurochem 70:1781-1792.

Pardridge WM (1999) Blood-brain barrier biology and methodology. J Neurovirol 5:556569.

Pardridge WM (2005) The blood-brain barrier: bottleneck in brain drug development. NeuroRx 2:3-14.

Parsons DW, Jones S, Zhang X, Lin JC, Leary RJ, Angenendt P, Mankoo P, Carter H, Siu IM, Gallia GL, Olivi A, McLendon R, Rasheed BA, Keir S, Nikolskaya T, Nikolsky Y, Busam DA, Tekleab H, Diaz LA, Jr., Hartigan J, Smith DR, Strausberg RL, Marie SK, Shinjo SM, Yan H, Riggins GJ, Bigner DD, Karchin R, Papadopoulos N, Parmigiani G, Vogelstein B, Velculescu VE and Kinzler KW (2008) An integrated genomic analysis of human glioblastoma multiforme.

Science 321:1807-1812.

Paugh BS, Qu C, Jones C, Liu Z, Adamowicz-Brice M, Zhang J, Bax DA, Coyle B, Barrow J, Hargrave D, Lowe J, Gajjar A, Zhao W, Broniscer A, Ellison DW, Grundy RG and Baker SJ (2010a) Integrated molecular genetic profiling of pediatric high-grade gliomas reveals key differences with the adult disease. J Clin Oncol 28:3061-3068.

Paugh BS, Qu CX, Zhang JY, Geyer JR, Chi SS, da Silva NS, Baker J, Gajjar A, Ellison DW, Broniscer A and Baker SJ (2010b) Genome-Wide Analyses of Diffuse Intrinsic Pontine Gliomas Reveal Key Differences with Supratentorial Pediatric Glioblastomas and Adult Glioblastomas. Neuro-Oncology 12:85-85.

Paxinos G and Franklin KBJ (2001) The mouse brain in stereotaxic coordinates, Academic Press, San Diego. 
Peereboom DM, Shepard DR, Ahluwalia MS, Brewer CJ, Agarwal N, Stevens GH, Suh JH, Toms SA, Vogelbaum MA, Weil RJ, Elson P and Barnett GH (2010) Phase II trial of erlotinib with temozolomide and radiation in patients with newly diagnosed glioblastoma multiforme. J Neurooncol 98:93-99.

Phillips HS, Kharbanda S, Chen R, Forrest WF, Soriano RH, Wu TD, Misra A, Nigro JM, Colman H, Soroceanu L, Williams PM, Modrusan Z, Feuerstein BG and Aldape K (2006) Molecular subclasses of high-grade glioma predict prognosis, delineate a pattern of disease progression, and resemble stages in neurogenesis. Cancer Cell 9:157-173.

Phuphanich S, Edwards MS, Levin VA, Vestnys PS, Wara WM, Davis RL and Wilson CB (1984) Supratentorial malignant gliomas of childhood. Results of treatment with radiation therapy and chemotherapy. J Neurosurg 60:495-499.

Pietras K, Sjoblom T, Rubin K, Heldin CH and Ostman A (2003) PDGF receptors as cancer drug targets. Cancer Cell 3:439-443.

Pitz MW, Desai A, Grossman SA and Blakeley JO (2011) Tissue concentration of systemically administered antineoplastic agents in human brain tumors. $J$ Neurooncol 104:629-638.

PL G and GM P (2003) Blood-brain barrier efflux transport. J Pharm Sci 92:1739-1753.

Plate KH, Breier G, Weich HA, Mennel HD and Risau W (1994) Vascular endothelial growth factor and glioma angiogenesis: coordinate induction of VEGF receptors, distribution of VEGF protein and possible in vivo regulatory mechanisms. Int $J$ Cancer 59:520-529.

Plate KH, Breier G, Weich HA and Risau W (1992) Vascular endothelial growth factor is a potential tumour angiogenesis factor in human gliomas in vivo. Nature 359:845848.

Pluck A (1996) Conditional mutagenesis in mice: the Cre/loxP recombination system. Int J Exp Pathol 77:269-278.

Pollack IF, Finkelstein SD, Burnham J, Holmes EJ, Hamilton RL, Yates AJ, Finlay JL and Sposto R (2001) Age and TP53 mutation frequency in childhood malignant gliomas: results in a multi-institutional cohort. Cancer Res 61:7404-7407.

Pollack IF, Hamilton RL, Burger PC, Brat DJ, Rosenblum MK, Murdoch GH, Nikiforova MN, Holmes EJ, Zhou T, Cohen KJ and Jakacki RI (2010) Akt activation is a common event in pediatric malignant gliomas and a potential adverse prognostic marker: a report from the Children's Oncology Group. J Neurooncol 99:155-163. 
Pollack IF, Hamilton RL, James CD, Finkelstein SD, Burnham J, Yates AJ, Holmes EJ, Zhou T and Finlay JL (2006) Rarity of PTEN deletions and EGFR amplification in malignant gliomas of childhood: results from the Children's Cancer Group 945 cohort. J Neurosurg 105:418-424.

Poller B, Iusuf D, Sparidans RW, Wagenaar E, Beijnen JH and Schinkel AH (2011) Differential impact of P-glycoprotein (ABCB1) and breast cancer resistance protein (ABCG2) on axitinib brain accumulation and oral plasma pharmacokinetics. Drug Metab Dispos 39:729-735.

Polli JW, Olson KL, Chism JP, St John-Williams LA, Yeager RL, Woodard SM, Otto VR, Castellino S and Demby VE (2008) An Unexpected Synergist Role of Pglycoprotein and Breast Cancer Resistance Protein on the CNS Penetration of the Tyrosine Kinase Inhibitor Lapatinib (GW572016). Drug Metab Dispos 37:439442.

Porter KR, McCarthy BJ, Freels S, Kim Y and Davis FG (2010) Prevalence estimates for primary brain tumors in the United States by age, gender, behavior, and histology. Neuro Oncol 12:520-527.

Prados MD, Chang SM, Butowski N, DeBoer R, Parvataneni R, Carliner H, Kabuubi P, Ayers-Ringler J, Rabbitt J, Page M, Fedoroff A, Sneed PK, Berger MS, McDermott MW, Parsa AT, Vandenberg S, James CD, Lamborn KR, Stokoe D and Haas-Kogan DA (2009) Phase II study of erlotinib plus temozolomide during and after radiation therapy in patients with newly diagnosed glioblastoma multiforme or gliosarcoma. J Clin Oncol 27:579-584.

Prados MD, Lamborn KR, Chang S, Burton E, Butowski N, Malec M, Kapadia A, Rabbitt J, Page MS, Fedoroff A, Xie D and Kelley SK (2006) Phase 1 study of erlotinib $\mathrm{HCl}$ alone and combined with temozolomide in patients with stable or recurrent malignant glioma. Neuro Oncol 8:67-78.

Radaelli E, Ceruti R, Patton V, Russo M, Degrassi A, Croci V, Caprera F, Stortini G, Scanziani E, Pesenti E and Alzani R (2009) Immunohistopathological and neuroimaging characterization of murine orthotopic xenograft models of glioblastoma multiforme recapitulating the most salient features of human disease. Histology and Histopathology 24:879-891.

Raizer JJ, Abrey LE, Lassman AB, Chang SM, Lamborn KR, Kuhn JG, Yung WK, Gilbert MR, Aldape KA, Wen PY, Fine HA, Mehta M, Deangelis LM, Lieberman F, Cloughesy TF, Robins HI, Dancey J and Prados MD (2010a) A phase II trial of erlotinib in patients with recurrent malignant gliomas and nonprogressive glioblastoma multiforme postradiation therapy. Neuro Oncol 12:95-103. 
Raizer JJ, Abrey LE, Lassman AB, Chang SM, Lamborn KR, Kuhn JG, Yung WK, Gilbert MR, Aldape KD, Wen PY, Fine HA, Mehta M, Deangelis LM, Lieberman F, Cloughesy TF, Robins HI, Dancey J and Prados MD (2010b) A phase I trial of erlotinib in patients with nonprogressive glioblastoma multiforme postradiation therapy, and recurrent malignant gliomas and meningiomas. Neuro Oncol 12:8794.

Rao VV, Dahlheimer JL, Bardgett ME, Snyder AZ, Finch RA, Sartorelli AC and Piwnica-Worms D (1999a) Choroid plexus epithelial expression of MDR1 P glycoprotein and multidrug resistance-associated protein contribute to the bloodcerebrospinal-fluid drug-permeability barrier. ProcNatlAcadSciUSA 96:39003905.

Rao VV, Dahlheimer JL, Bardgett ME, Snyder AZ, Finch RA, Sartorelli AC and Piwnica-Worms D (1999b) Choroid plexus epithelial expression of MDR1 P glycoprotein and multidrug resistance-associated protein contribute to the bloodcerebrospinal-fluid drug-permeability barrier. Proc Natl Acad Sci U S A 96:39003905.

Raymond E, Brandes AA, Dittrich C, Fumoleau P, Coudert B, Clement PM, Frenay M, Rampling R, Stupp R, Kros JM, Heinrich MC, Gorlia T, Lacombe D and van den Bent MJ (2008) Phase II study of imatinib in patients with recurrent gliomas of various histologies: a European Organisation for Research and Treatment of Cancer Brain Tumor Group Study. J Clin Oncol 26:4659-4665.

Raymond E, Faivre S and Armand JP (2000) Epidermal growth factor receptor tyrosine kinase as a target for anticancer therapy. Drugs 60:15-23.

Reardon DA, Quinn JA, Vredenburgh JJ, Gururangan S, Friedman AH, Desjardins A, Sathornsumetee S, Herndon JE, 2nd, Dowell JM, McLendon RE, Provenzale JM, Sampson JH, Smith RP, Swaisland AJ, Ochs JS, Lyons P, Tourt-Uhlig S, Bigner DD, Friedman HS and Rich JN (2006) Phase 1 trial of gefitinib plus sirolimus in adults with recurrent malignant glioma. Clin Cancer Res 12:860-868.

Reardon DA, Turner S, Peters KB, Desjardins A, Gururangan S, Sampson JH, McLendon RE, Herndon JE, 2nd, Jones LW, Kirkpatrick JP, Friedman AH, Vredenburgh JJ, Bigner DD and Friedman HS (2011) A review of VEGF/VEGFR-targeted therapeutics for recurrent glioblastoma. J Natl Compr Canc Netw 9:414-427.

Redzic ZB and Segal MB (2004) The structure of the choroid plexus and the physiology of the choroid plexus epithelium. Adv Drug Deliv Rev 56:1695-1716.

Reichel A (2009) Addressing central nervous system (CNS) penetration in drug discovery: basics and implications of the evolving new concept. Chem Biodivers 6:2030-2049. 
Reilly KM, Loisel DA, Bronson RT, McLaughlin ME and Jacks T (2000) Nf1;Trp53 mutant mice develop glioblastoma with evidence of strain-specific effects. Nat Genet 26:109-113.

Ren H, Yang BF and Rainov NG (2007) Receptor tyrosine kinases as therapeutic targets in malignant glioma. Rev Recent Clin Trials 2:87-101.

Rennick BR (1981) Renal tubule transport of organic cations. Am J Physiol 240:F83-89.

Rich JN, Reardon DA, Peery T, Dowell JM, Quinn JA, Penne KL, Wikstrand CJ, Van Duyn LB, Dancey JE, McLendon RE, Kao JC, Stenzel TT, hmed Rasheed BK, Tourt-Uhlig SE, Herndon JE, Vredenburgh JJ, Sampson JH, Friedman AH, Bigner DD and Friedman HS (2004) Phase II trial of gefitinib in recurrent glioblastoma. Journal of Clinical Oncology 22:133-142.

Richmond A and Su Y (2008) Mouse xenograft models vs GEM models for human cancer therapeutics. Dis Model Mech 1:78-82.

Richmond CR (1985) Icrp Report of the Task Group on Reference Man. Int J Nucl Med Biol 12:251-251.

Roberts LM, Black DS, Raman C, Woodford K, Zhou M, Haggerty JE, Yan AT, Cwirla SE and Grindstaff KK (2008) Subcellular localization of transporters along the rat blood-brain barrier and blood-cerebral-spinal fluid barrier by in vivo biotinylation. Neuroscience 155:423-438.

Robinson DR, Wu YM and Lin SF (2000) The protein tyrosine kinase family of the human genome. Oncogene 19:5548-5557.

Roe M, Folkes A, Ashworth P, Brumwell J, Chima L, Hunjan S, Pretswell I, Dangerfield W, Ryder H and Charlton P (1999) Reversal of P-glycoprotein mediated multidrug resistance by novel anthranilamide derivatives. Bioorg Med Chem Lett 9:595-600.

Rong Y, Durden DL, Van Meir EG and Brat DJ (2006) 'Pseudopalisading' necrosis in glioblastoma: a familiar morphologic feature that links vascular pathology, hypoxia, and angiogenesis. J Neuropathol Exp Neurol 65:529-539.

Rubin LL and Staddon JM (1999) The cell biology of the blood-brain barrier. Annu Rev Neurosci 22:11-28.

Rudin CM, Liu W, Desai A, Karrison T, Jiang X, Janisch L, Das S, Ramirez J, Poonkuzhali B, Schuetz E, Fackenthal DL, Chen P, Armstrong DK, Brahmer JR, Fleming GF, Vokes EE, Carducci MA and Ratain MJ (2008) Pharmacogenomic and pharmacokinetic determinants of erlotinib toxicity. J Clin Oncol 26:11191127. 
Rutten EH, Kazem I, Slooff JL and Walder AH (1981) Post operative radiation therapy in the management of brain astrocytomata-retrospective study of 142 patients. Int $J$ Radiat Oncol Biol Phys 7:191-195.

Saleh M, Stacker SA and Wilks AF (1996) Inhibition of growth of C6 glioma cells in vivo by expression of antisense vascular endothelial growth factor sequence. Cancer Res 56:393-401.

Sarkaria JN, Yang L, Grogan PT, Kitange GJ, Carlson BL, Schroeder MA, Galanis E, Giannini C, Wu W, Dinca EB and James CD (2007) Identification of molecular characteristics correlated with glioblastoma sensitivity to EGFR kinase inhibition through use of an intracranial xenograft test panel. Mol Cancer Ther 6:1167-1174.

Schinkel AH, Smit JJ, van Tellingen O, Beijnen JH, Wagenaar E, van Deemter L, Mol CA, van der Valk MA, Robanus-Maandag EC and te Riele HP (1994) Disruption of the mouse mdrla P-glycoprotein gene leads to a deficiency in the blood-brain barrier and to increased sensitivity to drugs. Cell 77:491-502.

Schinkel AH, Wagenaar E, Mol CA and van Deemter L (1996) P-glycoprotein in the blood-brain barrier of mice influences the brain penetration and pharmacological activity of many drugs. $J$ Clin Invest 97:2517-2524.

Schinkel AH, Wagenaar E, van Deemter L, Mol CA and Borst P (1995) Absence of the mdr1a P-Glycoprotein in mice affects tissue distribution and pharmacokinetics of dexamethasone, digoxin, and cyclosporin A. J Clin Invest 96:1698-1705.

Schmidt NO, Westphal M, Hagel C, Ergun S, Stavrou D, Rosen EM and Lamszus K (1999) Levels of vascular endothelial growth factor, hepatocyte growth factor/scatter factor and basic fibroblast growth factor in human gliomas and their relation to angiogenesis. Int J Cancer 84:10-18.

Schulte A, Gunther HS, Martens T, Zapf S, Riethdorf S, Wulfing C, Stoupiec M, Westphal M and Lamszus K (2012) Glioblastoma Stem-like Cell Lines with Either Maintenance or Loss of High-Level EGFR Amplification, Generated via Modulation of Ligand Concentration. Clin Cancer Res 18:1901-1913.

Schumann GB and Crisman LG (1985) Cerebrospinal fluid cytopathology. Clin Lab Med 5:275-302.

Sharma PS, Sharma R and Tyagi T (2011) VEGF/VEGFR pathway inhibitors as antiangiogenic agents: present and future. Curr Cancer Drug Targets 11:624-653.

Shen DD, Artru AA and Adkison KK (2004) Principles and applicability of CSF sampling for the assessment of CNS drug delivery and pharmacodynamics. $A d v$ Drug Deliv Rev 56:1825-1857. 
Shen J, Carcaboso AM, Hubbard KE, Tagen M, Wynn HG, Panetta JC, Waters CM, Elmeliegy MA and Stewart CF (2009) Compartment-specific roles of ATPbinding cassette transporters define differential topotecan distribution in brain parenchyma and cerebrospinal fluid. Cancer Res 69:5885-5892.

Shepherd FA, Rodrigues Pereira J, Ciuleanu T, Tan EH, Hirsh V, Thongprasert S, Campos D, Maoleekoonpiroj S, Smylie M, Martins R, van Kooten M, Dediu M, Findlay B, Tu D, Johnston D, Bezjak A, Clark G, Santabarbara P and Seymour L (2005) Erlotinib in previously treated non-small-cell lung cancer. $N$ Engl J Med 353:123-132.

Sikic BI, Fisher GA, Lum BL, Halsey J, Beketic-Oreskovic L and Chen G (1997) Modulation and prevention of multidrug resistance by inhibitors of P-glycoprotein. Cancer Chemother Pharmacol 40:13-19.

Slitt AL, Cherrington NJ, Hartley DP, Leazer TM and Klaassen CD (2002) Tissue distribution and renal developmental changes in rat organic cation transporter mRNA levels. Drug Metab Dispos 30:212-219.

Smith DA, Di L and Kerns EH (2010) The effect of plasma protein binding on in vivo efficacy: misconceptions in drug discovery. Nature Reviews Drug Discovery 9:929-939.

Smith MA, Freidlin B, Ries LA and Simon R (1998) Trends in reported incidence of primary malignant brain tumors in children in the United States. J Natl Cancer Inst 90:1269-1277.

Song KS, Phi JH, Cho BK, Wang KC, Lee JY, Kim DG, Kim IH, Ahn HS, Park SH and Kim SK (2010) Long-term outcomes in children with glioblastoma. J Neurosurg Pediatr 6:145-149.

Squatrito M and Holland EC (2011) DNA damage response and growth factor signaling pathways in gliomagenesis and therapeutic resistance. Cancer Res 71:5945-5949.

Stain-Texier F, Boschi G, Sandouk P and Scherrmann JM (1999) Elevated concentrations of morphine 6-beta-D-glucuronide in brain extracellular fluid despite low bloodbrain barrier permeability. Br J Pharmacol 128:917-924.

Stenken JA, Chen R and Yuan XN (2001) Influence of geometry and equilibrium chemistry on relative recovery during enhanced microdialysis. Analytica Chimica Acta 436:21-29.

Stommel JM, Kimmelman AC, Ying H, Nabioullin R, Ponugoti AH, Wiedemeyer R, Stegh AH, Bradner JE, Ligon KL, Brennan C, Chin L and DePinho RA (2007) Coactivation of receptor tyrosine kinases affects the response of tumor cells to targeted therapies. Science 318:287-290. 
Stupp R, Hegi ME, Gilbert MR and Chakravarti A (2007) Chemoradiotherapy in malignant glioma: standard of care and future directions. J Clin Oncol 25:41274136.

Stupp R, Mason WP, van den Bent MJ, Weller M, Fisher B, Taphoorn MJ, Belanger K, Brandes AA, Marosi C, Bogdahn U, Curschmann J, Janzer RC, Ludwin SK, Gorlia T, Allgeier A, Lacombe D, Cairncross JG, Eisenhauer E and Mirimanoff RO (2005) Radiotherapy plus concomitant and adjuvant temozolomide for glioblastoma. N Engl J Med 352:987-996.

Sun L and Stenken JA (2003) Improving microdialysis extraction efficiency of lipophilic eicosanoids. J Pharm Biomed Anal 33:1059-1071.

Sun M, Behrens C, Feng L, Ozburn N, Tang X, Yin G, Komaki R, Varella-Garcia M, Hong WK, Aldape KD and Wistuba, II (2009) HER family receptor abnormalities in lung cancer brain metastases and corresponding primary tumors. Clin Cancer Res 15:4829-4837.

Sung T, Miller DC, Hayes RL, Alonso M, Yee H and Newcomb EW (2000) Preferential inactivation of the p53 tumor suppressor pathway and lack of EGFR amplification distinguish de novo high grade pediatric astrocytomas from de novo adult astrocytomas. Brain Pathol 10:249-259.

Suri V, Das P, Pathak P, Jain A, Sharma MC, Borkar SA, Suri A, Gupta D and Sarkar C (2009) Pediatric glioblastomas: a histopathological and molecular genetic study. Neuro Oncol 11:274-280.

Sykes D, Sweet DH, Lowes S, Nigam SK, Pritchard JB and Miller DS (2004) Organic anion transport in choroid plexus from wild-type and organic anion transporter 3 (Slc22a8)-null mice. Am J Physiol Renal Physiol 286:F972-978.

Szentirmai O, Baker CH, Lin N, Szucs S, Takahashi M, Kiryu S, Kung AL, Mulligan RC and Carter BS (2006) Noninvasive bioluminescence imaging of luciferase expressing intracranial U87 xenografts: correlation with magnetic resonance imaging determined tumor volume and longitudinal use in assessing tumor growth and antiangiogenic treatment effect. Neurosurgery 58:365-372.

Tahara H, Kusuhara H, Maeda K, Koepsell H, Fuse E and Sugiyama Y (2006) Inhibition of oat3-mediated renal uptake as a mechanism for drug-drug interaction between fexofenadine and probenecid. Drug Metab Dispos 34:743-747.

TCGA (2008) Comprehensive genomic characterization defines human glioblastoma genes and core pathways. Nature 455:1061-1068.

Terasaki T and Hosoya K (1999) The blood-brain barrier efflux transporters as a detoxifying system for the brain. AdvDrug DelivRev 36:195-209. 
Terasaki T and Ohtsuki S (2005) Brain-to-blood transporters for endogenous substrates and xenobiotics at the blood-brain barrier: an overview of biology and methodology. NeuroRx 2:63-72.

Thaker NG and Pollack IF (2009) Molecularly targeted therapies for malignant glioma: rationale for combinatorial strategies. Expert Rev Neurother 9:1815-1836.

Thiebaut F, Tsuruo T, Hamada H, Gottesman MM, Pastan I and Willingham MC (1989) Immunohistochemical localization in normal tissues of different epitopes in the multidrug transport protein P170: evidence for localization in brain capillaries and crossreactivity of one antibody with a muscle protein. $J$ Histochem Cytochem 37:159-164.

Thorarinsdottir HK, Santi M, McCarter R, Rushing EJ, Cornelison R, Jales A and MacDonald TJ (2008) Protein expression of platelet-derived growth factor receptor correlates with malignant histology and PTEN with survival in childhood gliomas. Clin Cancer Res 14:3386-3394.

Togashi Y, Masago K, Fukudo M, Terada T, Fujita S, Irisa K, Sakamori Y, Kim YH, Mio $\mathrm{T}$, Inui $\mathrm{K}$ and Mishima $\mathrm{M}$ Cerebrospinal fluid concentration of erlotinib and its active metabolite OSI-420 in patients with central nervous system metastases of non-small cell lung cancer. $J$ Thorac Oncol 5:950-955.

Tredan O, Galmarini CM, Patel K and Tannock IF (2007) Drug resistance and the solid tumor microenvironment. $J$ Natl Cancer Inst 99:1441-1454.

Tsai TH (2003) Assaying protein unbound drugs using microdialysis techniques. $J$ Chromatogr B Analyt Technol Biomed Life Sci 797:161-173.

Ucuzian AA, Gassman AA, East AT and Greisler HP (2010) Molecular mediators of angiogenesis. Journal of burn care \& research : official publication of the American Burn Association 31:158-175.

Urquhart BL and Kim RB (2009) Blood-brain barrier transporters and response to CNSactive drugs. Eur J Clin Pharmacol 65:1063-1070.

Valles F, Fiandaca MS, Bringas J, Dickinson P, LeCouteur R, Higgins R, Berger M, Forsayeth J and Bankiewicz KS (2009) Anatomic compression caused by highvolume convection-enhanced delivery to the brain. Neurosurgery 65:579-585.

van den Bent MJ, Brandes AA, Rampling R, Kouwenhoven MC, Kros JM, Carpentier AF, Clement PM, Frenay M, Campone M, Baurain JF, Armand JP, Taphoorn MJ, Tosoni A, Kletzl H, Klughammer B, Lacombe D and Gorlia T (2009a) Randomized phase II trial of erlotinib versus temozolomide or carmustine in recurrent glioblastoma: EORTC brain tumor group study 26034. J Clin Oncol 27:1268-1274. 
van den Bent MJ, Vogelbaum MA, Wen PY, Macdonald DR and Chang SM (2009b) End point assessment in gliomas: novel treatments limit usefulness of classical Macdonald's Criteria. J Clin Oncol 27:2905-2908.

Vanbree JBMM, Baljet AV, Vangeyt A, Deboer AG, Danhof M and Breimer DD (1989) The Unit Impulse-Response Procedure for the Pharmacokinetic Evaluation of Drug Entry into the Central Nervous-System. Journal of Pharmacokinetics and Biopharmaceutics 17:441-462.

Verhaak RG, Hoadley KA, Purdom E, Wang V, Qi Y, Wilkerson MD, Miller CR, Ding L, Golub T, Mesirov JP, Alexe G, Lawrence M, O'Kelly M, Tamayo P, Weir BA, Gabriel S, Winckler W, Gupta S, Jakkula L, Feiler HS, Hodgson JG, James CD, Sarkaria JN, Brennan C, Kahn A, Spellman PT, Wilson RK, Speed TP, Gray JW, Meyerson M, Getz G, Perou CM and Hayes DN (2010) Integrated genomic analysis identifies clinically relevant subtypes of glioblastoma characterized by abnormalities in PDGFRA, IDH1, EGFR, and NF1. Cancer Cell 17:98-110.

Vistelle R, Jaussaud R, Trenque T and Wiczewski M (1994) Rapid and simple cannulation technique for repeated sampling of cerebrospinal fluid in the conscious rabbit. Lab Anim Sci 44:362-364.

Vogelbaum MA, Agarwal S, Manchanda P, Ohlfest JR and Elmquist WF (2011) Inhibition of Active Efflux at the Bbb Improves Delivery and Efficacy of Erlotinib in an Orthotopic Xenograft Model of Glioma. Neuro-Oncology 13:118119.

Vredenburgh JJ, Desjardins A, Herndon JE, 2nd, Marcello J, Reardon DA, Quinn JA, Rich JN, Sathornsumetee S, Gururangan S, Sampson J, Wagner M, Bailey L, Bigner DD, Friedman AH and Friedman HS (2007) Bevacizumab plus irinotecan in recurrent glioblastoma multiforme. J Clin Oncol 25:4722-4729.

Wang F, Zhou F, Kruh GD and Gallo JM (2010) Influence of blood-brain barrier efflux pumps on the distribution of vincristine in brain and brain tumors. Neuro Oncol 12:1043-1049.

Wang S, Guo P, Wang X, Zhou Q and Gallo JM (2008) Preclinical pharmacokinetic/pharmacodynamic models of gefitinib and the design of equivalent dosing regimens in EGFR wild-type and mutant tumor models. Molecular cancer therapeutics 7:407-417.

Wang Y, Wong SL and Sawchuk RJ (1993a) Microdialysis calibration using retrodialysis and zero-net flux: application to a study of the distribution of zidovudine to rabbit cerebrospinal fluid and thalamus. Pharm Res 10:1411-1419. 
Wang Y, Yang J, Zheng H, Tomasek GJ, Zhang P, McKeever PE, Lee EY and Zhu Y (2009) Expression of mutant p53 proteins implicates a lineage relationship between neural stem cells and malignant astrocytic glioma in a murine model. Cancer Cell 15:514-526.

Wang YF, Wong SL and Sawchuk RJ (1993b) Microdialysis Calibration Using Retrodialysis and Zero-Net Flux - Application to a Study of the Distribution of Zidovudine to Rabbit Cerebrospinal-Fluid and Thalamus. Pharmaceutical Research 10:1411-1419.

Wen PY and Kesari S (2008) Malignant gliomas in adults. N Engl J Med 359:492-507.

Wen PY, Yung WK, Lamborn KR, Dahia PL, Wang Y, Peng B, Abrey LE, Raizer J, Cloughesy TF, Fink K, Gilbert M, Chang S, Junck L, Schiff D, Lieberman F, Fine HA, Mehta M, Robins HI, DeAngelis LM, Groves MD, Puduvalli VK, Levin V, Conrad C, Maher EA, Aldape K, Hayes M, Letvak L, Egorin MJ, Capdeville R, Kaplan R, Murgo AJ, Stiles C and Prados MD (2006) Phase I/II study of imatinib mesylate for recurrent malignant gliomas: North American Brain Tumor Consortium Study 99-08. Clin Cancer Res 12:4899-4907.

Westerhout J, Danhof M and De Lange EC (2011) Preclinical prediction of human brain target site concentrations: considerations in extrapolating to the clinical setting. $J$ Pharm Sci 100:3577-3593.

Wick W, Weller M, van den Bent M and Stupp R (2010) Bevacizumab and recurrent malignant gliomas: a European perspective. J Clin Oncol 28:188-189.

Wijnholds J, deLange EC, Scheffer GL, van den Berg DJ, Mol CA, van der Valk M, Schinkel AH, Scheper RJ, Breimer DD and Borst P (2000) Multidrug resistance protein 1 protects the choroid plexus epithelium and contributes to the bloodcerebrospinal fluid barrier. J Clin Invest 105:279-285.

Wijnholds J, Evers R, van Leusden MR, Mol CA, Zaman GJ, Mayer U, Beijnen JH, van der Valk M, Krimpenfort P and Borst P (1997) Increased sensitivity to anticancer drugs and decreased inflammatory response in mice lacking the multidrug resistance-associated protein. Nat Med 3:1275-1279.

WM P (2003) Blood-brain barrier genomics and the use of endogenous transporters to cause drug penetration into the brain. Curr Opin Drug Discov Devel 6:683-691.

Wolburg H and Lippoldt A (2002) Tight junctions of the blood-brain barrier: development, composition and regulation. Vascul Pharmacol 38:323-337.

Xiao A, Wu H, Pandolfi PP, Louis DN and Van Dyke T (2002) Astrocyte inactivation of the $\mathrm{pRb}$ pathway predisposes mice to malignant astrocytoma development that is accelerated by PTEN mutation. Cancer Cell 1:157-168. 
Zhang W, Mojsilovic-Petrovic J, Andrade MF, Zhang H, Ball M and Stanimirovic DB (2003) The expression and functional characterization of ABCG2 in brain endothelial cells and vessels. FASEB J 17:2085-2087.

Zhang Y, Han H, Elmquist WF and Miller DW (2000) Expression of various multidrug resistance-associated protein (MRP) homologues in brain microvessel endothelial cells. Brain Res 876:148-153.

Zhao M, He P, Rudek MA, Hidalgo M and Baker SD (2003) Specific method for determination of OSI-774 and its metabolite OSI-420 in human plasma by using liquid chromatography-tandem mass spectrometry. J Chromatogr B Analyt Technol Biomed Life Sci 793:413-420.

Zheng W, Aschner M and Ghersi-Egea JF (2003) Brain barrier systems: a new frontier in metal neurotoxicological research. Toxicol Appl Pharmacol 192:1-11.

Zhou Q and Gallo JM (2009) Differential effect of sunitinib on the distribution of temozolomide in an orthotopic glioma model. Neuro Oncol 11:301-310.

Zhu Y, Guignard F, Zhao D, Liu L, Burns DK, Mason RP, Messing A and Parada LF (2005) Early inactivation of p53 tumor suppressor gene cooperating with NF1 loss induces malignant astrocytoma. Cancer Cell 8:119-130.

Zhuang Y, Fraga CH, Hubbard KE, Hagedorn N, Panetta JC, Waters CM and Stewart CF (2006) Topotecan central nervous system penetration is altered by a tyrosine kinase inhibitor. Cancer Res 66:11305-11313. 


\section{VITA}

Mohamed Elmeliegy was born in Cairo, Egypt in 1981. He received a Bachelor of pharmaceutical sciences from Ain-Shams University in Cairo, Egypt in 2004. In 2007, he was accepted to join the Integrated Program in Biomedical Sciences (IPBS) in the University of Tennessee Health Science Center in Memphis in order to pursue his Ph.D. In 2011, Mohamed was awarded the Alma and Hal Reagan Fellowship by University of Tennessee for the year 2011-2012 for his work in the field of cancer research. His research advisor was Dr. Clinton Stewart, who served as a mentor and counselor providing knowledge, guidance and expertise through this successful dissertation process. In 2012, Mohamed has been awarded a combined post-doctoral fellowship from the University at Buffalo, New York and Novartis pharmaceuticals in East Hanover, New Jersey. 